Florida International University FIU Digital Commons

$11-9-2016$

\title{
A Comprehensive Decision Support Framework in the Front-End Phase of Major Transportation Projects
}

Nahid Vesali Mahmoud

Florida International University, nvesa001@fiu.edu

DOI: 10.25148 /etd.FIDC001262

Follow this and additional works at: https://digitalcommons.fiu.edu/etd

Part of the Construction Engineering and Management Commons

\section{Recommended Citation}

Vesali Mahmoud, Nahid, "A Comprehensive Decision Support Framework in the Front-End Phase of Major Transportation Projects" (2016). FIU Electronic Theses and Dissertations. 2723.

https://digitalcommons.fiu.edu/etd/2723 


\section{FLORIDA INTERNATIONAL UNIVERSITY}

Miami, Florida

\section{A COMPREHENSIVE DECISION SUPPORT FRAMEWORK}

IN THE FRONT-END PHASE OF MAJOR TRANSPORTATION PROJECTS

A dissertation submitted in partial fulfillment of

the requirements for the degree of

DOCTOR OF PHILOSOPHY

in

CIVIL ENGINEERING

by

Nahid Vesali Mahmoud 
To: $\quad$ Interim Dean Ranu Jung

College of Engineering and Computing

This dissertation, written by Nahid Vesali Mahmoud, and entitled A Comprehensive Decision Support Framework in the Front-End Phase of Major Transportation Projects, having been approved in respect to style and intellectual content, is referred to you for judgment.

We have read this dissertation and recommend that it be approved.

Young H. Kwak

Arindam G. Chowdhury

Berrin Tansel

Xia Jin, Co-Major Professor

M. Emre Bayraktar, Co-Major Professor

Date of Defense: November 9, 2016

The dissertation of Nahid Vesali Mahmoud is approved.

Interim Dean Ranu Jung

College of Engineering and Computing

Andrés G. Gil

Vice President for Research and Economic Development

And Dean of the University Graduate School

Florida International University, 2016 
(C) Copyright 2016 by Nahid Vesali Mahmoud

All rights reserved. 


\section{DEDICATION}

I dedicate this dissertation to my beloved family, who made this work possible. 


\section{ACKNOWLEDGMENTS}

I have been guided and supported by a number of people during my Ph.D. journey, whom I would like to mention here.

To start with, my grateful thanks are to my dissertation co-advisor Dr. M.Emre Bayraktar, who has guided me throughout my research process. He has encouraged and challenged me to accumulate my knowledge in achieving my dissertation goals. Without his precious support and understanding, I would have not been able to complete my Ph.D. studies. I also express my gratitude to him for giving me the opportunity to teach courses, He has been extraordinarily helpful during my difficult times as well as in shaping my career goals.

I would like to specially thank Dr. Xia Jin for serving as a committee member as well as my Co-Major Advisor. Her guidance, efforts and understanding have been valuable throughout my research. I would also like to extend my gratitude to the other members of my dissertation advisory committee, Dr. Young H. Kwak, Dr. Berrin Tansel and Dr. Arindam G. Chowdhury, for their time and guidance during my research.

I would also like to express my sincere appreciation of the Department of Civil and Environmental Engineering, as well as the FIU School Construction, who provided me with the opportunity to pursue my Ph.D. at Florida International University. My special thanks goes to Dr. Irtishad U. Ahmad, Director FIU School of Construction, for his continuous understanding and support. I would also like to thank all the faculty, staff members, and fellow students at the FIU School of Construction.

I would also like to indicate my appreciation of FDOT District Six former Secretary, Mr. Gus Pego, and his staff, and also concessionaire of Port of Miami Tunnel, 
who helped me in collecting data and implementing case study. I would also like to thank Bureau of Transportation Planning, Michigan Department of Transportation, and specially the DCRI project team, who helped me in completing my case study.

Words cannot express how grateful I am to my mother-in law, father-in-law, my mother, and father for all of their sacrifices endless love. Without my parents' unconditional love, supports and encouragements, I wouldn’t be able to complete my $\mathrm{PhD}$ journey or anything that I have achieved. My thanks are also extended to the remaining of my family.

Finally yet importantly, my tremendous and deep thanks goes to my beloved husband, Mostafa Batouli, who motivated, supported and cared about me in all situations. His presence and belief in my work has provided me with great strength. At the end, I must acknowledge with heartfelt gratitude to my lovely daughter, Nila, who brought lots of joy and happiness with her birth and patiently accepted a part-time mum during past seventeen months. 


\begin{abstract}
OF THE DISSERTATION
A COMPREHENSIVE DECISION SUPPORT FRAMEWORK

IN THE FRONT-END PHASE OF MAJOR TRANSPORTATION PROJECTS
\end{abstract}

\author{
by
}

Nahid Vesali Mahmoud

Florida International University, 2016

Miami, Florida

\title{
Professor M. Emre Bayraktar, Co-Major Professor \\ Professor Xia Jin, Co-Major Professor
}

Identifying the best project alternative is a critical challenge facing major transportation projects (MTPs) at the front-end phase. The increasing complexity and dynamism of MTPs have imposed substantial uncertainties and subjectivities in the decision-making process. Despite the efforts made in previous studies, a stochastic framework to facilitate the comprehensive assessment is still missing.

In this research, a stochastic decision support framework has been developed to cope with the considerable uncertainties in MTPs. The features of the proposed decision support framework are achieved by using the Bayesian belief network modeling technique to provide a comprehensive registry of the relevant decision factors, establish the interrelationships between these decision factors, and consequently quantify uncertainties of decision indicators. The calculated probabilities for decision indicators have been interpreted to a satisfaction level of stakeholders based on their constraints as a multi-criteria decision model. A Monte Carlo simulation has been conducted to simulate a real condition using the decision indicators probability as input. Finally, MTP 
alternatives prioritized according to the anticipated satisfactory gained among various stakeholders. The created framework is used in a preliminary alternative assessment for case study related to Detroit River International Crossing project. The case study investigates the decision-making of key stakeholders related to prioritization of alternative projects for a new access between Detroit, US and Winsdor, Canada. The project team verified applicability of the model. The developed framework and the case study highlight the significance of identification of a stochastic project alternative assessment method. The proposed framework provides decision-makers with a decision support tool to facilitate front-end phase of MTPs. 


\section{TABLE OF CONTENTS}

CHAPTER

PAGE

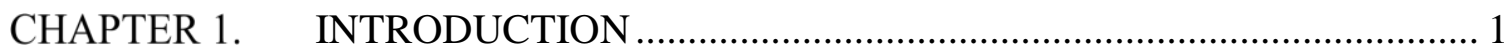

1.1 Background and Motivation......................................................................... 1

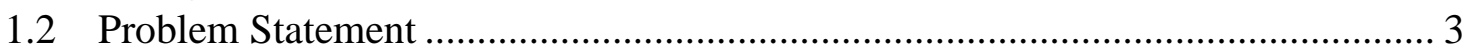

1.3 Challenges in Front-end Phase of Major Transportation Projects ........................ 5

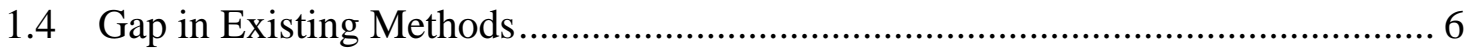

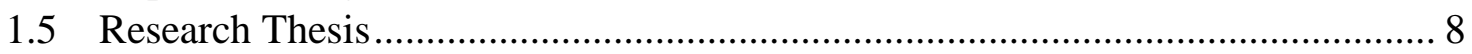

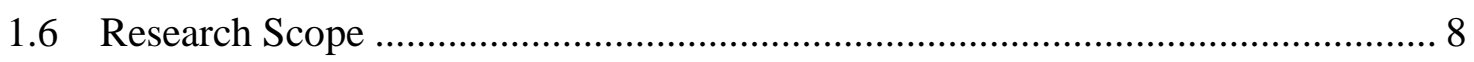

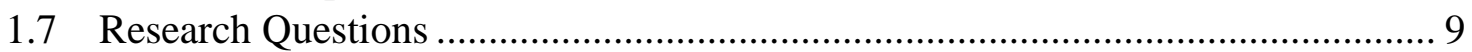

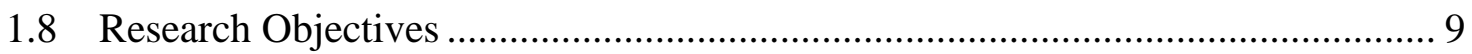

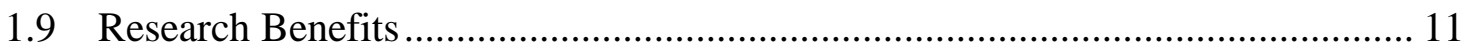

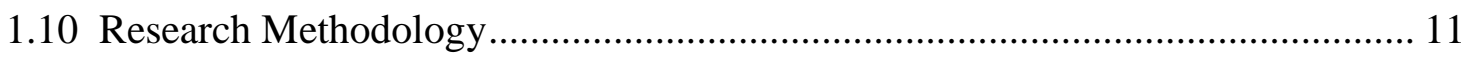

1.10.1 State-of-the-Practice of the Front-end Phase of Major Transportation

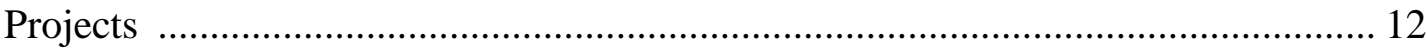

1.10.2 Development of the Decision Support Framework for the Front-end Phase of Major Transportation Projects.............................................................................. 13

1.10.3 Application and Validation of the Decision Support Framework for the Front-end Phase of Major Transportation Projects....................................................... 15

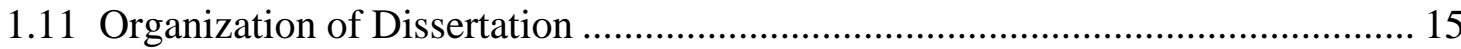

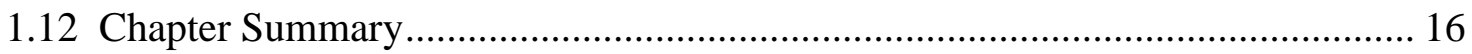

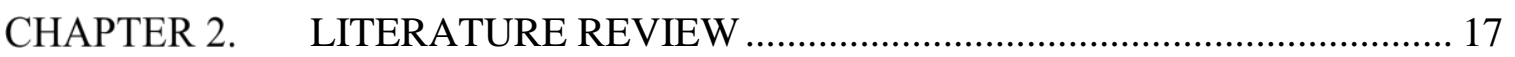

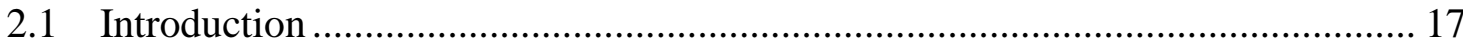

2.2 Importance of Major Transportation Projects (MTPs) .......................................... 17

2.3 Front-End Phase in Major Transportation Projects .............................................. 19

2.4 Decision-Making for Alternative Selection in MTPs .......................................... 24

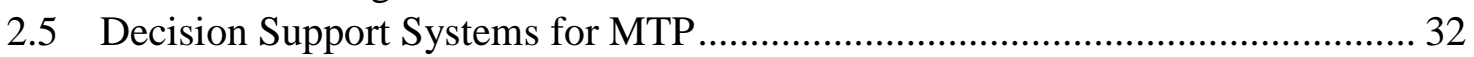

2.6 Gap in Existing Methods of Front-end Phase Decision Making in MTPs........... 36

2.7 Bayesian Belief Network .............................................................................. 37

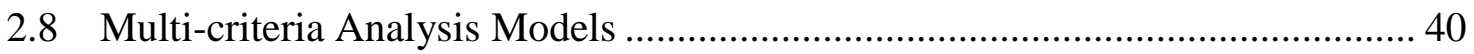

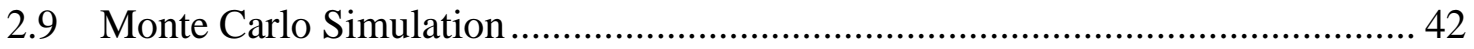

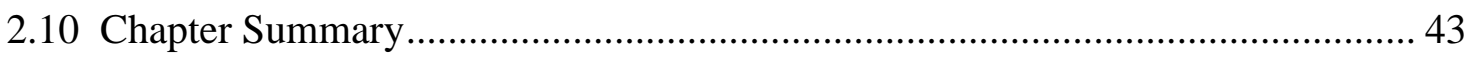

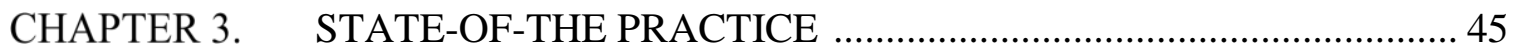

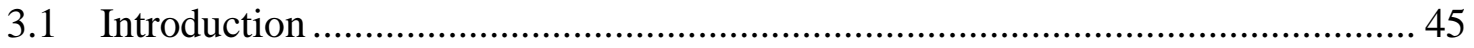

3.2 Legislations and procedures for decision making in major transportation

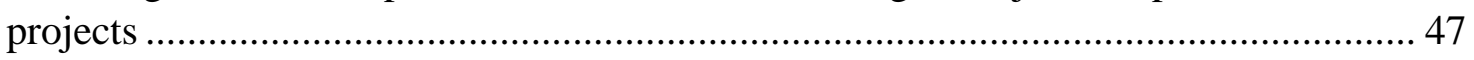

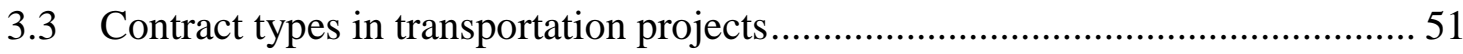


3.4 Importance of public participation in decision making process ............................ 52

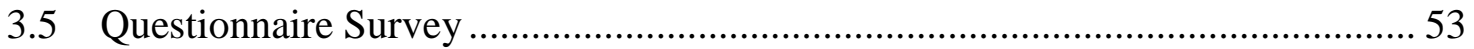

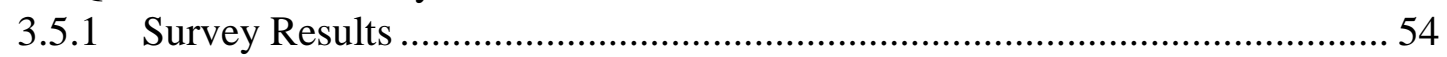

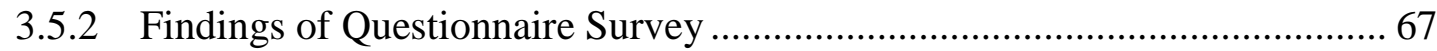

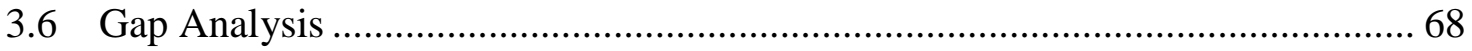

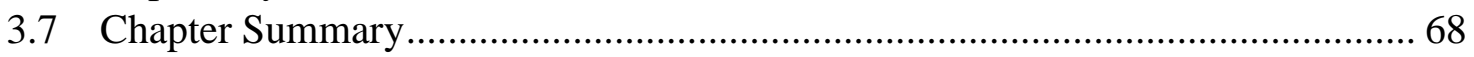

CHAPTER 4. CONCEPTUAL FRAMEWORK DEVELOPMENT ………...............69

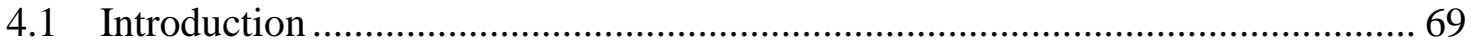

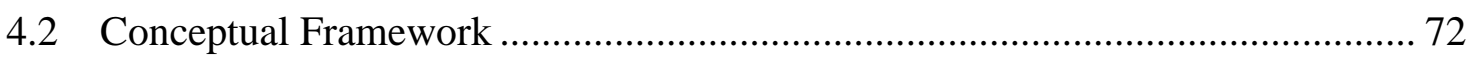

4.2.1 Alternative Identification Module ............................................................ 72

4.2.2 Alternative Assessment Module ................................................................. 74

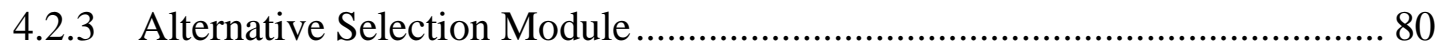

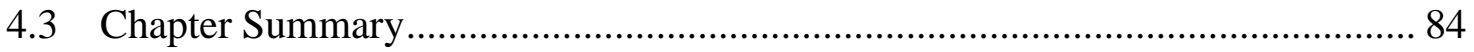

CHAPTER 5. DEVELOPMENT OF FINAL FRAMEWORK FOR

DECISION-MAKING IN THE FRONT-END PHASE OF MTPS ................................ 86

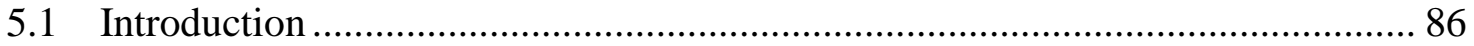

5.2 The Bayesian Belief Network Structure.......................................................... 87

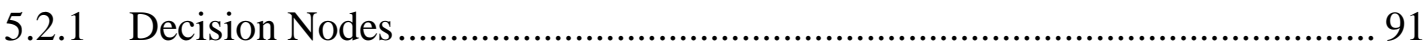

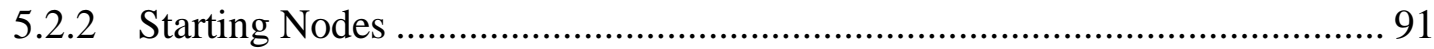

5.2.3 Intermediate Nodes............................................................................... 91

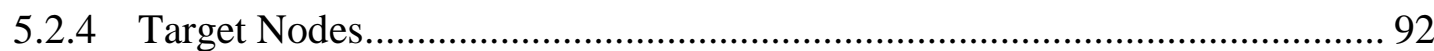

5.3 Monte Carlo Simulation and Multi-criteria Decision-making ............................ 93

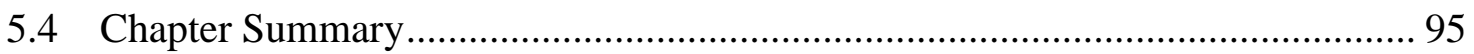

CHAPTER 6. DECISION SUPPORT FRAMEWORK APPLICATION AND

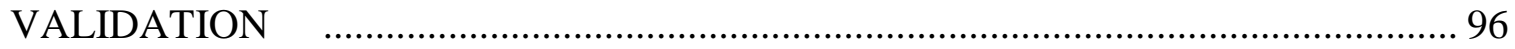

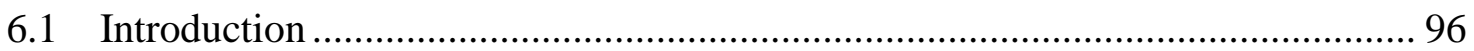

6.2 Case One: Port of Miami Tunnel Project …………............................................ 97

6.3 Case Two: Detroit River International Crossing ................................................. 109

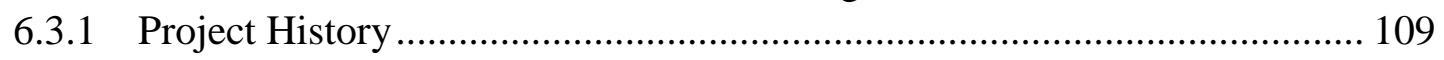

6.3.2 Bayesian Belief Model Customization for DRIC project............................ 112

6.3.3 Application of Alternative Selection Module for DRIC project ................. 126

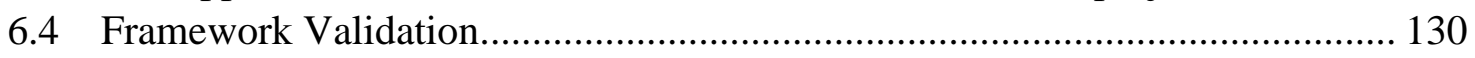

6.4.1 Sensitivity Analysis of the Bayesian Belief Network ................................ 132

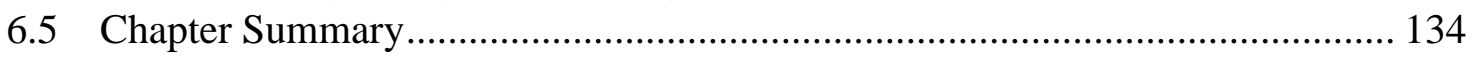

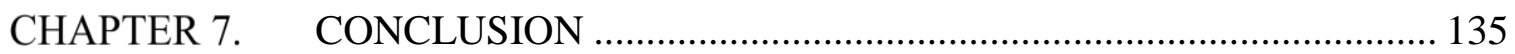

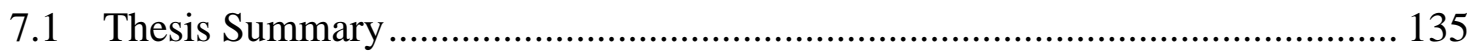




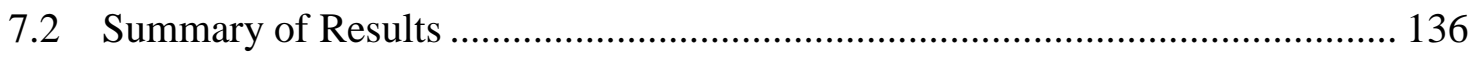

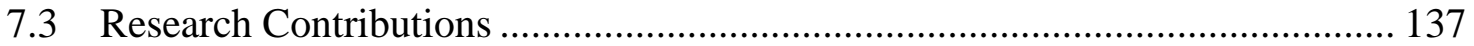

7.4 Limitations and Future Research Potential ................................................. 140

REFERENCES....................................................... 141

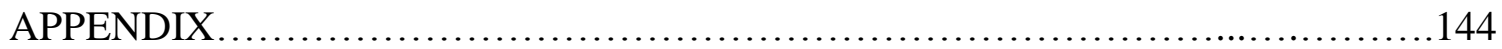

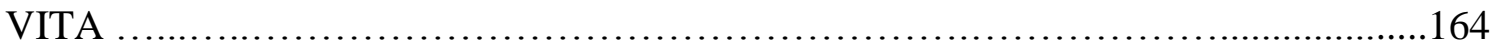




\section{LIST OF TABLES}

TABLE

PAGE

Table 1-1: Front-End Duration of Various Major Transportation Projects ....................... 3

Table 2-1: Transportation project assessment criteria in different methods .................... 30

Table 2-2: Existing Decision Support Systems for MTPs .......................................... 35

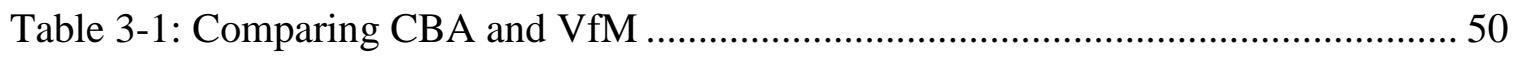

Table 3-2: Importance level of decision-making Decision Indicators ............................. 57

Table 3-3: Importance level of critical factors under "Economic development" ............ 59

Table 3-4: Importance level of critical factors under "Social impacts"......................... 60

Table 3-5: Importance level of critical factors under "Environmental impacts" ............. 61

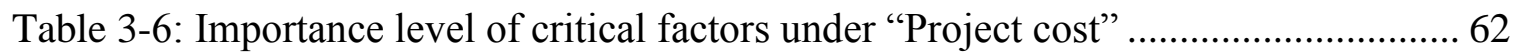

Table 3-7: Importance level of critical factors under "Transportation benefits"............. 63

Table 3-8: Importance level of critical factors under "Technical issues" ........................ 64

Table 3-9: Importance level of critical factors under "Financial issues"........................ 65

Table 3-10: Importance level of critical factors under "Political issues" ....................... 67

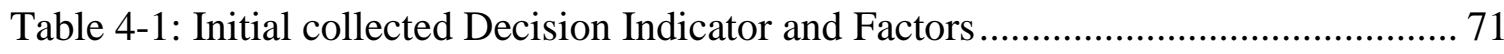

Table 5-1: Dependency and relationship matrix of factors ....................................... 88

Table 5-2: Example stakeholders' satisfaction threshold matrix ................................. 93

Table 6-1: Node probability table for "Improve business productivity" ....................... 101

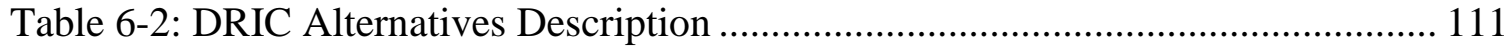

Table 6-3: Modified decision nodes and related node states ..................................... 113

Table 6-4: DRIC project alternatives- BBN scenarios ............................................ 115

Table 6-5: Decision node probability table for DRIC project ................................... 117

Table 6-6: Starting node probability table for DRIC project .................................... 118

Table 6-7: Node state probability of decision indicators for 3 alternatives ................... 121

Table 6-8: Stakeholders' satisfaction threshold matrix for DRIC project ..................... 128 
Table 6-9: Percentage of satisfactory runs of each alternative in respect to

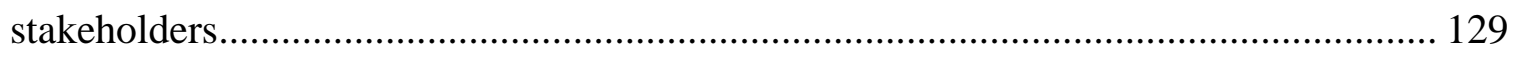

Table 6-10: Alternative desirability index of three alternatives for DRIC project ......... 130

Table 6-11: Rationale and method of assessment used in the evaluation...................... 131

Table 6-12: Summary of evaluation of transportation alternatives .............................. 132

Table 6-13: Most influencing factors of decision indicators for DRIC project.............. 133 


\section{LIST OF FIGURES}

FIGURE

PAGE

Figure 1-1: Typical phases in a project's life cycle (Samset 2008) ................................ 2

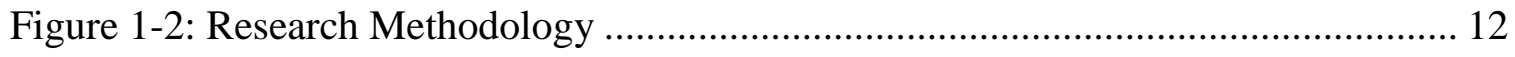

Figure 2-1: Steps of Front-end Phase in Major Transportation Projects ........................ 21

Figure 2-2: Front-end phase duration of various Major Transportation Projects ............ 24

Figure 2-3: Major components of Cost-Benefit Analysis (Lee 2002) ........................... 26

Figure 2-4: Example Bayesian Belief Network .......................................................... 39

Figure 3-1: Assigned importance level for each Decision Indicator .............................. 58

Figure 3-2: Assigned importance level of Critical Factors under "Economic

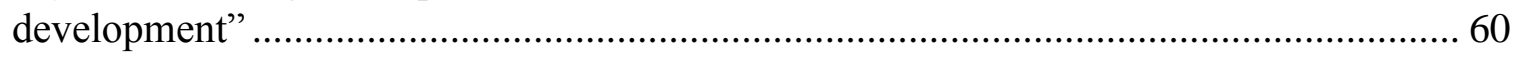

Figure 3-3: Assigned importance level of Critical Factors under "Social impacts"........ 61

Figure 3-4: Assigned importance level of Critical Factors under "Environmental

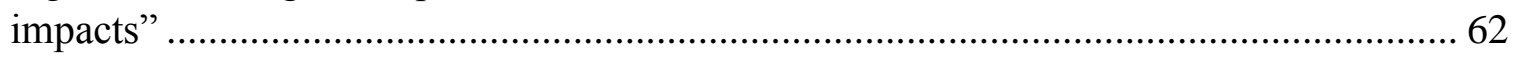

Figure 3-5: Assigned importance level of Critical Factors under "Project cost" ............ 63

Figure 3-6: Assigned importance level of Critical Factors under "Transportation

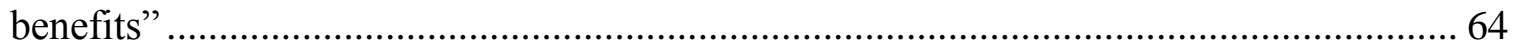

Figure 3-7: Assigned importance level of Critical Factors under "Technical issues" ...... 65

Figure 3-8: Assigned importance level of Critical Factors under "Financial issues" ....... 66

Figure 3-9: Assigned importance level of Critical Factors under "Political issues" ........ 67

Figure 4-1: The proposed framework modules ......................................................... 73

Figure 4-2: Steps within Alternative Assessment Module ............................................ 75

Figure 4-3: Schematic Bayesian belief network for decision indicators ....................... 76

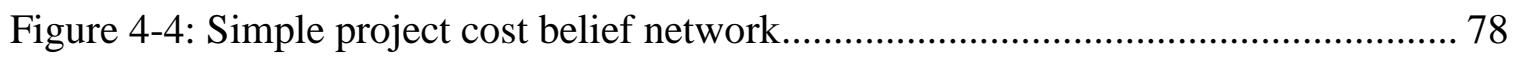

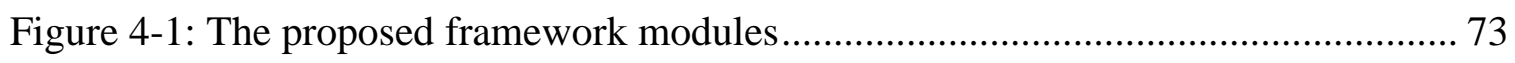

Figure 4-5: An example of stakeholders' satisfaction threshold mtrix .......................... 83

Figure 4-6: Steps within Alternative Assessment Module ......................................... 84

Figure 5-1: Generic cause and effect BBN with different levels of nodes ..................... 90 
Figure 6-1: Generic relationships of economic development factors ........................... 98

Figure 6-2: An underwater Tunnel connected to I-395 from Watson Island.................. 100

Figure 6-3: A Bridge with shoreline connection to I-395 ......................................... 100

Figure 6-4: Calculated conditional probabilities for BBN nodes of Economic

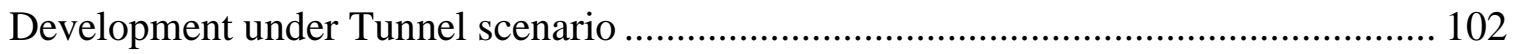

Figure 6-5: Calculated conditional probabilities for BBN nodes of Economic

Development under Bridge scenario................................................................. 103

Figure 6-6: Generic BBN for "Environmental Impacts” Decision Indicator ................ 104

Figure 6-7: Calculated Probability for Tunnel Alternative ......................................... 105

Figure 6-7: Calculated Probability for Bridge Alternative ........................................ 106

Figure 6-9: A sample node probability table ............................................................ 107

Figure 6-10: Node probability table for the "Quality of trip" node of DRIC project ..... 119

Figure 6-11: Economic development state probability for DRIC project...................... 122

Figure 6-12: Social development state probability for DRIC project........................... 123

Figure 6-13: Protect natural environment state probability for DRIC project............... 123

Figure 6-14: Transportation benefits state probability for DRIC project ...................... 124

Figure 6-15: Technical feasibility state probability for DRIC project.......................... 124

Figure 6-16: Project cost state probability for DRIC project.................................... 125

Figure 6-17: Financial feasibility state probability for DRIC project.......................... 125 


\section{CHAPTER 1. INTRODUCTION}

\subsection{Background and Motivation}

The need for new and updated infrastructure has grown greatly all around the world in the last decades. Rough estimates from the Organization for Economic Cooperation and Development (OECD) Infrastructure Project suggest that annual investment requirements for road and rail together are around an average of $0.75 \%$ of world GDP. Considering that the estimation does not cover other transportation infrastructures such as ports and airports, the ratio would rise further if it includes all types of transportation infrastructure (OECD 2007). This investment is required due to several reasons including: population increase, migration flows toward cities, deterioration of existing aging assets, and the globalization of supply chains (Gil and Beckman 2009; Bruzelius et al. 2002).

Major Transportation Projects (MTPs) are a particular class of high profile infrastructure assets which typically draw more attention. These projects attract a high level of public attention and political interest not only due to their considerable cost, but principally because of their substantial and long lasting direct and indirect development impacts on communities, environments economies and institutions at local, regional, national and international levels. Especially in the last two decades many countries, including developing and industrialized countries, take major transportation projects as an important tool to raise the status in globalization (Jia et al. 2011). Most of the famous

transportation infrastructure projects around the world are qualified as capital transportation infrastructure project, such as English Channel between UK and France; 
Central Artery/Tunnel in Boston, MA; Miami Port Tunnel, FL; High-Speed Rail between San Francisco and San Diego, CA; Alaskan Way Viaduct Replacement Project in Seattle, WA; Marmaray Tunnel in Istanbul, Turkey.

In projects with poor performance, research indicates that the problem is strongly associated with the decisions in the earliest phase, i.e., the front-end phase, where the initial idea was conceived (Figure 1-1). What happens during the front-end phase is essential for the project's success. Decisions during the front-end phase will clearly have implications for planning and implementation of the project. A study by the World Bank in 1997 , based on a review of as many as 1125 projects, concluded that $80 \%$ of the projects with a satisfactory 'quality at entry' were successful while only $35 \%$ of those with unsatisfactory quality were successful (Samset 2008).

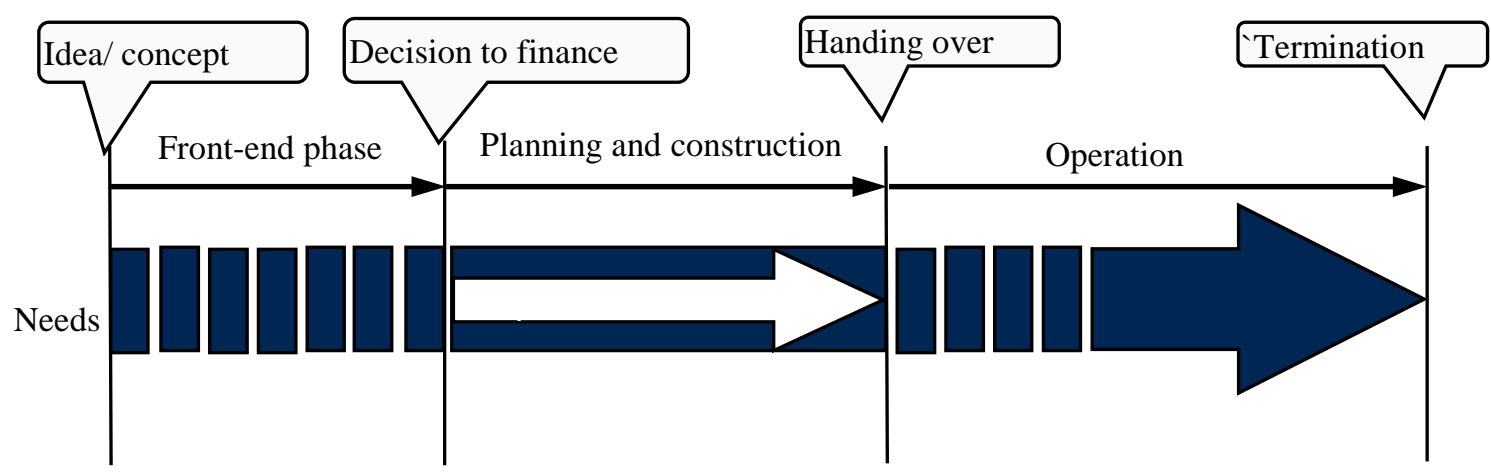

Figure 1-1 Typical phases in a project's life cycle (Samset 2008)

In major transportation projects, the front-end phase presents a complex and timeconsuming decision-making process aimed to generate, consolidate, and analyze relevant information and to arrive at the final solution. As shown in Table 1-1, it is not uncommon for major transportation projects that the front-end phase takes years even decades. 
In light of the introduction, the ultimate objective of the research is to develop a holistic decision support framework addressing the front-end phase of major transportation projects to ensure that the assessment of project alternatives is conducted in a systematic, transparent and explicit way.

\section{Table 1-1: Front-End Duration of Various Major Transportation Projects}

\begin{tabular}{|c|c|c|}
\hline $\begin{array}{l}\text { Port of Miami } \\
\text { Tunnel }\end{array}$ & $\begin{array}{l}\text { Vehicular access between port of Miami } \\
\text { and Miami is needed. }\end{array}$ & $1981-1990$ \\
\hline $\begin{array}{l}\text { New East-West } \\
\text { Railway under } \\
\text { London }\end{array}$ & $\begin{array}{l}\text { To cope with London's forecast } \\
\text { population and economic growth, and } \\
\text { allow existing suburban east-west rail } \\
\text { services to run through central London }\end{array}$ & $\begin{array}{l}\text { first public discussions date: } 1974 \\
\text { and plan was finally approved and } \\
\text { received funding: } 2008\end{array}$ \\
\hline $\begin{array}{l}\text { Terminal } 5 \text { (T5) } \\
\text { at Heathrow } \\
\text { Airport }\end{array}$ & $\begin{array}{l}\text { Increase in travel demand in London in } \\
1980 \text { s }\end{array}$ & $\begin{array}{l}20 \text { years between initiating the } \\
\text { planning application and the } \\
\text { opening of the first phase of the } \\
\text { new terminal in } 2008\end{array}$ \\
\hline $\begin{array}{l}\text { Central } \\
\text { Artery/Tunnel } \\
\text { project in Boston }\end{array}$ & $\begin{array}{l}\text { High congestion due to unexpected } \\
\text { number of users and excessive number of } \\
\text { exits }\end{array}$ & $\begin{array}{l}\text { In the } 1980 \text { 's, initial plan was } \\
\text { suggested. The final } \\
\text { Environmental Impact Report was } \\
\text { submitted and approved in } 1990 .\end{array}$ \\
\hline $\begin{array}{l}\text { High-Speed Rail } \\
\text { in California }\end{array}$ & $\begin{array}{l}\text { Increases in travel demand in CA, need } \\
\text { for link the major cities with predictable } \\
\text { and consistent travel times. }\end{array}$ & $\begin{array}{l}\text { Proposed in 1980s, First section } \\
\text { construction began in } 2010\end{array}$ \\
\hline $\begin{array}{l}\text { Alaskan Way } \\
\text { Viaduct } \\
\text { Replacement } \\
\text { Project (AWV) }\end{array}$ & $\begin{array}{l}\text { Feb 28, } 2001 \text { Nisqually earthquake } \\
\text { severely damaged 2-level State Route } 99 \\
\text { highway in Seattle. It should be replaced. }\end{array}$ & $\begin{array}{l}\text { First call for replacement options } \\
\text { in July } 2001 \text { and in early } 2008, \\
\text { final alternatives were identified. }\end{array}$ \\
\hline
\end{tabular}

\subsection{Problem Statement}

Most major transportation projects face higher cost than the estimated amount and longer time than the initial schedule. Many construction industry experts indicated that efforts during the early stages of a project significantly affects project success (Gibson Jr et al. 1995). This implies that the aforementioned problems initially have been formed in the project planning stage. Planning of major transportation infrastructure projects are challenging, especially in early stages, due to their complexity, inherent uncertainty and 
encompassing several stakeholders. The initial choice of project concept, which is one of the imperative decisions with significant impact on a project's future performance, occurs in this stage (Williams and Samset 2010; Priemus 2010a). The early stage of project planning development, which is called the Front-end phase in this study, is a crucial stage for major transportation projects. Each deficiency in this phase results in negative impacts in final project success. Common observed deficiencies of front-end phase are: 1) A transparent, explicit and systematic procedure is rare in this phase and leads to taking long time; 2) Project level uncertainty often is not considered in the front-end phase and alternative appraisal; 3) Since several stakeholders with conflicted interests are incorporated in major transportation projects decision making process, alternative selecting is a complicated process.

Front-end phase, also referred to as pre-project planning, is an important subset of planning stage, which is "the process of developing sufficient strategic information for owners to address risk and decide whether to commit resources to maximize the chance for a successful project" based on pre-project planning research team of the Construction Industry Institute (ClI) definition. Front-end planning phase is also called feasibility analysis, conceptual planning, and programming (Gibson Jr et al. 1995). Front-end phase includes all the tasks from the time that the initial idea is conceived, until taking final decision to finance and beginning of detailed design (Williams and Samset 2010). It begins with a need appraisal for project and ends with deciding about optimum solution for the proposed project (Gibson Jr et al. 2006). 


\subsection{Challenges in Front-end Phase of Major Transportation Projects}

Reviewing front-end phase in several major transportation projects implies that a transparent, functional and systematic procedure that sets out what needs to be firmly established (program, minimum performance, public value) is scarce in construction industry (Priemus 2010a). This shortage leads in consuming large amount of time and cost in front-end phase. It is not unusual for major transportation projects to take ten years in the front-end phase. Many projects have been taken far longer (even twenty years) if significant community or environmental issues were involved. One of the examples is Port of Miami Tunnel; it took about 9 years from first need arising for vehicular access to Port of Miami in 1981 till its preliminary design in 1990. Taking long time in initial steps often results in significant changes in project scope and also need for the whole project. Especially in the case of infrastructure projects, which usually intend to satisfy urgent regional needs, this kind of delays may cause problems. Delayed projects exacerbate the social and economic costs of congestion and safety problems. Technological advancements happening during the front-end phase can create new needs or eliminate previous needs.

Another important problem related to the front-end phase of major transportation projects is lack of incorporating uncertainties in the decision making process. High level of complexity in major transportation projects increases the uncertainty in this kind of projects. Reviewing the literature and documents of constructed major transportation projects has revealed either altering the selected project alternative during the front-end phase or consequent changes in the construction phase. The current methodologies for 
alternative selection in major transportation projects often applied a deterministic approach based on "best guess" estimation of the input variables in the analysis.

The other main problem in dealing with front-end phase of major transportation projects is facing large number of stakeholders with contrasting interests. Finding an alternative to meet goals of all stakeholders is a difficult task that needs considering behavioral impact of each party in decision-making process. Failure to consider the dynamic relationships between the stakeholders in the front-end phase of major transportation projects leads to unexpected problems in the future phases.

The three basic problems described above indicate the need for improving the front-end decision making process in major transportation projects. This mentioned problems will be addressed by the comprehensive decision support framework to be developed over this research.

\subsection{Gap in Existing Methods}

Whether or not to construct a major transportation infrastructure is a major decision that should be deliberated carefully. The most common methodology applied to the evaluation of transportation project alternatives in the US as well as Europe has been conventional Cost-Benefit Analysis (CBA), which, supported by traffic and impact model calculations, provides the decision makers with a monetary assessment of the project's feasibility. This is a deterministic approach based on "best guess" estimation of the input variables in the analysis. Although widely used, CBA is also contested in literature as being inadequate for appraising transport-related plans, especially because of its too much focus on how infrastructure can help solve traffic bottlenecks (i.e. decreased travel time) and too little on how it can support a vision for spatial economic developments 
(Beukers et al. 2012). Furthermore, the impact of various stakeholders with conflicting interests and the interrelationship among them is not considered in current methods.

Moreover, the public has little understanding of the issues involved and it has become increasingly obvious that the traditional patterns of decision making often do not result in policies and projects that promote a sustainable intermodal system. In addition to the modal focus referred to above, transportation planners and decision makers are not primarily concerned with such considerations as maintaining flexibility or ensuring that genuine public participation takes place. The decision-maker's thinking continues to be dominated by the rational actor model which views planning as a systematic, step-by-step process, which involves such stages as problem definition, value clarification, goal selection, formulation of alternatives, evaluation of alternatives, and selection and implementation of a course of action. Planners and decision makers must marshal all the relevant information. All possible alternative solutions are generated and analyzed. The optimal alternative is eventually selected for implementation A rational approach in planning should result in maximizing benefits and minimizing its costs, but this has frequently not been the case.

To identify gaps that exist between theory and practice, the approach embodied in the proposed transportation decision-support framework is compared with current metropolitan transportation planning and decision-making processes in the U.S. The framework is then used to consider how the U.S. federal government might move the nation's transportation system towards sustainability 


\subsection{Research Thesis}

The front-end phase of major transportation projects is a complicated and timeconsuming process. Several stakeholders engage in project scope development that happens in this phase. Lack of a transparent and systematic procedure for this phase exacerbates its complexity. The ideas to meet project needs evolve out of work done in this phase. Making a decision to select the best alternative to implement in the next phases of the project also happens during the front-end phase. Consideration of multiple decision parameters in an alternative selection can be beneficial for owner organizations and governmental agencies. The aforementioned considerations present challenges to the decision makers in major transportation projects. Therefore, it is important to develop a decision support framework that clarifies the process of the front-end phase and allows evaluating multiple decision parameters with respect to the organization priorities. Simulation of the decision-making process by abstracting the stakeholders' behavior is a further step to facilitate the decision-makers in the front-end phase.

\subsection{Research Scope}

The scope of this research is any major transportation infrastructure such as surface routes (highways, roads, and railways), airports, tunnels, bridges, ports, etc. which often cost more than $\$ 1$ billion and consume large amount of resources. Primarily focus of this research is in front-end phase, which begins with need identification and ends with selecting the best alternative for implementation to solve the existing problem. 


\subsection{Research Questions}

Referred to the problems described above and for the purposes of research proposed in this project, the following research questions will be addressed:

1. What are the appropriate Decision Indicators for evaluation and prioritization of project alternatives in major transportation projects?

2. What are the critical tangible and intangible factors that could influence the decision indicators?

3. What are the interrelationships between the identified critical factors and decision indicators?

4. What criteria and techniques should be used to quantify the subjective assessment of the effect of critical factors on the decision indicators?

5. Which stakeholders are involved in the major transportation projects and how their influence on decision-making process can be modeled?

6. Given the quantity of decision indicators and behavior pattern of stakeholders, what methodology and practices can lead to the final ranking of project alternatives in major transportation projects?

\subsection{Research Objectives}

This research develops a coherent, well-structured, flexible, straight forward decision support framework, addressing the front-end phase of major transportation projects to ensure that evaluation and prioritization of project alternatives is conducted in a systematic, transparent and explicit way taking into account all the relevant decision factors and criteria. Based on the background and problem statement provided in the 
preceding sections, the framework is designed in such a way that facilitates streamlining of the process to save time as well as incorporating uncertainty to the assessment of the monetary and non-monetary decision factors playing a role in the decision making process of major transportation projects. Also, as mentioned earlier, the created framework reflected the impact of multiple stakeholders in the decision-making process with various behaviors and attempted to simulate the real decision-making environment. Moreover, the framework is developed in such a way that can be easily implemented into software and integrated with the existing systems while facilitating effective decisionmaking process.

The main objectives of this study are to:

1. Investigate the issues related to the front-end phase in major transportation projects.

2. Explore the assessment models and available techniques of alternative evaluation in the front-end phase of major transportation projects.

3. Determine the appropriate decision indicators for evaluation and prioritization of project alternatives in major transportation projects.

4. Identify the critical tangible and intangible factors that could influence the decision indicators.

5. Establish the general influence pattern of the identified critical factors on decision indicators.

6. Abstract the constraints and rules of various stakeholders/agents engaged in the frontend phase of major transportation projects.

7. Simulate the real decision making process by modeling the satisfaction of various stakeholders. 
8. Create the holistic stochastic decision support framework for the entire process of the front-end phase in major transportation projects

9. Apply the framework to a real world case.

\subsection{Research Benefits}

The final outcome this research is a prototype decision support framework that can facilitate the decision making process in the front-end phase of major transportation projects. This framework can explicitly guide the decision makers with providing important factors and decision indicators and their interrelationship. Furthermore, it addresses the influence of multiple stakeholders and attempts to quantify their subjective impacts.

\subsection{Research Methodology}

After establishing the objectives and expected outcomes, this research has been done through three phases: 1) State-of-the-Practice of the Front-end Phase of Major Transportation Projects; 2) Development of the Decision Support Framework for the Front-end Phase of Major Transportation Projects; and 3) Application and Validation of the Created Framework. Figure 1-2 depicts the phases and tasks flow in this research. The tasks of these phases are described below. 


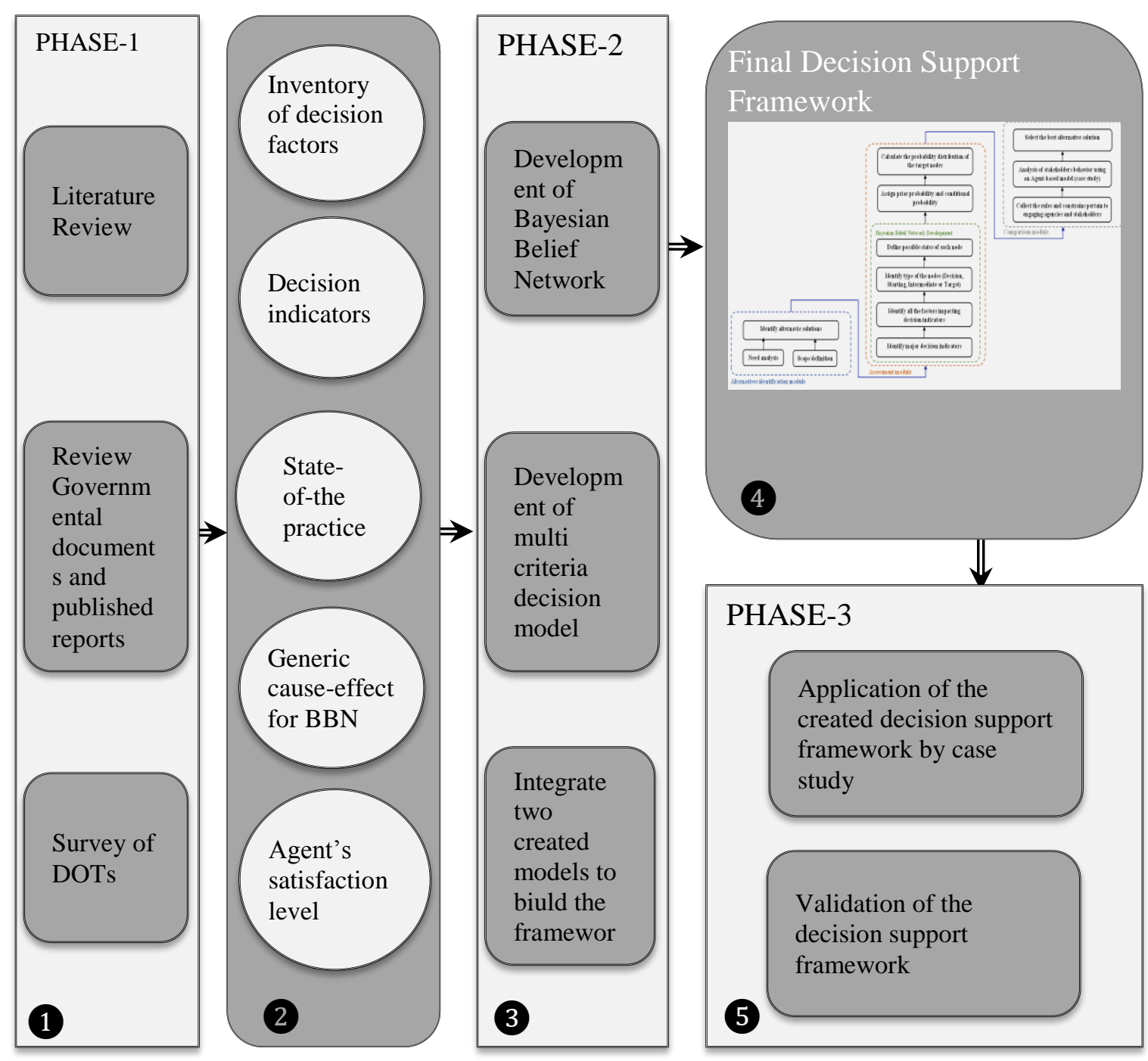

Figure 1-2: Research Methodology

\subsubsection{State-of-the-Practice of the Front-end Phase of Major Transportation Projects}

The tasks through Phase-1 are conducted to establish the state-of-the-practice of major transportation projects in the front-end phase and to identify the major concepts, definitions, principles, processes, and tools relevant to this phase. The primary objective of Phase-1 is to clarify the procedures of the front-end phase of MTPs. Accomplishment of the Phase-1 objective are require the first three tasks of the research, which are described in detail as follows: 
Task-1: Literature Review

The first task after identification of the research objectives and scope was an extensive literature review of academic publication including journal articles and conference papers. This task had been done to establish the knowledge base and concept of major transportation projects, and existing decision support frameworks and alternative selection tools and methods. The important factors influencing the decision making process of major transportation projects were also identified through this task.

Review of governmental agencies' published documents and reports

The literature review followed by a comprehensive review through a variety of credible sources, including the reports and technical documents published by FHWA, State Departments of Transportation, Metropolitan Planning Organizations (MPO), U.S. Environmental Protection Agency, and other similar agencies in Europe.

Task-2: Survey of the State DOTs and MPOs

To obtain the most recent information about the existing activities and processes in the front-end phase of MTPs, a questionnaire survey had been conveyed. The questionnaire was designed based on the findings of the first two tasks. The target respondents of the survey were the relevant state DOTs and MPOs staffs all over the US.

\subsubsection{Development of the Decision Support Framework for the Front-end Phase of Major Transportation Projects}

The objective of the second phase was to develop a holistic decision support framework for front-end phase of MTPs. The framework should be flexible such that it can be effectively scaled and applied for differing needs of transportation agencies and other decision-making organizations. All the important categories in decision-making and 
alternative selection methods were combined together to form the framework. This phase had been conducted through two tasks including: 4) Development of Bayesian Belief Network for the front-end phase of MTPs; and 5) Development of Monte Carlo Simulation and Multi criteria Decision-making Model for based on stakeholders' preferences in the front-end phase.

Task 3: Development of Bayesian Belief Network for the front-end phase of MTPs

The outcomes of the previous phase of the research have been used as the building blocks of the BBN. An inventory of influencing factors and a list of decision indicators regarding front-end phase of MTPs were compiled over the course of state-ofthe practice phase. Then the generic cause-effect network had been formed based on refined list of the factors and decision indicators, which resulted in the general schematic BBN. The probability table for each factor was also filled as a part of this task (these probability values are unique for each specific MTP case). The outcome of this task was a list of decision indicators with their calculated probability distribution.

Task 4: Development of Monte Carlo Simulation and Multi-criteria Decision-making Model in the front-end phase

To address the complexity of decision making in the front-end phase due to multiple stakeholders along with uncertain upcoming conditions, a Monte Carlo simulation model has been developed in this step. The behavior of different engaged parties were documented and abstracted as constraints, and random result of the BBN is converted to the satisfaction level stakeholders. Then, using a rule to accept or reject of an alternative, defined by the decision-makers, the optimum alternative can be obtained at the end of this task. 


\subsubsection{Application and Validation of the Decision Support Framework for the Front-end Phase of Major Transportation Projects}

In the last phase of the research the concept and methodology was demonstrated by applying the framework to an actual project (case study). Based on the data from this case, the framework was modified and customized and the obtained results was analyzed and compared with the real world data. After application, based on data availability and feedback from various sources, the framework was validated.

\subsection{Organization of Dissertation}

This dissertation outlines in 7 Chapters. Chapter 1 provides an introduction to the study and includes the background, problem, research questions and objectives, as well as scope and benefits of the study. The research methodology and structure of the dissertation are also outlined in this chapter. Chapter 2 reviews published papers and any other academic sources related to the front-end phase process, specifically for major transportation projects. A brief introduction of modeling methods and tools is also provided in this chapter. Chapter 3 presents the current state-of-the practice founded from published governmental reports and documents as well as information gathered through an online questionnaire survey. Chapter 4 formulates the conceptual framework the final decision support framework based on literature review and survey results. Chapter 5 provides extensive step-by-step details regarding Bayesian Belief Network development and Multi-criteria Decision Model development respectively. The application of final decision support framework has been provided in chapter 6 along using the data from an actual project (Detroit River International Crossing). The validation of the framework is also provided in this chapter. Finally, Chapter 7 concludes the research summary, 
contribution and limitation. The recommendations for future researches are also provided in this chapter.

\subsection{Chapter Summary}

An effective decision making approach in front-end phase of MTPs that targets selecting the best alternative solution is challenging because of the fundamental integration of inherent uncertainty and role of multiple stakeholders with conflict interests. Conventional methods such as cost-benefit analysis yield only part of the problem. To address the complexities of such coupled systems, a hybrid agent-based modeling approach is created to comprehensively simulate the effects of multiple stakeholders with different behavior along with a Bayesian Belief Network to overcome the uncertainty. 


\section{CHAPTER 2. LITERATURE REVIEW}

\subsection{Introduction}

The major objective of this research was to develop a decision support model to facilitate the decision making process of Major Transportation Projects in the front-end phase. As such, the research involved a Major Transportation Project aspect, a front-end phase aspect, a project alternative assessment aspect, and a decision support systems aspect. This chapter presents the findings of a comprehensive literature survey on the abovementioned aspects. The findings regarding the current alternative assessment process will be completed in Chapter 3, along with the discussion of the questionnaire survey. This chapter also offers a brief introduction to Bayesian belief networks as a tool for modeling. Finally, the results of the literature review are presented in the summary part of the chapter.

\subsection{Importance of Major Transportation Projects (MTPs)}

Major infrastructure projects, also referred to as mega or capital infrastructure projects, usually require substantial investment ( $\$ 1$ billion or more) (Li and Guo 2011; Bruzelius et al. 2002) and large amounts of resources that include lots of man hours, materials, and several interlinked stakeholders. Such projects often have long construction durations ( $\mathrm{Li}$ and Guo 2011), as well as long operation times of over 50 years, and generate multiple social impacts. There is considerable uncertainty regarding the major projects due to demand forecasts and cost estimations ( $\mathrm{Li}$ and Guo 2011; Bruzelius et al. 2002). Governments are highly ambitious for these kind of projects since they have magnificent impact on society and will remain in the history (Priemus 2010b), 
therefore, most of the major projects are invested or commissioned by governments (Sun and Zhang 2011). These large-scale projects can be either in national level or international level. Most of the famous transportation infrastructure projects around the world are qualified as capital transportation infrastructure project, such as English Channel between UK and France; Central Artery/Tunnel in Boston, MA; Miami Port Tunnel, FL; High-Speed Rail between San Francisco and San Diego, CA; Alaskan Way Viaduct Replacement Project in Seattle, WA; Marmaray Tunnel in Istanbul, Turkey.

In the U.S., based on the Safe, Accountable, Flexible, Efficient Transportation Equity Act: A Legacy for Users (SAFETEA-LU), signed into law on August 10, 2005, a Major Project is defined as "a project with a total estimated cost of $\$ 500$ million or more that is receiving financial assistance." However, this is not the only specification for MTPs. The FHWA may choose to categorize a project as MTP in situations where the project requires a substantial portion of the State Transportation Agency (STA)'s program resources; has a high level of public or congressional interest; is unusually complex; has extraordinary implications for the national transportation system; even if the total cost is not exceeded $\$ 500$ million. These projects have considerable risks and uncertainty in terms of cost, design, and construction (FHWA Office of Innovative Program Delivery, Project Delivery 2016).

The main distinction of MTPs from other transportation projects is their long period of planning and decision-making and causing contention. The complexity is the main issue in their planning and decision making process (Giezen et al. 2014). The decision-making and planning of a project are usually occurred in the early stage of project life, which is called Front-end phase in this research. 
The next section provides an in-depth description about front-end phase of major transportation projects.

\subsection{Front-End Phase in Major Transportation Projects}

Front-end Phase is the earliest phase of project life and defined as "the process of developing sufficient strategic information with which owners and contractors can address risk and decide to commit resources to maximize the chance for a successful project" (CII 2012). There are also other terms which have been used by industry for front-end planning such as front-end loading, pre-project planning, programming, schematic design, design development or sanctioning. Based on CII definition, this process initiates with conceiving the initial idea and identifying the concept of project to achieve the project objectives, then the feasibility verification which is one of the subphases should have been done. Next step is identifying the alternative ways and final decision that determines whether or not to finance the project. At the end the detailed scope of work should be developed before starting the next phase (CII 2012). Williams and Samset mentioned the front-end phase is when the project exists only conceptually, before it is planned and implemented (Williams and Samset 2010). Typically, the frontend phase process consists of gathering the project team, selecting technology, selecting the project site, developing project scope, and developing project alternatives (Gibson Jr et al. 1995).

Many researches have shown the significant impact of front-end phase on project success. The precisely developed project scope in front-end phase will result in successful project delivery (Gibson Jr et al. 2006; Yun et al. 2012). The implication of the

decisions during this phase for planning and implementation phases of project became 
clear in studies. World Bank reviewed 1125 projects in 1997 and investigated that $80 \%$ of the projects, which were well-defined and well evaluated at the beginning stage, were successful while only $35 \%$ of those with unsatisfactory quality were successful (Samset 2008).

The process of front-end phase can be briefly described as follows. The first step in front-end phase is identifying the need. The demand for a transportation project is influenced by economic, demographic, behavioral, and technological factors as well as by the existence of a specific infrastructure service. Moreover, demand can be changed by the capacity of the transportation where affects journey times and comfort (Hall et al. 2014). The process proceeds by developing the scope of the project, which has been recognized as a critical factor in ultimate success of the project (Gibson Jr et al. 2006). The scope is defined as the final output of project to be delivered on-scheduled and through the functional specification, which can meet the required demand. This step is rather difficult due to many unknown factors and high uncertainty. The objectives of the project are usually set as the benefits for its stakeholders, society and environment all together (Priemus 2010b). Then different ideas to meet the demand through defined objectives are generated and assessed to reach the final plan for the project. The process of front-end phase is depicted in Figure 2-1.

In the United States, the transportation planning process should be considered in the front-end phase. The transportation planning process includes metropolitan and statelevel planning, each of which is required to have short- and long-term transportation improvement programs (TIP). Federal, state, and regional agencies, as well as local governments and citizen groups have done need identification in this phase. Special- 
interest groups also become involved through town hall meetings, public hearings, and other forums. These activities are included in network-level planning which yields a set of selected projects to meet the transportation needs. The suggested project should be consistent with network-level systems planning which includes various aspects such as environmental inventories as well as inputs from the management systems for pavements, bridges, public transportation, intermodal facilities, safety, and congestion (Sinha and Labi 2011).

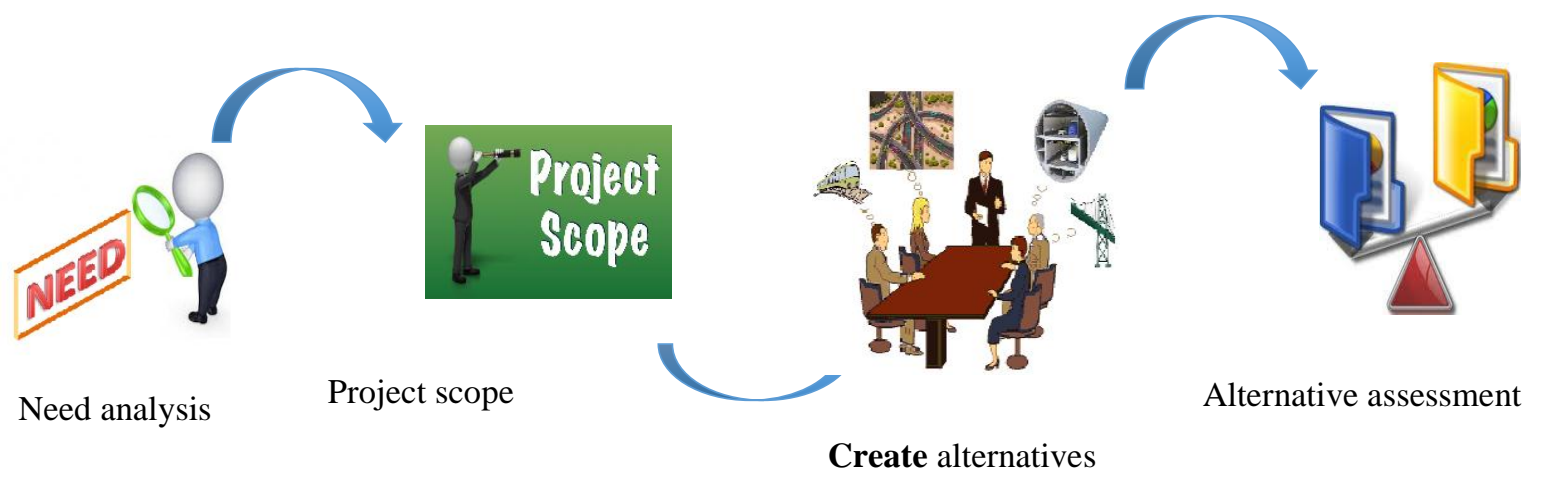

Figure 2-1: Steps of Front-end Phase in Major Transportation Projects

Federal Highway Administration (FHWA) delineates three aspects of project scope, which are required to be defined at the starting point of transportation projects (especially highway projects). The first aspect is geographical scope that includes: Intersections with other forms of infrastructure (road, rail, pipelines, etc.); Entrances and exits; Construction synergies. The second one is functional scope, which determines the tasks of projects and the role of different parties in each task. The last aspect is temporal scope that includes issues affecting the duration of the project such as expected environmental changes (FHWA Office of Innovative Program Delivery, Project Delivery 2016). 
One of the stages of Project Development Process (PDP) for transportation projects in the U.S. is defined as "Project Identification and Scoping" which is very close to the front-end phase defined here. This stage involves a small component of a networklevel plan such as a corridor, link, or node. It includes location planning and commonly should take three to five years, depending on the project complexity. Sinha and Labi (2011) determined following general steps for this stage (Sinha and Labi 2011):

1. Evaluation of existing modal facilities and further study of the need and purpose of the proposed improvement

2. Collection and analysis of social, economic, and environmental data

3. Definition of alternative project corridors, links, or nodes

4. Informal public meetings

5. Draft environmental impact report

6. Location public hearings

7. Final report and environmental impact statement approval

8. Location approval

Some federal laws and regulations should be considered at this stage, which concern ecology, natural resource (i.e., land, water, energy, etc.) conservation, air pollution, historic facility preservation, archeological resources, civil rights, property relocation and acquisition, and other factors. The impact of special-interest groups such as the Sierra Club, the Environmental Defense Fund, and the Center for Law in the Public Interest could be effective at this stage (Sinha and Labi 2011).

But CII established a little different activity sets for front end phase of projects which is more comprehensive and includes following (CII 2012): 
1. Option analysis

2. Scope definition and boundaries

3. Life-cycle cost analysis

4. Cost and schedule estimate

5. Site investigation environmental analysis

6. Process design basis

7. Initial equipment design

8. Space planning, including room data sheets and stacking diagrams

9. Site layout

10. Project execution approach, including project control plan

11. Procurement plan

12. Architectural renderings

13. Approaching submittal package

As mentioned above, many critical decisions of project life have been taken in front-end phase. Therefore, exercise to improve the quality of activities and outcomes of this phase is enticing for researchers and decision makers. An overall review of front-end phase in major transportation projects revealed that it is a lengthy process and it took far more than what is expected (three to five years). There are some examples of MTS which took over 20 years to reach the final decision and administrative agreement in the frontend phase of project such as Crossrail in London,UK and RandastaRail in Netherland. Figure 2-2 shows the duration of the front-end phase of some MTPs. During this long time of front-end phase many essential factors of project may change and effect on project 


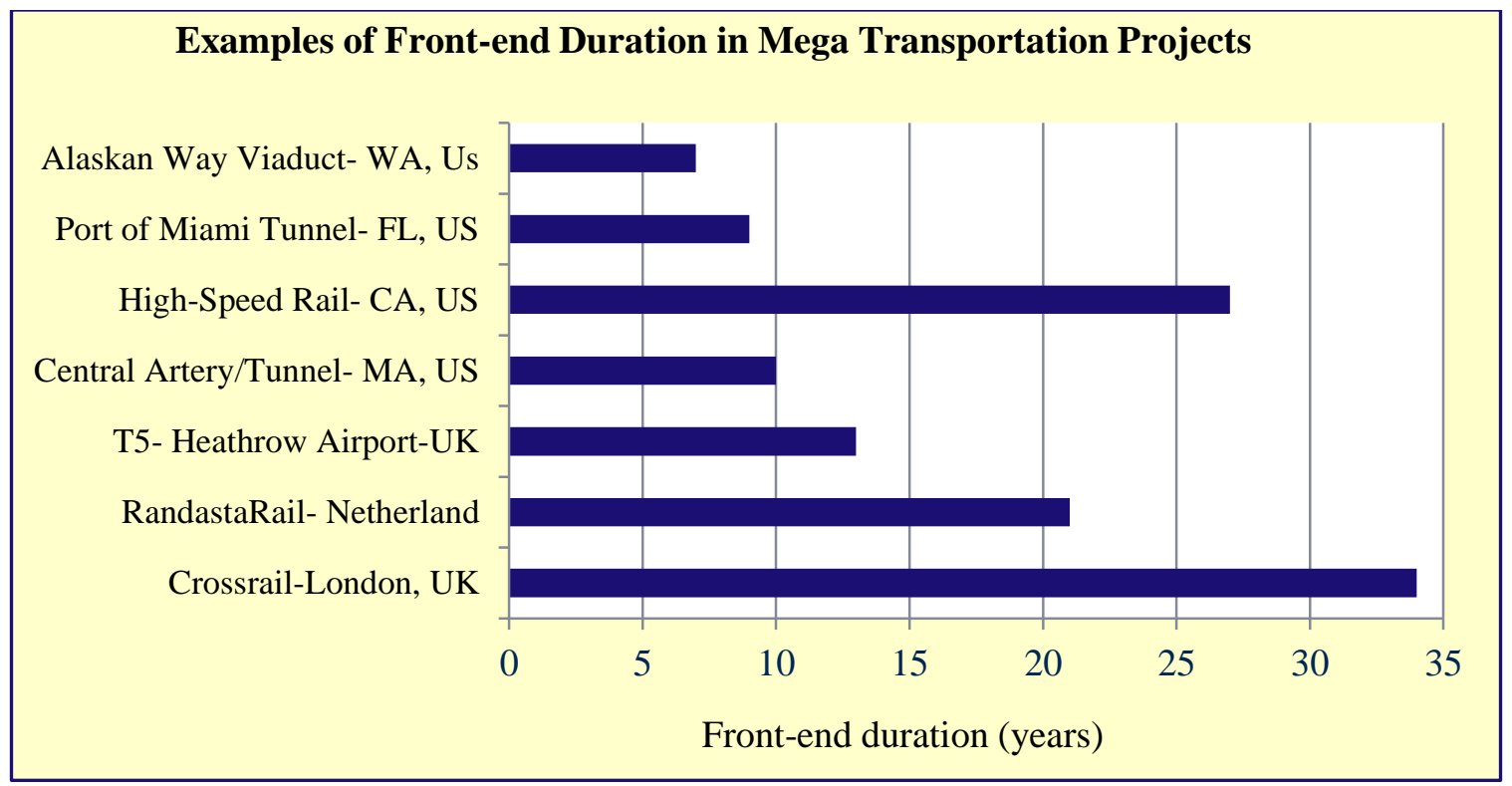

Figure 2-2: Front-end phase duration of various Major Transportation Projects

A CII study among 1081 revealed that paying more attention to front-end phase in projects encompasses several benefits such as: $\$ 3$ to $\$ 10$ payback for each dollar spent in this phase; 6 to $25 \%$ cost savings in the project, and 6 to $39 \%$ schedule reduction which shows the importance of this phase (CII 2012).

\subsection{Decision-Making for Alternative Selection in MTPs}

The Decision-making process for alternative selection in MTP embraces several issues, as well as multiple interests, players, and objectives. All MTPs similar to other projects are a solution to an existing problem or need, so at the beginning stage of project evolution several alternative competing proposals are offered by the project team to meet the demand. One major step in front-end phase is to identify which alternative will act more appropriate in that condition. There are many concerns in alternative selection process that should be considered by the decision-makers. Commonly used methods and important factors in project selection and assorted qualitative and quantitative project selection models has been discussed intensively by researchers such as Khraibani et al. 
(2016), Salling and Banister (2009) Day (2006), Berechman and Paaswell (2005), Vreeker et al. (2002), Vickerman (2000), Morisugi (2000), and Lee (2000).

Evaluating the expected impacts of alternative decisions and policies on the performance of existing or future transportation systems is required before taking the decision. Such impacts include economics (such as quantified benefits and costs); economic development (such as job increases); environmental or ecological impacts (such as air, water, or noise pollution, community effects, and land-use shifts); and technical impacts (such as changes in facility condition, vulnerability and longevity, network mobility and accessibility, and facility and user safety and security). Based on the project size and type, scope and various involved disciplines such as operation research, engineering and environmental science, and economics different methodologies for alternative project evaluation will be applied. This assessment should be broad to include both project level and network level impacts of each alternative project (Sinha and Labi 2011).

Szyliowicz (2003) grouped all general approaches to planning and decisionmaking into two major categories: rational actor models and strategic (or adaptive) models. In the first category, decision-making process traditionally follows a systematic and step-by step predefined stages to reach the solution for a problem. These approaches are often rigid and thus, become impractical in maximizing the benefits and minimizing the costs. While the later group of models are fundamentally flexible, and require monitoring feedback, and make adjustments through a process of trial and error learning. He stressed that adaptive models will be more applicable and successful in the projects that need public participation(Szyliowicz 2003). 
One of the measures to assess different alternatives is economic efficiency that compares the combined monetary cost and benefit impact of each alternative and is

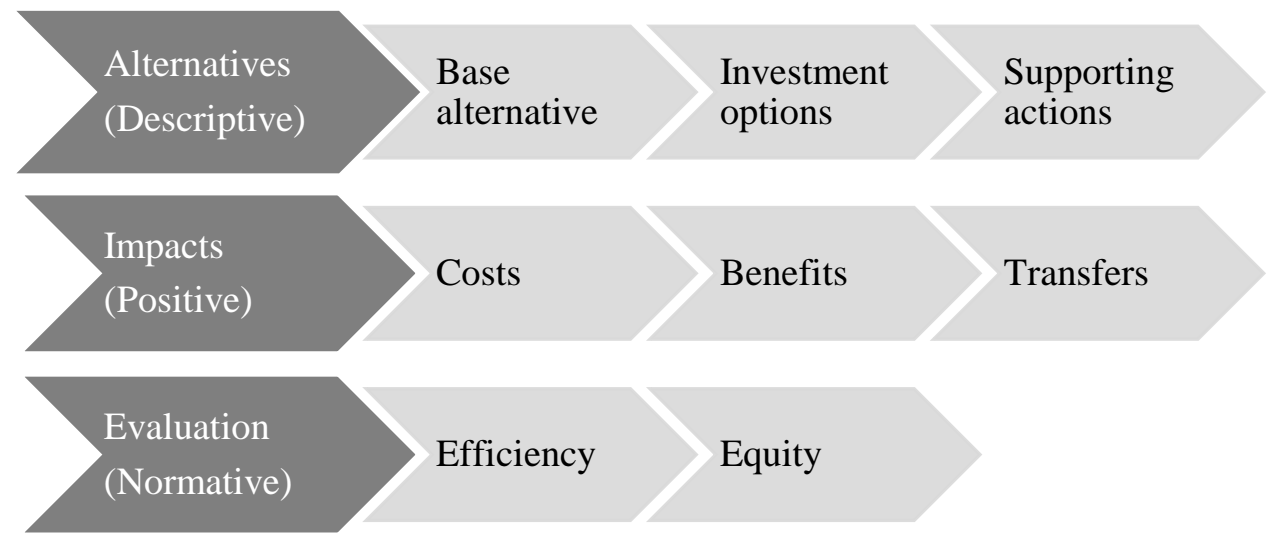

Figure 2-3: Major components of Cost-Benefit Analysis (Lee 2000)

derived from economic analysis. Economic analysis evaluates the efficiency of an investment from monetary viewpoint and used as a decision-making tool by calculating monetized costs and benefits of different alternatives. The timing of the costs and benefits are also important in economic analysis as well as their amount. This method is often referred to as Cost-Benefit Analysis (CBA) and was applied broadly in different countries during past decades not only at the beginning stage of a project, but also in design, construction, maintenance and operation stages (Sinha and Labi 2011). Thus, CBA is a systematic enumeration evaluation technique that works by expressing both the costs and benefits of a set of possible alternatives. It evaluates all the relevant direct and indirect costs and revenues derived from the alternatives. For example, in environmental assessment it evaluates the benefits of protecting the environment and natural resources against the costs associated with environmental damage and control mechanisms produced by the alternatives. The CBA became as increasingly practical method for evaluation after 1950s particularly for public projects. But the essential problem of 
applying CBA is determination of consistent and reliable monetary values for project outcomes (Vreeker et al. 2002)

The main project appraisal approach used in the U.S. federal agencies is BenefitCost (BC) framework (Lee 2000). Its technical process can be divided into alternatives, impacts, and evaluation phases as shown in the Figure 2-3. In Alternatives phase, the essential comparison base is "base alternative" or "do nothing/current condition". It is the most persuasive while efficient usage of existing facility without substantial more investment. Other proposed alternatives require capital investment and usually include different modes of transportation, different locations, facility type or size. All the proposed alternatives should be compared with the base alternative. To compare these alternatives, all their impacts are classified into costs, benefits, and transfers in this method. The main part of impacts are transfers which are the part that assumed the society will be unaffected in the aggregate and mostly individuals will be influenced. The difference between total costs and benefits is all that matters in this method. Some examples of costs and benefits include: Saving time; reducing user, agency, and external costs; improving safety; improving quality; increasing consumer surplus and etc. (Lee 2000). An important issue in carrying out this method is to identify correctly the benefits and costs associated with each project alternative to prevent over/underestimating of impacts. The selection of these impacts also can be influenced by the preference of the funding agency and the available data (Sinha and Labi 2011). There are several published guidance for the U.S. federal and transportation agencies to apply this method from early 1990s. 
Lee (2000) also studied other evaluation methods that are barely used and mostly categorized as scoring-and-weighting methods such as multi-objective programming, goals-achievement, cost-effectiveness, sufficiency ratings, and process-oriented techniques (e.g. Delphi) (Lee 2000).

Despite its widely use and explicit evaluation manner with a clear final choice, CBA has intrinsic shortcomings and practical limitations such as lack of considering the uncertainty, difficulty of obtaining accurate information specially in the early stages of the projects, and strong impact of discount rate or distributional equity(Hall 2006; Vreeker et al. 2002). To address these shortcomings, several researches have been done in the in the past decade. Some of these enhanced models are discussed in the following paragraphs.

In general, the alternative evaluation approaches in decision-making can be categorized in four groups: i) a monetary decision approach, based on Cost-Benefit or cost-effectiveness principles; ii) a utility theory approach, based on prior ranking of the decision-makers' preferences using multi-criteria analysis; iii) a learning approach, based on a sequential (interactive or cyclical) articulation of the decision-maker's views; iv)a collective decision approach, based on multi-person bargaining, negotiation or voting procedures .

Murisogi (2000) examined the system and guidelines for transportation project evaluation in Japan. The studied assessment methods applied a kind of Multi-Criteria Analysis (MCA) in addition to Benefit Cost Analysis (CBA) along with quantitative and/or qualitative evaluation. It included different considerations such as regional economic impacts, global and local environmental impacts, contribution in reaching 
minimum living standards and etc. In this method CBA used as a threshold for accepting the project as a candidate while MCA is applied to rank the priority of projects (Morisugi 2000).

Vickerman (2000) did the same study about project appraisal methodologies in the U.K. simultaneous with Lee (2000) and Murisogi (2000). His studies showed that CBA was used both to evaluate and rank road projects, however for rail projects a simplified procedure was applied rather than CBA. The impact of each alternative project was calculated based on a accurate transportation model, and benefits included time saving and reduction in accident (Vickerman 2000). The U.K government also developed a New Approach to Appraisal (NATA), which is a framework to examine the prioritization of road projects. It included the elements, which were not considered in CBA. Five main criteria were: environmental impact, safety, economy, accessibility and integration. Each criterion had both qualitative and quantitative elements.

Table 2-1 shows the summary of assessed criteria in different evaluation methods used in different countries.

Other studies conducted in project evaluation and selection for fields other than construction. For example, Day (2006) developed a decision support system (DSS) for industrial project assessment using analytic hierarchy process (AHP), which is a multipleattribute decision-making technique. The project stakeholders had been involved in model development. This model considers uncertainties for project financial analysis while selecting the optimal project for investment and suggests mitigation measures (Dey 2006). He also discussed various operational research methods utilized in former project evaluation and selection and listed them as: Utility function, Goal programming, Fuzzy 
theory, 0-1 mathematical modeling, and 0-1 integer linear programming model (Dey 2006).

Table 2-1: Transportation project assessment criteria in different methods

\begin{tabular}{|c|c|c|c|c|c|}
\hline Criteria & $\begin{array}{l}\text { CBA } \\
\text { (US) }\end{array}$ & $\begin{array}{l}\text { CBA } \\
\text { (UK) }\end{array}$ & $\begin{array}{l}\text { NATA } \\
\text { (UK) }\end{array}$ & EU & Japan \\
\hline \multicolumn{6}{|l|}{ Environmental impact } \\
\hline Noise & & & $\checkmark$ & $\checkmark$ & $\checkmark$ \\
\hline Local Air Quality & & & $\checkmark$ & $\checkmark$ & $\checkmark$ \\
\hline Global Air Quality & & & & $\checkmark$ & \\
\hline Landscape & & & $\checkmark$ & $\checkmark$ & $\checkmark$ \\
\hline Biodiversity & & & $\checkmark$ & & \\
\hline Heritage & & & $\checkmark$ & & \\
\hline Water Pollution & & & $\checkmark$ & $\checkmark$ & \\
\hline Severance & & & & $\checkmark$ & $\checkmark$ \\
\hline Global Warming & & & & $\checkmark$ & $\checkmark$ \\
\hline Vibration & & & & $\checkmark$ & \\
\hline Resource Consumption & & & & $\checkmark$ & \\
\hline Visual Intrusion & & & & $\checkmark$ & \\
\hline Safety & $\checkmark$ & $\checkmark$ & $\checkmark$ & $\checkmark$ & $\checkmark$ \\
\hline Economy & & & & & \\
\hline Journey Times And Vehicle Operating & $\checkmark$ & $\checkmark$ & $\checkmark$ & $\checkmark$ & $\checkmark$ \\
\hline Costs & & & $\checkmark$ & & \\
\hline Journey Time Reliability & & & $\checkmark$ & $\checkmark$ & \\
\hline Land Use & & & & $\checkmark$ & \\
\hline Increase In Employment And Income & & & & $\checkmark$ & \\
\hline \multicolumn{6}{|l|}{ International Traffic } \\
\hline \multicolumn{6}{|l|}{ Accessibility } \\
\hline Access To Public Transport & & & $\checkmark$ & & \\
\hline Community Severance & & & $\checkmark$ & & \\
\hline Pedestrians And Others & & & $\checkmark$ & & \\
\hline Integration & & & $\checkmark$ & & \\
\hline \multicolumn{6}{|l|}{ Road users } \\
\hline Reduction Of Traffic Accident & & & & & $\checkmark$ \\
\hline Enhancement Of Driving Comfort & & & & & $\checkmark$ \\
\hline Enhancement Of Safety And Comfort On & & & & & $\checkmark$ \\
\hline \multicolumn{6}{|l|}{ Sidewalks } \\
\hline \multicolumn{6}{|l|}{ Passenger Cost Savings } \\
\hline \multicolumn{6}{|l|}{ Public sector } \\
\hline Local And National Tax & & & & $\checkmark$ & $\checkmark$ \\
\hline Toll Revenue & & & & $\checkmark$ & $\checkmark$ \\
\hline Savings In Public Service Cost & & & & $\checkmark$ & $\checkmark$ \\
\hline Governmental Subsidy And Investment & & & & $\checkmark$ & $\checkmark$ \\
\hline Service Level & & & & $\checkmark$ & \\
\hline \multicolumn{6}{|l|}{ Capital } \\
\hline Construction And Maintenance Cost & $\checkmark$ & $\checkmark$ & $\checkmark$ & $\checkmark$ & $\checkmark$ \\
\hline Disruption Cost & & & & $\checkmark$ & \\
\hline Land And Property Cost & & & & $\checkmark$ & \\
\hline
\end{tabular}


More recent studies focused on combining CBA with other methods to meet the shortages of this method. For example, Ambrasaite et al. (2011) developed a decision support system, Composite Modeling Assessment (COSIMA), which combined CostBenefit Analysis and multi criteria decision analysis (MCDA) for transport infrastructure appraisal. This method involved both economic and strategic impacts. Since MCDA approach has limitations regarding subjective, they also applied a Monte Carlo simulation to reach the weightings in MCDA-part through a case study of a railroad project connecting Baltic and Poland (Ambrasaite et al. 2011).

There are plenty of studies discussed complexity in major transportation projects and have tried to overcome this issue. Giezen et al (2014) attempted to develop and illustrate the concept of adaptive capacity as a tool for analysis, and tried to express the idea through a case study. Two superior sets of uncertainties are identified in any assessment of transport infrastructure projects; the underlying model uncertainties embedded within any traffic or impact model and the uncertainties in any CBA pricing strategy illustrated in terms of the unit prices associated with each of the prior transport related impacts (Salling, 2008).

All the transportation decisions made in the U.S. should follow a set of legislations, which are ruled by government or other agencies to ensure the high performance of transportation system. In this section some of these laws are introduced. These regulations should be considered in project alternative selection process. The alternatives, which don't meet these requirements, will be deleted from the list. 


\subsection{Decision Support Systems for MTP}

In previous sections the alternative evaluation process as one step of the front-end phase of MTPs was discussed in detail. The current section will introduce a number of existing tools developed to address the problems in decision-making process of transportation projects. Much effort has been made to develop advanced decision-aiding methodologies, reliable decision-making procedures, efficient optimization methods and algorithms as well as user-friendly computer tools for transportation. All of these tools can be called as transportation-oriented Decision Support System (DSS).

Based on the researchers' definitions (Zak 2010), transportation-oriented decision support systems are all computer-based tools supporting the decision-making processes in transportation. In this meaning all information management systems, data analysis methods as well as spreadsheets applied to solve transportation decision problems can be classified as transportation-oriented DSSs. Transportation-oriented DSSs are developed mainly to select the most appropriate solution for specific problem, and help the decisionmakers to gather and process the relevant data through the model in different categories of transportation projects. DSSs help the user add value to the system output and perceive a solution rather than providing a direct solution. Every DSS consists of four essential interrelated components: (i) human input, (ii) data describing the problem, (iii) procedures for operating the system, and (iv) computerized system (Zak 2010). The final objective of the current research is to develop a transportation-oriented DSS to utilize in the front-end phase of major transportation projects.

A few DSSs have been found in the literature that investigated the early stage of transportation projects in macro level including: CBA-DK, COSIMA, Simulation- 
Enhanced Approach for ranking MTPs and UNITE-DSS. Table 2-3 shows a list of the existing models with a brief explanation and explored gaps.

The CBA-DK coupled BCA (as a tool to produce single point estimate) with quantitative risk analysis using Monte Carlo simulation to produce interval results (Salling and Banister 2009). The model considered investment costs and travel timesaving, however it does not include the impacts of non-monetary factors, which should be considered in the tourism effect and accessibility effect assessment. The wider and long-range impacts and strategic impacts also are not included in this model.

Another similar decision support model has been developed by Barfod et al. (2011) which is called Composite Model for Assessment (COSIMA) and combined BCA with multi-criteria decision analysis (MCDA) for the assessment of economic as well as strategic impacts within transport projects (Barfod et al. 2011). This model like CBA-DK did not account for the role of different stakeholders of the project.

UNITE-DSS decision support model (Salling 2013) is developed to combine both in single aggregated estimates such as the Benefit Cost Ratios (BCRs), and interval results by accumulated probability curves (or accumulated descending graphs ADG). This study investigated a method to scrutinize the feasibility risk in the time of transportation infrastructure projects assessment. By adding to the conventional CBA through the adoption of a quantitative risk analysis, the probabilities of occurrence of particular risk factors can be incorporated, and decision-makers and analysts can make use of their expertise. The technique used is Monte Carlo simulation, which involves a random sampling method (in this case in terms of a Latin Hypercube sampling approach) concerning each different probability distribution selected for the actual model set-up. 
The selection of the most appropriate probability distribution has been a major task of the research where several distributions have been tested in terms of their suitability in previous Salling's studies. Salling (2008) proved that an Erlang distribution is representing the inaccuracies as concerns construction cost estimates and a Beta-PERT (Program and Evaluation Review Technique) or Normal distribution representing the inaccuracies as concerns the demand forecast estimation (Salling 2013). All the abovementioned existing DSSs have strengths and weaknesses. Particularly they have shortcomings in the front-end phase of major transportation projects. The next section provides a gap analysis on reviewed DSSs. However, the main body of the created framework in this research has been developed based on the strength of existing models. 
Table 2-2: Existing Decision Support Systems for MTPs

Existing Models Description Gap

Analytic

Hierarchy

Process (AHP)

(Saaty 1990)
AHP-technique (set the preferences considering pair wise comparisons of the alternatives within each of the criteria using an intensity scale of importance from 1 to 9)
It does not account for the variations in rankings for setting these priorities. Nor does it adequately consider the compatibility with the proposed projects and the national policies in transport infrastructure development.
Multi-criteria

Decision Support

Methodology for

Evaluating

Airport

Expansion Plans

(Vreeker et al. 2002)
Regime Analysis (an advanced pairwise comparison method)+ AHP + Flag Model (based on critical threshold value analysis).
It is a suitable model to evaluate the qualitative measures such as spatialeconomic and environmentaleconomic policy issues.

It doesn't consider the stakeholders' role in the decision-making process.
Simulation-

Enhanced Approach for ranking MTPs

(Su et al. 2006)
The revised AHP method (expands the matrix of the attributes and impacts) + Monte Carlo simulation analysis
The decision-makers' preferences are not clear and it is complicated to follow.

It does not include the determination of non-monetary impacts, which are vital in the

CBA-DK

(Salling and

Banister, 2009)
$\mathrm{CBA}+$ quantitative risk analysis using Monte Carlo appraisal of the tourism effect and accessibility effect. It also does not consider the wider and long-range impacts and strategic impacts. Furthermore, uncertainty needs more investigation.

Composite model for assessment (COSIMA)

(Barfod et al., 2011)
$\mathrm{CBA}+$ multi-criteria decision analysis (MCDA) for the assessment of economic as well as strategic impacts
It does not account for the role of different stakeholders of the project.
UNITE-DSS decision support model (Salling, 2013)
Benefit Cost Ratios (BCRs) + accumulated probability curves (or accumulated descending graphs ADG).
It requires considerable amount of historic data to create these probability curves. 


\subsection{Gap in Existing Methods of Front-end Phase Decision Making in MTPs}

As described above, a few disadvantages have been identified in the reviewed models and methodologies particularly in deploying them for major transportation projects. First, the predominant method used in MTPs decision making the conventional CBA method, and it calculates a deterministic single point evaluation criteria and its major shortcomings is that uncertainties can only be handled by sensitivity tests in terms of worst and best case scenario. Moreover, tremendous effort in collecting accurate data is required for all the CBA-based methods that makes it almost impossible especially in the early stages of the projects, which encompass large ambiguity. Secondly, some of them need considerable amount of historic data to draw the probability curves in the model, which is often impossible in large-scale projects (They are usually unique and innovative). Thirdly, the wider economic impacts are rarely considered in these models, while it is essential in major transportation projects. Finally, the important role of multi stakeholders is dismissed in most of these models. However, intrinsically numerous stakeholders, sometimes with contrasting interests, are engaged in MTPs.

Additionally, this research attempts to create a framework, which addresses parts of main pitfalls identified by Priemus (2010) in decision-making process of MTPs including, but not limited to: Insufficient problem analysis; Inadequate alternatives; Absence of a well-organized functional program; Unclear project scope; Improper approach to increase quality and innovation along with cost reduction; Misinformation related to the interaction between players; and Lack of explicit or implicit prioritization method for major projects. 
Thus a new method is needed to address all these shortcomings and be adapted to the major transportation projects. To provide a stochastic method that represent the uncertainty of decision-making, a Bayesian Belief method has been used. The following section gives a brief introduction of its basic.

\subsection{Bayesian Belief Network}

Bayesian belief networks is one of the methods that enable researchers to construct tools to perform probabilistic inference to support belief updating and decision making under uncertainty, while acquire knowledge from data/experience and solve problems efficiently and respond to new situations. Bayesian networks and influence diagrams are ideal knowledge representations for use in many situations involving belief update and decision making under uncertainty. These models are often characterized as normative expert systems as they provide model-based domain descriptions, where the model is reflecting properties of the problem domain (rather than the domain expert) and probability calculus is used as the calculus for uncertainty (Kjaerulff and Madsen 2008).

A Bayesian network model representation of a problem domain can be used as the basis for performing inference and analysis about the domain. Decision options and utilities associated with these options can be incorporated explicitly into the model, in which case the model becomes an influence diagram, capable of computing expected utilities of all decision options given the information known at the time of decision. Bayesian networks and influence diagrams are applicable for a large range of domain areas with inherent uncertainty. One of these domains is construction industry. In this research Bayesian network model has been used to develop the final framework. Following part of this section explains more details about Bayesian network model. 
A Bayesian network is a formal statistic-modeling tool that can be described briefly as an acyclic directed graph (DAG), which defines a factorization of a joint probability distribution over the variables that are represented by the nodes of the DAG, where the factorization is given by the directed links of the DAG.

The nodes of the DAG are usually illustrated as circles or ovals and have a finite set of states. The edges, also called arcs or links, represent the probabilistic causal dependence among the variables, which is described probabilistically in a conditional probability table. The nodes with edges directed into them are called "child" nodes and the nodes at the tail of the edge are called "parent" nodes (if there is an edge from node A (the parent node) to another node $G$ (the child node) shows that node $A$ has an influence on node G. While the DAG depicts the qualitative part of causal reasoning in a $\mathrm{BBN}$, the corresponding states give the quantitative part, consisting of a Conditional Probabilistic Table (CPT). A conditional probability indicates the likelihood of a state of a variable that is dependent on the state of another variable. Bayes' theorem is used to recalculate the belief about the state of a node depending on the evidence introduced for another node. A node that has no incoming edges is said to have no parents, e.g., nodes A and E in Figure 2-4. Such nodes can be described probabilistically by a prior (or unconditional) probability distribution. A node can represent any kind of variable such as an observed measurement, a parameter, a latent variable, or a hypothesis. The Bay's theorem says that a Bayesian Network is a representation of the joint distribution over all the variables shown in the DAG and the marginal and the conditional probabilities can be computed for each node of the network (Bayraktar and Hastak 2009; Kjaerulff and Madsen 2008; Trucco et al. 2008). 


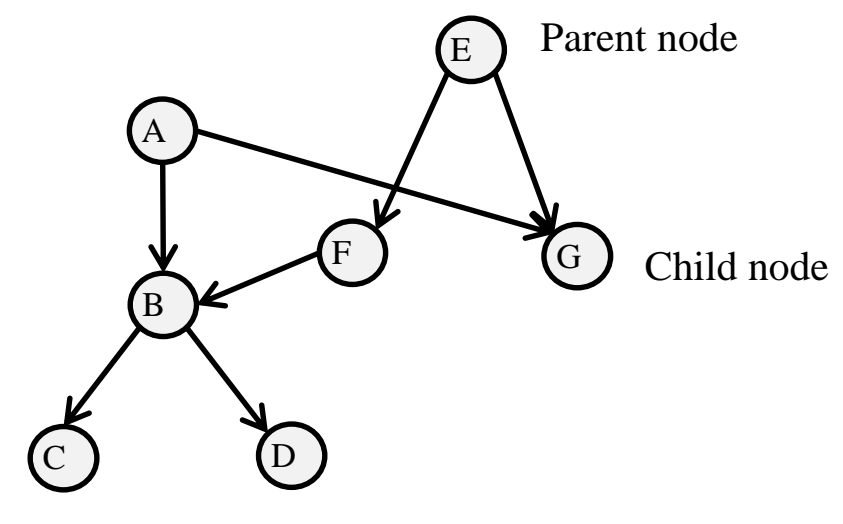

Figure 2-4: Example Bayesian Belief Network

Despite its extensive application in medicine or computer science, the Bayesian belief networks have been applied in a few construction researches. McCabe et al. (1998) used belief networks to provide diagnostic functionality to the performance analysis of construction operations. McCabe (2001) developed an approach based on the Bayesian belief networks for engineering applications. Attoh-Okine and Gibbons (2001) utilized belief network for decision-making about redevelopment of brownfield infrastructures, which faces a complex interrelation among technical issues, liability issues, financial issues, community concerns, and future land-use concerns(Attoh-Okine and Gibbons 2001). Attoh-Okine (2002) also introduced a method using belief networks to make inferences in highway construction costs. Nasir et al. (2003) applied Bayesian belief network to develop a construction schedule risk model. Bayraktar and Hastak (2009) developed a decision support framework to select the optimal contracting strategy in highway work zone projects using Bayesian belief network (Bayraktar and Hastak 2009). Bayesian belief network has been also utilized for risk assessment by some researches. 
Troccu et al.(2008) used Bayesian belief network to integrate human and organizational factors into risk analysis. The model was developed for the maritime transport system, by taking into account its different actors (i.e., ship-owner, shipyard, port and regulator) and their mutual influences (Trucco et al. 2008). Lee et al. (2009) also presented a scheme for large engineering project (i.e. Korean shipbuilding industry in that research) risk management using Bayesian belief network (Lee et al. 2009)

\subsection{Multi-criteria Analysis Models}

Decision-making under information uncertainty and conflict management of numerous stakeholders is commonly a critical issue. Many conflicting views may emerge in evaluating MTP alternatives. Approaches like multi criteria analysis may serve as a meaningful evaluation vehicle for taking explicitly account of such conflicts regarding the foreseeable impacts of a plan. Multi criteria analysis may then be helpful in taking into account such conflicting issues by considering priority schemes or weights as an ingredient in an evaluation analysis for investment projects. Of course, this will not always lead to a unique final solution, but the structure and consequences of conflicts among decision-makers can be made more explicit, so that also the range of politically feasible alternatives can be analyzed in greater detail. (Vreeker et al. 2002).

In general, the decision basis for a MCDM framework is composed of the components that represent states, relationships, alternatives, preferences, and interrelations between them. A combination of Bayesian Network method and MCDA approach is used by Watthayu and Peng (2004) to capture the above mentioned uncertain interrelations and provides mechanisms for decision-making based on this representation (Watthayu and Peng 2004). 
In multi criteria decision making, a key step is the explicit or implicit assignment of relative weights to each performance criterion to reflect its importance compared to other criteria; for example, to what extent is safety improvement more important than travel-time reduction, or increased economic development, and so on? Different methods have been used in the literature to establish the weights: (1) equal weighting, (2) direct weighting, (3) regression-based observer-derived weighting, (4) the Delphi approach, (5) the gamble method, (6) pairwise comparison, and (7) value swinging (Watthayu and Peng 2004).

One useful method of multi criteria decision-making model is Flag model, which is found well-suited to the parameters of the current research. Nijkamp and Vreeker (2002) presented the Flag Model that is a framework to assess the sustainability of development strategies at a regional level, with a particular view on the treatment of uncertain information. They adopt the view that "sustainability means that the development of an economy has to take place within a set of pre-specified normative constraints or pathways". This framework is based on a systematic multi criteria flag model capable to take into account Critical Threshold Values (CTV). A CTV is defined as "the numerical normative value of a sustainability indicator that ensures a compliance with the carrying capacity of the regional environmental system concerned". They indicated that CTV are based on scientific information and expert opinion, more detail is not given. Exceeding a CTV would impose an unacceptably high cost on the environment. In this method, reference values are not a single value but a band width, defined by $\mathrm{CTV}_{\min }$ and $\mathrm{CTV}_{\max }$, to reflect uncertainty. This band width mirrors the range of CTV values expressed by experts or policy makers. $\mathrm{CTV}_{\min }$ indicates a conservative 
estimate of the threshold, while $\mathrm{CTV}_{\max }$ refers to a maximum allowable value, with $\mathrm{CTV}_{\text {int }}$ being halfway between $\mathrm{CTV}_{\min }$ and $\mathrm{CTV}_{\max }$. Color "flags" are attributed to indicator values: green (no reason for concern) for values below $\mathrm{CTV}_{\min }$; yellow (be alert) for values between $\mathrm{CTV}_{\text {min }}$ and $\mathrm{CTV}_{\text {int }}$; red (reverse trends) for values between $\mathrm{CTV}_{\text {int }}$ and $\mathrm{CTV}_{\max }$; and black (stop immediately further growth) for values above $\mathrm{CTV}_{\max }$. Three development scenarios for the southern peninsular region of Thailand were compared using eighteen indicators summarizing social, economic and environmental sustainability (Vreeker et al. 2002)

\subsection{Monte Carlo Simulation}

Sequential Monte Carlo (SMC) is a set of methods allowing us to approximate virtually any sequence of probability distributions. SMC are very popular in physics where they are used to compute eigenvalues of positive operators, the solution of PDEs/integral equations or simulate polymers. SMC is also applied vastly in construction management to provide stochastic solutions in scheduling and planning.

It is a computerized tool for modeling a stochastic process where the input data are randomly determined by certain statistical distributions. In such a simulation, the computer generates large sets of outputs after running a large number of iterations with random inputs. These outputs are then statistically analyzed to measure their uncertainties and risks. The major parts of a Monte Carlo simulation method involve a probability distribution function, a random number generator, and a sampling rule (Bayraktar and Hastak 2009).

Monte Carlo simulation was developed in the 1940s and became available under the help of personal computers and associated software, e.g. Primavera Risk, Primavera 
Crystal Ball, @RISK for Projects and so on. Monte Carlo has been involving in many industrial, scientific, logistical and social fields in many years. Recently project managers have used Monte Carlo to simulate the project completion date and the project cost estimate. Through computing the Critical Path Method schedule many times, Monte Carlo simulation can determine the project completion date on the basis all possible combinations of the uncertain activity durations. In Monte Carlo simulation, the project model is computed many times (iterated) with the input values (e.g., activity durations) chosen at random for each iteration from the probability distributions of these variables. Monte Carlo simulation follows certain steps:

1. Creation of the quantitative model of the form of $y=f(x 1, x 2, \ldots, x n)$,

2. Selection of the random variables xi1, xi2, .., xin,

3. Evaluation of the model and output storage in yi,

4. Repeat steps (b) and (c) as many times needed (for $\mathrm{i}=1$ to $\mathrm{k}$ ),

5. Analysis of results.

Su et al. (2006) suggested Monte Carlo simulation to use in decision-making models to overcome the inherent deterministic aspect of benefit- cost analysis method. As explained in the previous sections, they combined BCA with Monte Carlo simulation to get more reliable alternative assessment tool (Su et al. 2006).

\subsection{Chapter Summary}

This chapter provided an extensive literature review of the decision making process in the front-end phase of major transportation projects, as well as existing decision support systems and gap analysis in the existing approaches. It also overviewed the basic concepts of Bayesian belief network and multi-criteria decision making 
methods and theories that will be utilized for developing the created framework in the course of this research. The following chapter will describe in detail the current state-ofthe-practice of the decision-making procedure in the U.S. based on the findings and analysis of a questionnaire survey conducted at DOT's and also reviewing the current regulations and published guidelines. 


\section{CHAPTER 3. STATE-OF-THE PRACTICE}

\subsection{Introduction}

The objective of this chapter is to provide an overview of the process of decisionmaking in major transportation projects in the relevant agencies of the U.S. and present methods and frameworks through which planning experts achieve the prioritized alternative as well as the assessing indicators and factors. Transportation decision-making performs at several levels of government in the U.S. Transportation planners work across all modes of transportation, and with environmental resource agencies, tribes, and interested parties as defined by law.

State Departments of Transportation (State DOTs), Metropolitan Planning Organizations (MPOs), and transit agencies develop the transportation planning in different levels and then, USDOT surface transportation agencies reviews these plans MPOs are organizations that carry out transportation planning at the regional level. Any urbanized area with a population of more than 50,000 has an MPO (USDOT 2015). The policy at MPO is set by a board of local elected officials and includes long-range plans and short-range programs of future transportation improvements. Several stakeholder groups, such as nonprofit, community-based, and environmental organizations as well as general public, provide input for MPO's policies, plans, and overall program direction. There are other agencies such as tribal governments, local governments, transit agencies and Regional Transportation Planning Organizations (consist of local governments outside of metropolitan areas) that come together and coordinate with State DOTs in planning process (USDOT 2015). 
Highlighted points of transportation planning process through DOTs extracted from USDOT website are summarized as follows:

- One of the major metrics in project prioritization process in DOTs and MPOs is commonly an effective project performance with meaningful, measurable and monitorable performance measures.

- "There is no one size fits all approach to project prioritization. Each MPO must work with regional stakeholders to develop a prioritization process that addresses the region's specific goals, resources, and needs. Participating MPOs use different approaches and criteria to evaluate, prioritize and select projects for long-range plan. Some prioritization processes are more detailed and technical, while others reflect broader policy priorities.”

- Most of the transportation agencies conduct some type of benefit-cost analysis in order to prioritize projects. To develop a meaningful prioritization process it is better to use a mix of quantitative and qualitative tools and analysis. In some types of projects, it can be difficult to accurately measure their costs and benefits, therefore quantitative analysis may not score them well. It is important to build qualitative factors into the prioritization process by providing supplementary information for projects whose benefits and costs may be difficult to quantify.

- "Weighting of project prioritization criteria should be left to the discretion of each agency's decision-making boards".

- Transportation agencies often tend to focus their prioritization efforts on projects that add new capacity and on funding sources that they have direct decisionmaking authority over, especially on regionally significant projects that add new 
highway or transit capacity in the region. There is also tendency in prioritization analysis for funding sources that these agencies have discretion over, such as Federal Congestion Mitigation and Air Quality funds and Surface Transportation Program.

- Preservation and maintenance of the existing system is a focus for all transportation agencies, but these areas are typically funded on a programmatic basis, rather than as individual projects.

- "Transportation modeling is extremely important for providing guidance on the anticipated outcomes of investments." (USDOT 2015).

\subsection{Legislations and procedures for decision making in major transportation projects}

Before any project can move forward to the construction phase, FHWA and FTA may address and assess compliance with more than 40 laws related to safety and the environment. These laws cover social, economic, and environmental (SEE) concerns ranging from community cohesion to the impact on threatened and endangered species. In order to successfully complete this detailed process, FHWA and FTA use the NEPA process to evaluate the SEE concerns with each individual project. (USDOT 2015)

The need to considering transportation development planning and public involvement in transportation decisions have been recognized for the first time in 1960s, when the continuous, comprehensive, and cooperative (3C) planning process for metropolitan areas had been established by the Federal Highway Act of 1962 established. Then NEPA was enacted in 1969 to promote the transportation decisions 
environmentally. During 1970s several legislations such as the Clean Air Act of 1970, the Endangered Species Act of 1973, and the Resource Conservation and Recovery Act of 1976 were passed. The legislation passed in 1980s considered accessibility of metropolitan area and their connection to interstate highway system. The Intermodal Surface Transportation Efficiency Act (ISTEA) of 1991mandated having a management system on transportation decision-making process. At the beginning of 21 century, state highway agencies were asked to have effective environmental consideration process by the Transportation Efficiency Act of the $21^{\text {st }}$ Century (TEA-21). It includes 7 very important acts, which aggregated 16 previous planning factors. It concentrated on economic vitality of the area, safety and security, accessibility and mobility, protecting and enhancing the environment, energy conservation and quality of life, integration and connectivity of transportation system, efficient system management and operation, and preservation of the existing transportation system. The most recent law, The Safe, Accountable, Flexible, Efficient Transportation Equity Act-A Legacy for Users (SAFETEA-LU) of 2005, contained imperatives for environmental stewardship in planning of highway and multimodal transportation projects (Sinha and Labi 2011).

“The National Environmental Policy Act (NEPA)" process is a broadly used method to assess environmental impact of project alternatives. NEPA process consists of three level of analysis: categorical exclusion determination; preparation of an environmental assessment/finding of no significant impact (EA/FONSI); and preparation of an environmental impact statement (EIS). In Categorical Exclusion level if the project meets certain criteria, which established by federal agencies, it will be determined as having no significant environmental impact and there is no need for further investigation. 
At the second level, EA/FONSI, a written environmental assessment should be determined by federal agency to investigate the environmental impacts of the project and report of finding no significant impact (FONSI) should be issued and it may contain the measures to take for mitigating the potential impacts. If the EA determined significant environmental impacts, the third level of NEPA process will be required which is regarding environmental impact assessment (EIS) report. All public, related federal agencies and other parties engage in preparation of this report (http://www.epa.gov/compliance/basics/nepa.html).

Another method that has been suggested recently in Innovative Program delivery office of FHWA is "Value for Money (VfM)" specifically for Public-Private Partnership projects. Different accounting systems are used by CBA and VfM. The VfM is a financial assessment which considers financial elements only, i.e., cash flows and focuses on costs and revenues; benefits to society (e.g., user benefits from accelerated project delivery or improved performance) not evaluated quantitatively. While CBA is an economic assessment that considers full range of costs and benefits and may include financial elements, but some such elements may not be included, e.g., tolls, taxes, financing. The perspective of CBA is that of society as a whole, however for VfM is that of the procuring agency. Different accounted costs in these two methods are compared in Table 3-1

(http://www.fhwa.dot.gov/ipd/pdfs/p3/mdot_p3_webinar_102114/session_5_vfm.pdf).

A list of risk factors identified by FHWA is as follows: Design errors, Change in scope, Delay in permits, Delay in right-of-way acquisition, Construction cost overruns, construction risks, Archeological findings, Delay in relocation of cables and pipes, 
Unknown ground conditions, Hazmat, Security, Major maintenance cost overruns, Snow and ice removal cost overrun, Regular maintenance, Traffic information systems, Incident management, Toll revenue risk, Financing risks, and Force majeure. This research considered the related factors from this list in developing the framework.

Table 3-1: Comparing CBA and VfM

\begin{tabular}{|c|c|c|}
\hline $\begin{array}{l}\text { Accounting for Project Costs } \\
\text { (both CBA and VfM) }\end{array}$ & $\begin{array}{l}\text { Accounting for other social } \\
\text { impacts } \\
\text { (CBA method only) }\end{array}$ & $\begin{array}{l}\text { Accounting for financing } \\
\text { (VfM method only) }\end{array}$ \\
\hline $\begin{array}{l}\text { Capital costs, O\&M costs, } \\
\text { Risk impacts, Transaction } \\
\text { costs: } \\
\text { Defining outputs } \\
\text { Developing contract } \\
\text { Procurement } \\
\text { Design } \\
\text { Obtaining financing } \\
\text { Monitoring and oversight }\end{array}$ & $\begin{array}{l}\text { User benefits: } \\
\text { Travel time savings } \\
\text { Incident/accident cost savings } \\
\text { Vehicle operation cost savings } \\
\text { External costs and benefits: } \\
\text { Emissions (air pollution, GHG) } \\
\text { Noise } \\
\text { Emergency response }\end{array}$ & $\begin{array}{l}\text { Cash flows: } \\
\text { Revenues (taxes, tolls, etc.) } \\
\text { Debt and equity contributions } \\
\text { Interest and dividend payments }\end{array}$ \\
\hline
\end{tabular}

Based on Minnesota Alternatives Analysis Report the public involvement aspect of the Feasibility Report and Alternatives Analysis Study was developed and conducted consistent with the following four goals:

1. Transparency

2. Comprehensive Sharing of Information

3. Adherence to Principles of MnDOT's "Hear Every Voice" Guidance

4. Application of Systematic Development of Informed Consent (SDIC) Methods

To reach these goals, three separate project advisory committees were established, a flow of information exchange was agreed upon, and a decision-making process was developed. 
Screening criteria are as follows: Design criteria, Compatibility with Environmental Considerations, Preservation of Existing Freight Corridor Alternative analysis, and Operation (including average congestion and average speed by train type).

New York state and specifically New York City involve in a large number of transportation mega-projects, each entailing substantial capital investment proposed by many public agencies and private interest groups. City of NY in partnership with a research group proposed a project alternative evaluation method to be conducted through careful definition of the variables used to measure costs and benefits and through an appropriate assessment methodology. The method combined a Cost Benefit Analysis of transportation benefits with Cost Benefit Analysis of economic development benefits to assess the different alternative projects. Then using a Goal Achievement Matrix as a scoring and whitening method, ranking and prioritization of alternatives can be carried out (Berechman and Paaswell 2005).

\subsection{Contract types in transportation projects}

Another important aspect in decision-making process of MTPs is choosing the contract type that the project will be delivered based on it. There are several types for transportation projects but not all of them are allowed to apply in Public-Private Partnerships (PPP) contracts. Contract types for transportation projects include but are not limited to Concessions, Cost-Plus-Time Bidding (A+B), Lane Rental, Warranty, Build-Operate-Transfer (BOT) Projects, and Design-Build-Operate (DBO) Projects are types of public-private partnerships that are output focused. BOT and DBO projects typically involve significant design and construction as well as long-term operations, for 
new build (greenfield) or projects involving significant refurbishment and extension (brownfield).

A Concession, which gives a concessionaire the long term right to use all utility assets conferred on the concessionaire, including responsibility for operations and some investment, is one of the common methods for PPP. The "Cost-plus-time bidding", like "Lane rental" focuses on accelerating construction process and minimize road user impacts during construction. These two methods became legal to be used in federal projects since 1995. In the "Design-build" contract, contractor is allowed maximum flexibility for innovation in the selection of design, materials and construction methods. With design-build procurement, the contracting agency identifies the end result parameters and establishes the design criteria. Although "Warranties" were used in many countries successfully, it was prohibited by federal rules in the US. However FHWA published warranty Final Rule in 1996, which states that "warranty provisions shall be for a specific construction product or feature. Routine maintenance items are still not eligible" (http://www.fhwa.dot.gov/programadmin/contracts/sep_a.cfm).

\subsection{Importance of public participation in decision making process}

At the most general level, public involvement enhances the legitimacy and political acceptability of policies and projects and can diffuse potential conflicts. Moreover, widespread participation by community members and stakeholders can improve the quality of policies and projects by incorporating knowledge and information that would otherwise not be available to planners. Furthermore, the act of participating

yields numerous powerful personal and political benefits. It is a self-actualization process 
that empowers citizens and minimizes feelings of alienation and impotence from the political system (Szyliowicz 2003).

Public Private Partnerships (PPP) have emerged as one of the major approaches for delivering infrastructure projects effectively. A study by Kwak (2009) revealed that PPP could create new and long-term business opportunities with a chance to deliver infrastructure services of higher quality and efficiency. However, these benefits will only be materialized when a PPP project is properly planned and managed and both the public and private sectors work together successfully (Kwak et al. 2009).

\subsection{Questionnaire Survey}

Chapter 2 presented the results of the literature review on the subject matter and it is conducted by reviewing published consultancy reports, documented research, and other publicly available information sources. Following the literature review, to ensure that no other project assessment methods and decision criteria were missed, a comprehensive survey of state Departments of Transportation (DOTs) and other relevant transportation agencies such as MPOs and FHAW was conducted. An online questionnaire was sent to DOTs to collect data from all states. The survey form was divided into two sections, "A. Contact" and "B. Your experience about major transportation projects decision-making process." Under "A. Contact," the respondent was first asked to provide his or her contact information. The fields included the name of the respondent, title/designation, organization, phone number, and e-mail address. Section B was started with descriptive questions about decision-making process and was followed by tabular-format questions about "Decision Indicators" and "Critical Factors" under each "Decision Indicator." The respondents were asked to provide a contact for further information. They were also 
asked to include a link to the relevant website(s) and any supporting documentation and distribute the questionnaire link among the relevant personnel of their organization or other agencies.

Distribution of the online questionnaire started on January 20th, 2015. For DOTs who did not respond, periodic reminders were sent until April 20th, 2015. After two online reminders, calls were placed to the DOTs and other agencies. Finally, a total of 14 acceptable responses were received from the over 120 contacted persons, yielding a response rate of approximately 10\%. The following sections summarize the results obtained. Respondents are from different states in the U.S. including Colorado, Florida, Kentucky, Missouri, New York, North Carolina, Ohio, Tennessee, and Washington D.C.

\subsubsection{Survey Results}

The first question asked about the agency/organization that identified the need for major transportation projects in that area. The answers include: State DOTs, Counties, Metropolitan Planning Offices, Metropolitan Transportation Council, City's Office of Planning, Community and/or private stakeholders (Advocacy Organizations, Business Improvement Districts, etc.), Regional Highway District Offices, Area Development Districts, Public, Local Rural Planning, and Economic Development. Also Maintenance provides a list of needs some of which could be considered major transportation projects.

Next question was asked about the parties/stakeholders who are engaged in the decision-making process of MTPs. The received answers are as follows:

- Public officials and general public from counties;

- Elected officials in MPOs and Transportation Planning Regions (TPRs), Transportation Commission, senior DOT staff, other stakeholders; 
- Metropolitan Area Transit Authority, County's office of planning, environmental agencies, historic preservation office, regional and citywide stakeholder groups with interest in transportation, land use, and the environment, and neighborhood groups that are directly impacted by project;

- Local leaders and elective leaders;

- Project Teams include various personnel depending on the nature of the project. They frequently include internal representation from across the cabinet (Traffic, Maintenance, Construction, Utilities, Right of Way, Design, Structures, etc). Stakeholders are identified early on in the public involvement process. Additionally, some projects include outreach to various resource agencies to determine potential issues or concerns.

- Local, state, county, federal partners (based on USDOT guidance)

In the third question, the respondents were asked to explain how the different alternative solutions for the identified need are developed in the front-end phase of major transportation project. One answer mentioned that alternative scenarios are developed based on the objectives set by stakeholders, which has to meet Federal mandates and guidelines. The other indicated that it is through the transportation planning process with stakeholders and/or through environmental (NEPA) processes. Some other respondents emphasized NEPA process too. They said DOTs follow a pretty standard NEPA process of identifying a purpose and need for a project and then using professional expertise to identify possible alternative solutions. Solutions are often derived from previous studies and planning efforts. At times, stakeholder involvement will result in the generation of new alternatives, but this rarely happens in the front-end phase. Another respondents 
expressed that "a cross section of alternatives which may address the need are developed. Impacts including cost, relocations, property impacts, environmental impacts, how well it addressed the need, etc. are collected for each selected alternative. Alternatives may be dropped at any point during development if impacts become too severe, cost is unacceptable, or other reasons." The other response was through transportation demand modeling.

The next question was about different tools and methods that they used for evaluation and prioritization of project alternatives in MTPs. Following are the received answers:

- NYBPM - New York Best Practice Model (which is a travel forecasting model) is used to generate and test run alternatives projects and compare the outcomes. Also, Post Processing Software for Air Quality (PPS-AQ) is used for air quality analysis.

- The data-driven project evaluation, including economic analysis. Tools and processes continue to be refined and further developed.

- DDOT has begun using Multi-Criteria Assessments (MCAs) on occasion. So far, I have seen these primarily used as part of an inter-agency process when other agencies have a strong interest in (or control over) project outcomes. More often, the evaluation/prioritization process has seemed a bit fuzzier. Multiple criteria are used (e.g., LOS, safety, transit travel time, ridership), but it is not often clear how these criteria are weighed and how they result in prioritization.

- Financial issues 
- Traditionally decision matrices have been used by the project team to derive a consensus on the preferred alternative.

- USDOT Guidance-transportation demand modeling (macro and micro sim), ADT, Truck percent, environmental, planning and zoning, etc

The next question asked whether they consider Risk Assessment at the front-end phase or not, and 4 respondents out of 6 answered yes. But there was no clear answer about what methods they were using.

The following part of the questionnaire contained questions about decisionmaking Decision Indicators and the critical factors under each of those categories. First, a table of main categories of Decision Indicators that had been obtained from the literature review was given to the respondents and they were asked to identify the level of importance for each Decision Indicator. Table 3-2 includes the data obtained for this question. The mean value of responses shows that "Transportation benefits," "Financial issues," "Environmental impacts" and "Project costs" are of primary importance among all other Decision Indicators respectively. The secondary importance level belongs to "Social impacts" and "Economic development". "Technical issues" is at the next level of importance. The least important Decision Indicator in this list is "Political issues". The other information that can be obtained from responses is that "Transportation Benefits" is the only Decision Indicator, which is currently used in the evaluation process by all respondents.

Table 3-2: Importance level of decision-making Decision Indicators

\begin{tabular}{|l|l|l|l|}
\hline Decision Indicators & \multicolumn{2}{|l|}{ Is currently used in evaluation? } & $\begin{array}{l}\text { Importance Level } \\
\text { (Mean value) }\end{array}$ \\
\hline Economic Development & Yes & No & 4.08 \\
Environmental Impacts & 11 & 1 & 4.33 \\
\hline
\end{tabular}




\begin{tabular}{l|l|l|l} 
Social Impacts & 10 & 2 & 4.17 \\
Project Costs & 11 & 1 & 4.33 \\
Project Transportation Benefits & 12 & 0 & 4.67 \\
Technical Issues & 10 & 2 & 3.92 \\
Financial Issues & 10 & 1 & 4.55 \\
Political Issues & 9 & 3 & 3.50
\end{tabular}

Figure 3-1 depicts the assigned importance level for each Decision Indicator by respondents.

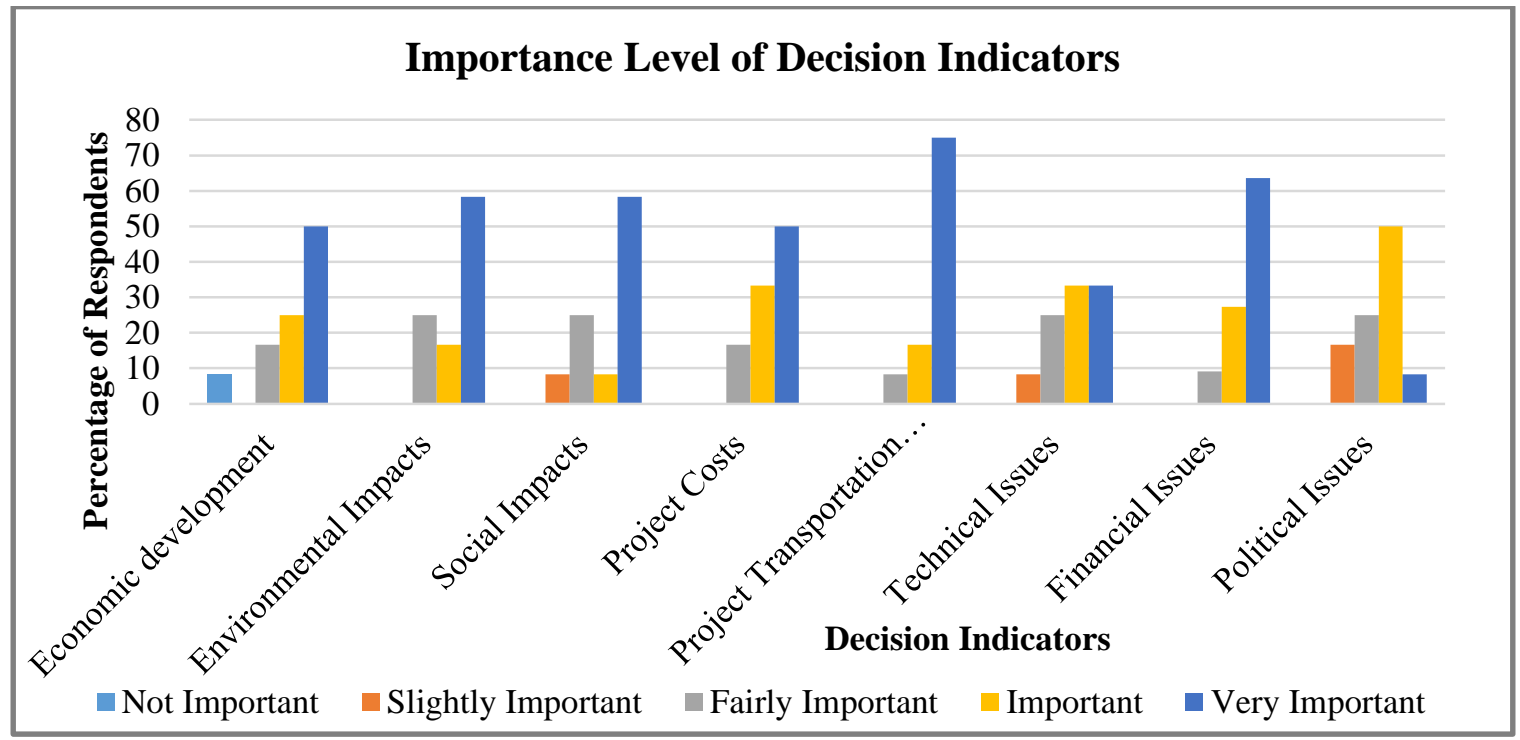

Figure 3-1: Assigned importance level for each Decision Indicator

Respondents also suggested some other indicators as important Decision Indicators that should be added to this list: "Consistency with land-use plans", "Diversity", "Safety", "Access" and "Accessibility of various modes". It was also mentioned that "Economic Development" is a reason a project is funded, but not a reason a particular alternative is selected.

As mentioned above, a list of critical factors were determined for each Decision Indicator through the comprehensive literature review. In the next section of the questionnaire, respondents were asked to evaluate the importance level of the critical factors identified for each Decision Indicators in separate tables. Eleven critical factors 
have been identified for "Economic development" through initial studies. Among them "Increase employment opportunities" is far above the others following with "Business travel time saving" and "Facilitating more efficient trade". "Freight cost saving", "Improve business productivity", and "Improve tourism" are the next important factors. Then "Increase inward investment", "Making a greater labor force available", "Increase incomes of business and property owners" and "Extending geographic markets" got the intermediate level of importance. The least important one is "Improving convention business". Table 3-3 summarizes this information and Figure 3-2 shows the assigned importance level for these critical factors.

Table 3-3: Importance level of critical factors under "Economic development"

\begin{tabular}{|c|c|c|c|}
\hline \multirow{2}{*}{$\begin{array}{l}\text { Critical Factors of "Economic } \\
\text { development" }\end{array}$} & \multicolumn{2}{|c|}{ Is currently used in evaluation? } & \multirow{2}{*}{$\begin{array}{l}\text { Importance Level } \\
\text { (Mean value) }\end{array}$} \\
\hline & Yes & No & \\
\hline Increase employment opportunities & 7 & 3 & 4.50 \\
\hline Business travel time saving & 4 & 5 & 3.89 \\
\hline Facilitating more efficient trade & 4 & 5 & 3.89 \\
\hline Freight cost saving & 4 & 5 & 3.78 \\
\hline Improve business productivity & 4 & 5 & 3.70 \\
\hline Improve tourism & 5 & 4 & 3.56 \\
\hline Increase inward investment & 4 & 5 & 3.40 \\
\hline Making a greater labor force available & 2 & 7 & 3.33 \\
\hline $\begin{array}{l}\text { Increase incomes of business and property } \\
\text { owners }\end{array}$ & 2 & 7 & 3.22 \\
\hline Extending geographic markets & 3 & 6 & 3.22 \\
\hline Improving convention business & 1 & 8 & 2.75 \\
\hline
\end{tabular}




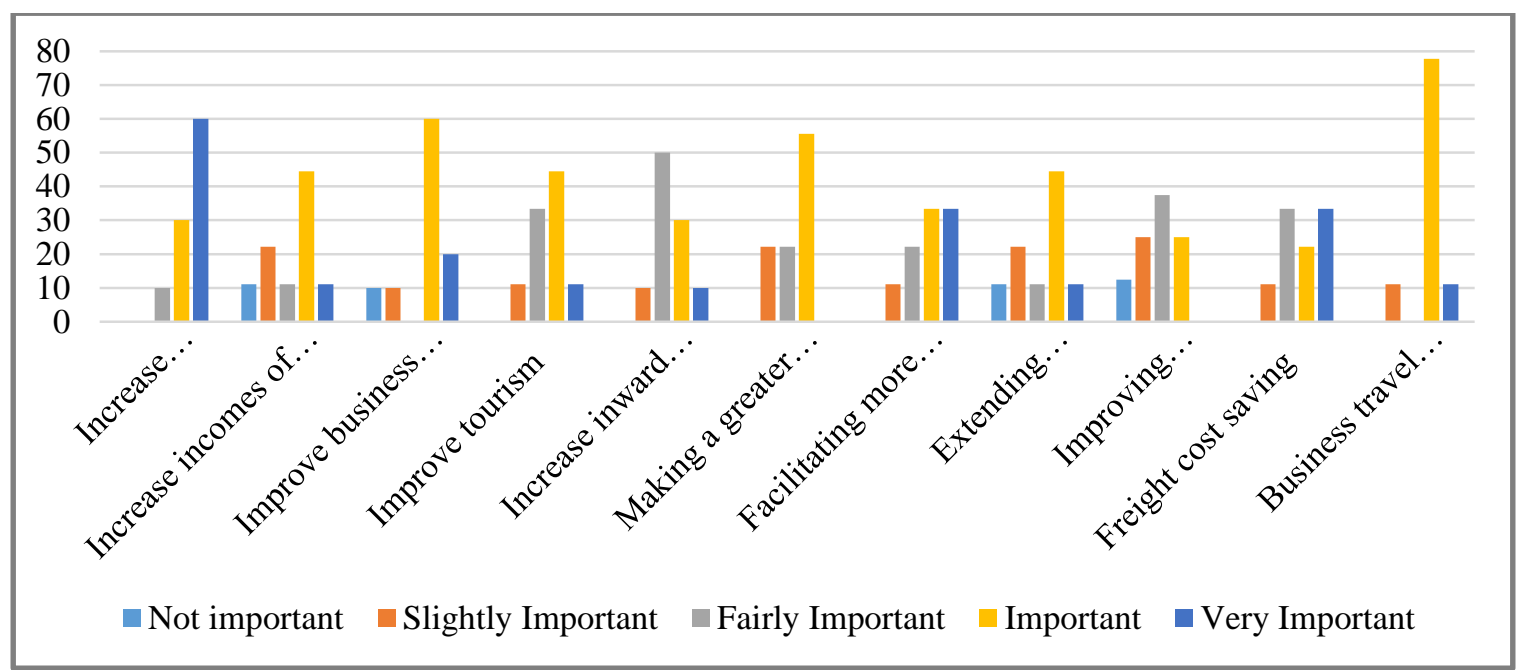

Figure 3-2: Assigned importance level of Critical Factors under "Economic development"

The most important factor filtered for "Social impacts" Decision Indicator was "Savings in lives" which were followed by "Travel time saving" and "Increase employment opportunities". The next important factors were "Relocation of residents or businesses impact", "Connect various industries and communities", "Contribute to development strategies", "Enhance customer service", "Healthier commuters", "Growth in population", and "Offering balanced regional development". The next series of factors with less importance were "Industrial diversification", "Improve tourism" and "Less stressful journeys" respectively.

Table 3-4: Importance level of critical factors under "Social impacts"

\begin{tabular}{|l|l|l|l|}
\hline Critical Factors of "Social impacts" & \multicolumn{2}{|l|}{ Is currently used in evaluation? } & $\begin{array}{l}\text { Importance Level } \\
\text { (Mean value) }\end{array}$ \\
\hline Savings in lives & Yes & 9 & 1 \\
Travel time saving & 9 & 1 & 4.58 \\
Increase employment opportunities & 7 & 2 & 4.50 \\
Relocation of residents or businesses impact & 7 & 3.20 \\
Connect various industries and communities & 7 & 2 & 3.83 \\
Contribute to development strategies & 4 & 5 & 3.73 \\
Enhance customer service & 2 & 6 & 3.70 \\
Healthier commuters & 1 & 8 & 3.63 \\
Growth in population & 6 & 4 & 3.63 \\
Offering balanced regional development & 3 & 6 & 3.60 \\
\hline
\end{tabular}


Industrial diversification

Improve tourism

0

3

Less stressful journeys

3

9

6

3.38

3.11

3.10

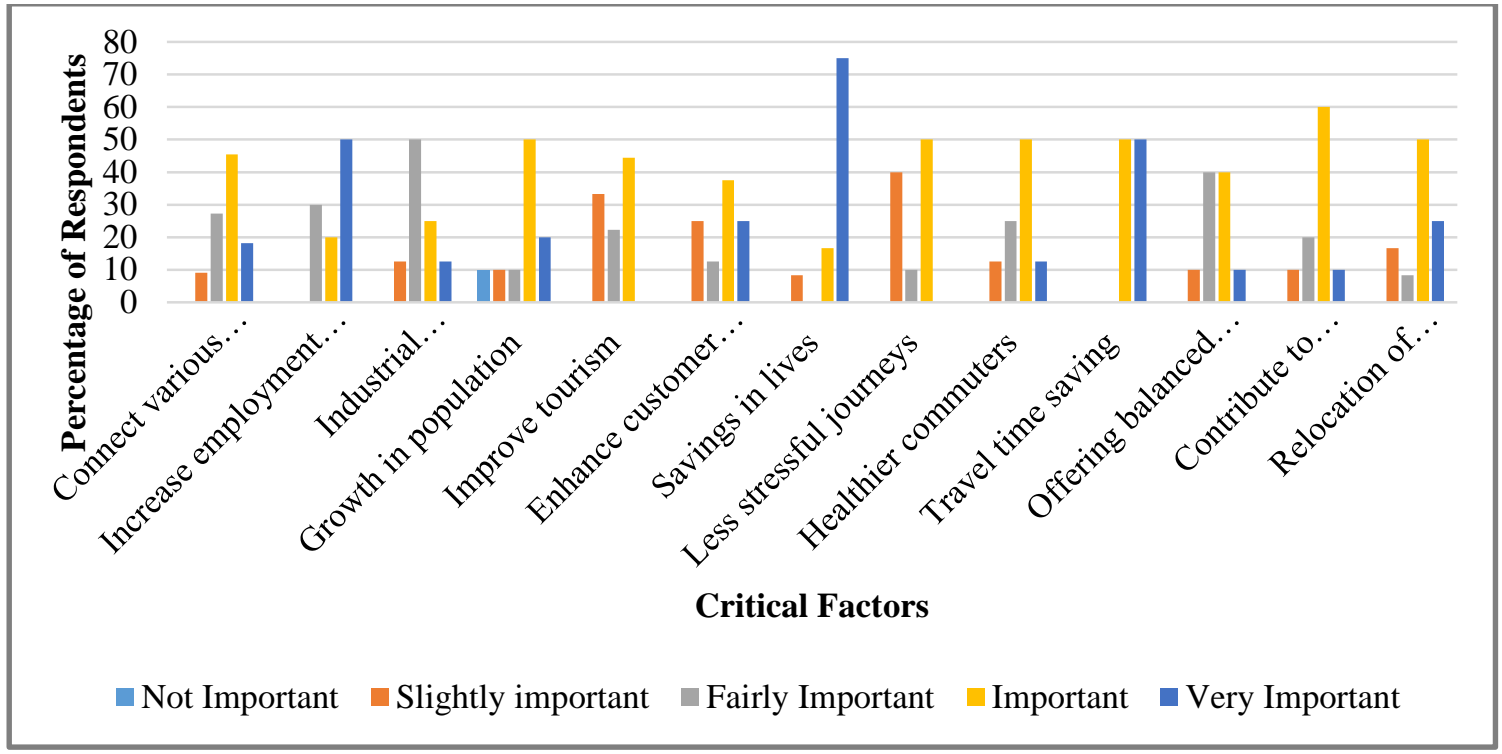

Figure 3-3: Assigned importance level of Critical Factors under "Social impacts"

Among nine critical factors under "Environmental impacts" Decision Indicator, following factors got higher importance level respectively: "Improvement in air quality", "Protecting landscape, heritage, history", "Impact of hazardous material", "Reduce greenhouse gases", "Ecological changes", and "Decrease smog and acid rains". The coming factors received less importance level respectively: "Reduce noise pollution", "Minimizing the use of land and resource", and "Use non-carbon fuel based power sources".

Table 3-5: Importance level of critical factors under "Environmental impacts"

\begin{tabular}{|c|c|c|c|}
\hline \multirow{2}{*}{ Critical Factors of "Environmental impacts" } & \multicolumn{2}{|c|}{ Is currently used in evaluation? } & \multirow{2}{*}{$\begin{array}{l}\text { Importance Level } \\
\text { (Mean value) }\end{array}$} \\
\hline & Yes & No & \\
\hline Improvement in air quality & 9 & 2 & 4.00 \\
\hline Protecting landscape, heritage, history & 8 & 3 & 3.75 \\
\hline Impact of hazardous material & 7 & 3 & 3.67 \\
\hline Reduce greenhouse gases & 7 & 4 & 3.64 \\
\hline
\end{tabular}




\begin{tabular}{l|l|l|l|} 
Ecological changes & 7 & 3 & 3.55 \\
Decrease smog and acid rains & 4 & 5 & 3.50 \\
Reduce noise pollution & 5 & 5 & 3.45 \\
Minimizing the use of land and resource & 6 & 5 & 3.45 \\
Use non-carbon fuel based power sources & 5 & 5 & 2.82
\end{tabular}

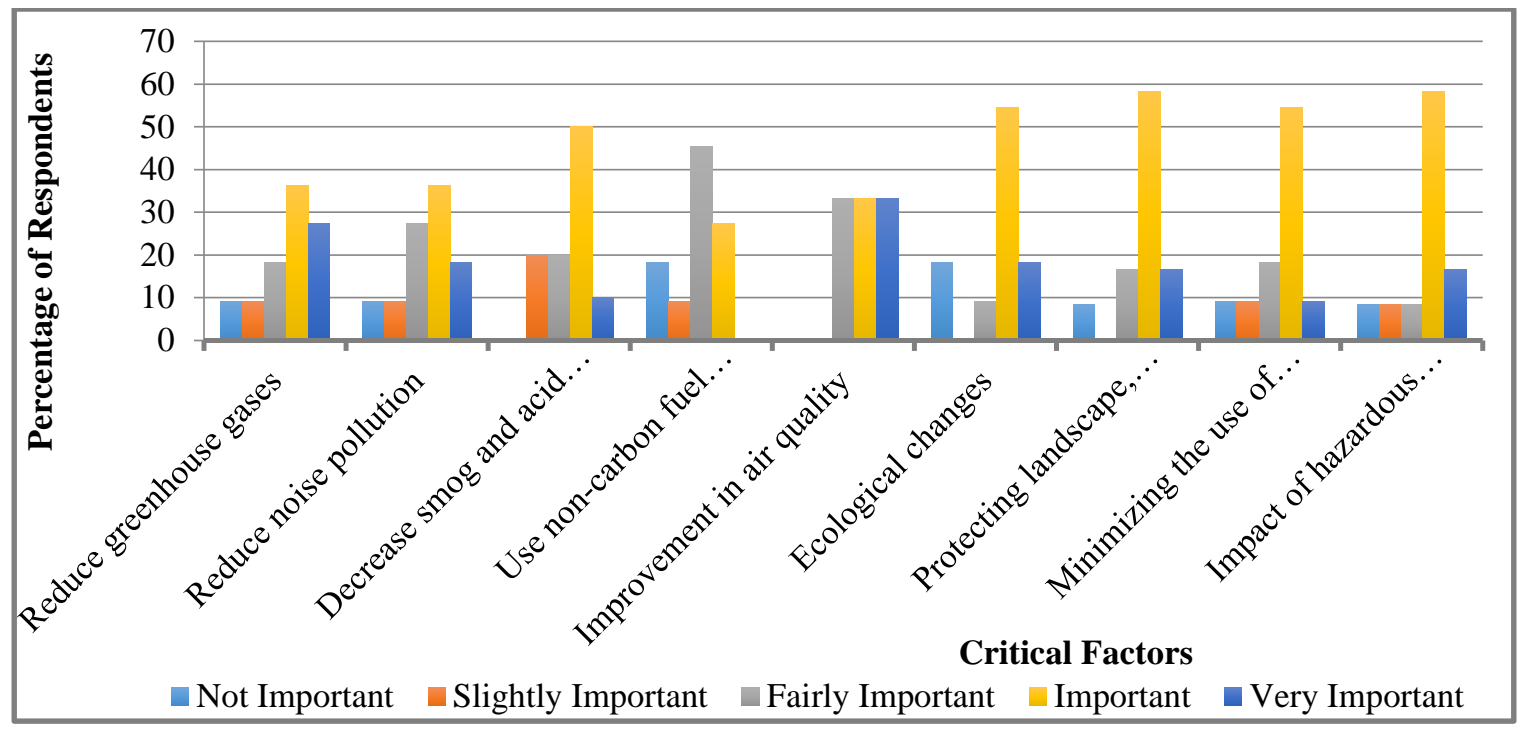

Figure 3-4: Assigned importance level of Critical Factors under "Environmental impacts"

"Construction cost" was determined as the most important factor for "Project costs" Decision Indicators following with "Land acquisition cost", "Annual maintenance cost". "Operation cost", "Planning cost" and "Cost of design" were indicated as factors with intermediate importance respectively. "Legal cost" got lowest importance level.

Table 3-6: Importance level of critical factors under "Project cost"

\begin{tabular}{|l|l|l|l|}
\hline Critical Factors of "Project cost" & \multicolumn{2}{|l|}{ Is currently used in evaluation? } & $\begin{array}{l}\text { Importance Level } \\
\text { (Mean value) }\end{array}$ \\
\hline Construction cost & Yes & No & 4.58 \\
Land acquisition cost & 11 & 0 & 3.83 \\
Annual maintenance cost & 9 & 2 & 3.70 \\
Operation cost & 7 & 4 & 3.55 \\
Planning cost & 7 & 4 & 3.42 \\
Cost of design & 7 & 3 & 3.42 \\
Legal cost & 8 & 2 & 2.80 \\
\hline
\end{tabular}




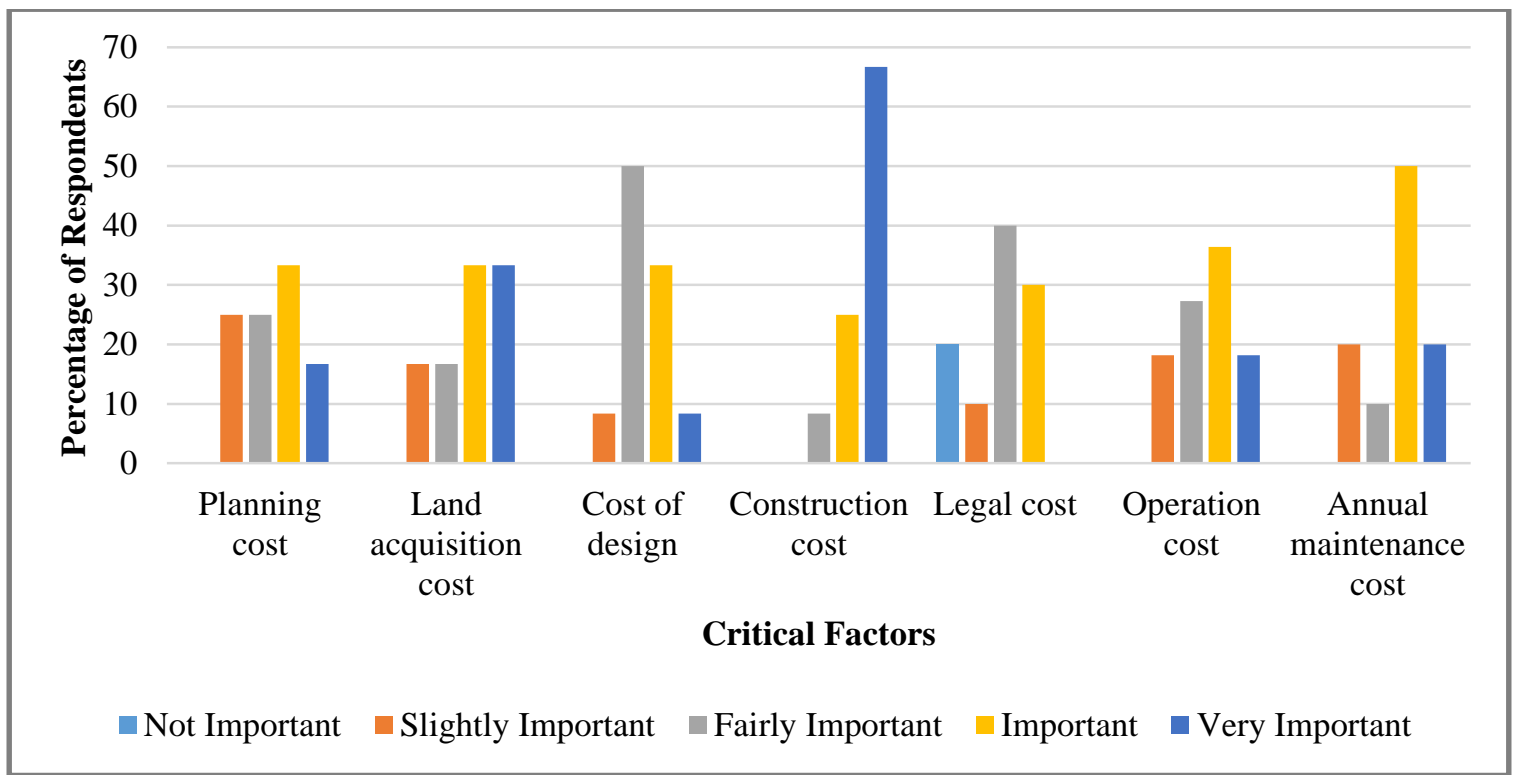

Figure 3-5: Assigned importance level of Critical Factors under "Project cost"

For "Transportation benefits" indicator, the two factors, "Reduce road accidents" and "Reduce road fatalities", were identified as the most important factors. After those "Reduce levels of traffic", "Business travel time saving", and "Reduce road operation costs" were indicated as important factor. "Cheap international travel" got the lowest importance level among all.

Table 3-7: Importance level of critical factors under "Transportation benefits"

\begin{tabular}{|l|l|l|l|}
\hline Critical Factors of "transportation benefits" & \multicolumn{2}{|l|}{ Is currently used in evaluation? } & $\begin{array}{l}\text { Importance Level } \\
\text { (Mean value) }\end{array}$ \\
\hline Reduce road accidents & Yes & No & 4.67 \\
Reduce road fatalities & 11 & 0 & 4.67 \\
Reduce levels of traffic & 11 & 0 & 4.08 \\
Business travel time saving & 11 & 0 & 4.00 \\
Reduce road operation costs & 9 & 2 & 3.58 \\
Cheap international travel & 8 & 2 & 1.67
\end{tabular}




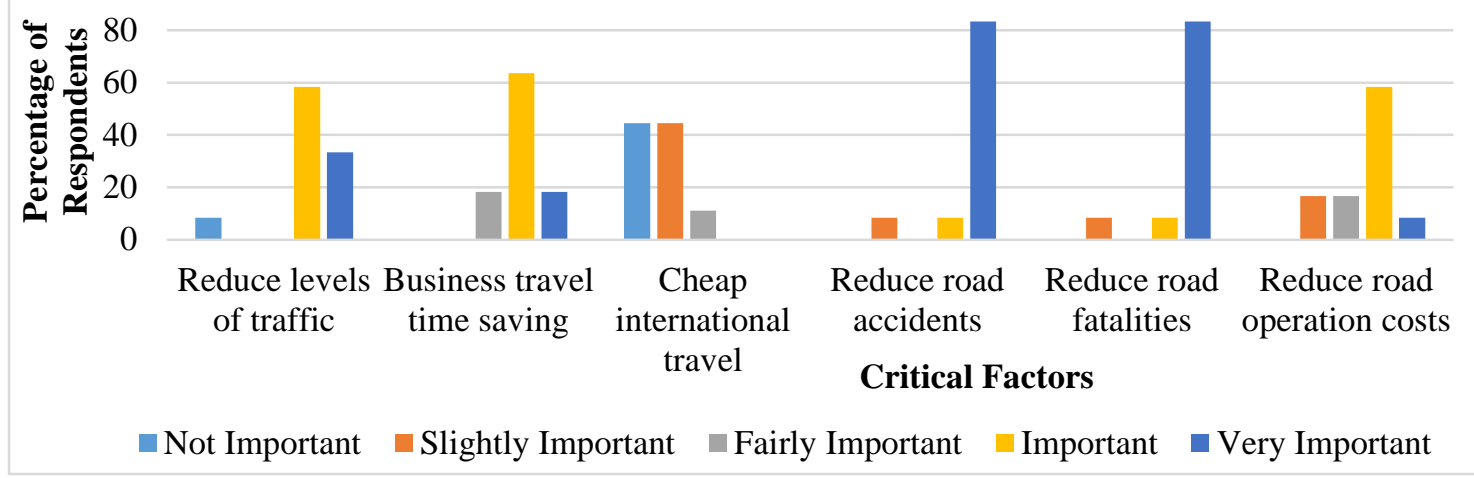

\section{Figure 3-6: Assigned importance level of Critical Factors under "Transportation benefits"}

The questionnaire responses denoted for "Technical issues" Decision Indicator, five factors out of seven are played the most important role in decision making, which are as follows respectively: "Safety during construction phase", "Project site", "Resistance to natural hazards" "Construction time", and "Availability of material and equipment". "Complexity of implementation method" and "Material Usage" were the next factors. Although most of these factors are already applied in selecting the projects, the factors related to material availability and usage are not considered. This can affect the final price of project indirectly.

Table 3-8: Importance level of critical factors under "Technical issues"

\begin{tabular}{|l|l|l|l|}
\hline Critical Factors of "Technical issues" & \multicolumn{2}{l|}{ Is currently used in evaluation? } & $\begin{array}{l}\text { Importance Level } \\
\text { (Mean value) }\end{array}$ \\
\hline Safety during construction phase & Yes & No & 4.00 \\
Project site & 7 & 3 & 3.45 \\
Resistance to natural hazards & 6 & 4 & 3.40 \\
Construction time & 6 & 3 & 3.20 \\
Availability of material and equipment & 6 & 4 & 3.20 \\
Complexity of implementation method & 5 & 5 & 3.10 \\
Material Usage & 6 & 4 & 3.00
\end{tabular}




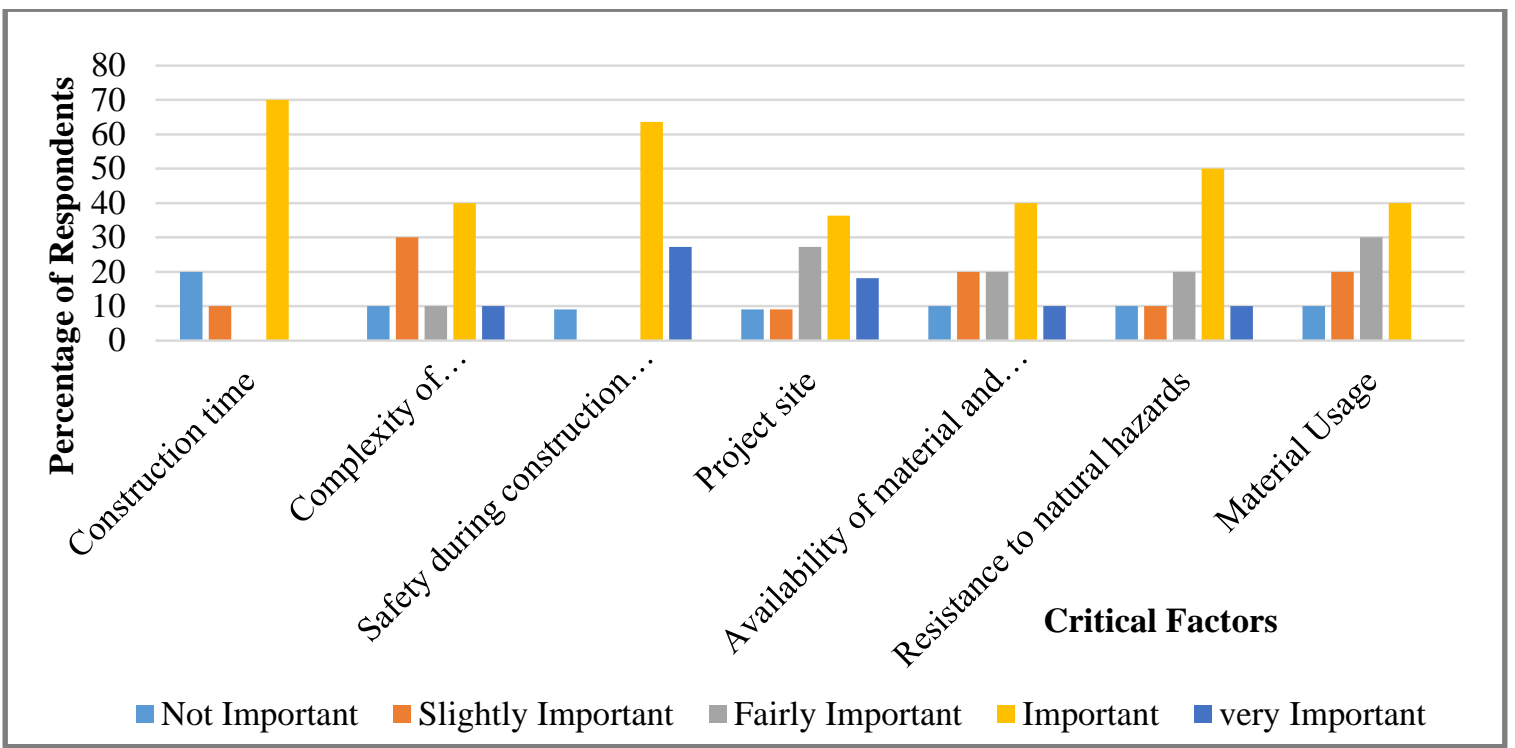

Figure 3-7: Assigned importance level of Critical Factors under "Technical issues"

Under "Financial issues" indicator, "Availability of fund" is certainly the most important factor. After that "Public-Private Partnership consideration" was diagnosed as important factor. "Legal issues" and "Interest of financial party to the alternative" got the next levels of importance respectively.

Table 3-9: Importance level of critical factors under "Financial issues"

\begin{tabular}{|c|c|c|c|}
\hline \multirow{2}{*}{ Critical Factors of "Financial issues" } & \multicolumn{2}{|c|}{ Is currently used in evaluation? } & \multirow{2}{*}{$\begin{array}{l}\text { Importance Level } \\
\text { (Mean value) }\end{array}$} \\
\hline & Yes & No & \\
\hline Availability of fund & 8 & 2 & 4.82 \\
\hline Public-Private Partnership consideration & 7 & 2 & 3.89 \\
\hline Legal issues & 5 & 4 & 3.63 \\
\hline Interest of financial party to the alternative & 2 & 6 & 3.29 \\
\hline
\end{tabular}




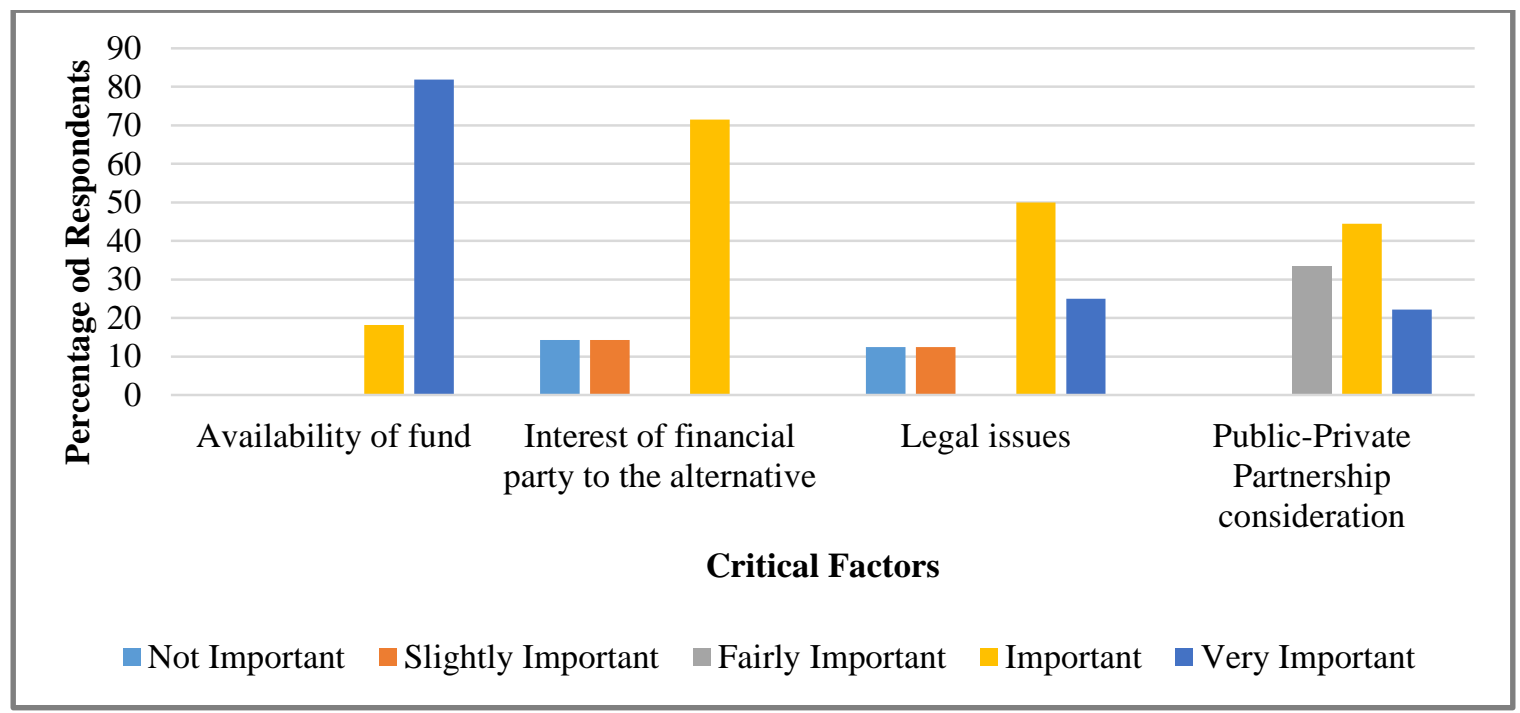

\section{Figure 3-8: Assigned importance level of Critical Factors under "Financial issues"}

The last Decision Indicator was "Political issues". Most of the respondents mentioned that this indicator is not considered in alternative selection process, however both of the factors for this category recognized as important factors: "Political discontinuity", and "Disagreement between political parties about the project". Respondents suggested other factors to be added to the list, such as: Neighborhood opposition, stakeholder (e.g., environmental) opposition, disagreement among stakeholders about project need and/or impacts; Changes in the state constitution to allow for $3 \mathrm{P}$ and to capture value added benefits. It was also mentioned that model depends a great deal on local political support. Doesn't directly measure political conflicts 


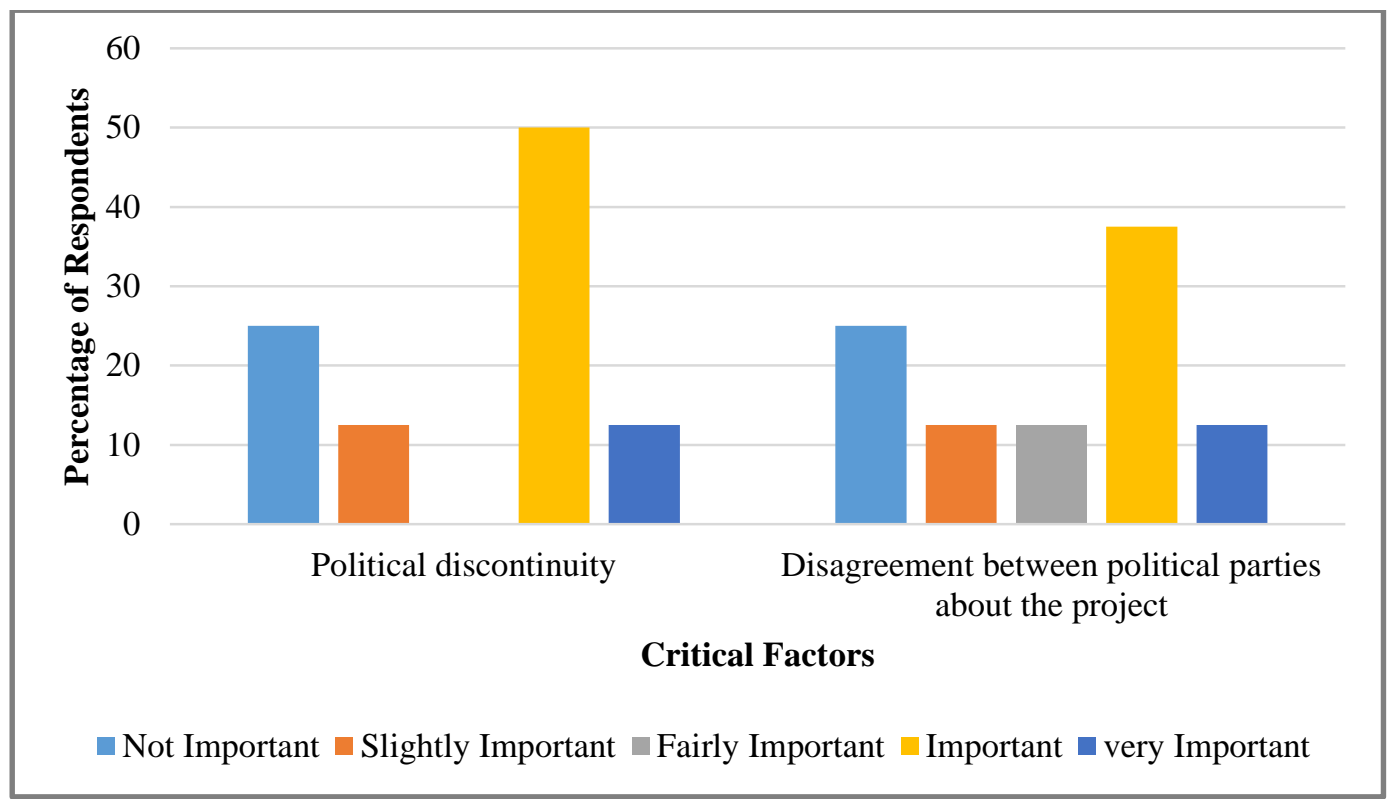

Figure 3-9: Assigned importance level of Critical Factors under "Political
issues"

Table 3-10: Importance level of critical factors under "Political issues"

\begin{tabular}{|l|l|l|l|}
\hline Critical Factors of "Political issues" & \multicolumn{2}{|l|}{ Is currently used in evaluation? } & $\begin{array}{l}\text { Importance Level } \\
\text { (Mean value) }\end{array}$ \\
\hline $\begin{array}{l}\text { Political discontinuity } \\
\begin{array}{l}\text { Disagreement between political parties about } \\
\text { the project }\end{array}\end{array}$ & 2 & No & 3.13 \\
\hline
\end{tabular}

Finally one of the respondents denoted that while the DOT may not rigorously or explicitly use some of these factors in alternative evaluation, many of them are informally/implicitly considered.

\subsubsection{Findings of Questionnaire Survey}

The results derived from this survey leads to comply the Bayesian Belief Network. Based on these results, the factors that were identified as more important factors by respondent, among poll of initial factors collected from literature review, were selected and formed the initial network for the framework. 


\subsection{Gap Analysis}

Following shortcomings have been identified in the current decision-making approaches in the governmental agencies: (i) In most of the cases, the assessment process for decision-making is in details, which needs vast data and is almost impossible to implement in the front-end phase of the projects; (ii) some of the agencies provide prioritization models to rank the project investment among the portfolio, which is useful in agency-level investment management, but not helpful in alternative assessment for a single project; (iii) lack of explicitly and transparency is observed in some reviewed agencies' process, especially in the front-end phase of the project.

\subsection{Chapter Summary}

This chapter provides an overview of the current front-end process in the U.S. governmental transportation agencies. An extensive review of legislations and published guidelines, along with a questionnaire survey has been conducted to achieve the existing alternative assessment process. Finally, a list of most relevant decision indicators as well as the factors for each decision indicator has been compiled from literature and state-ofthe practice review as a major output of this research. This list is used Bayesian belief network of the created framework. 


\section{CHAPTER 4. CONCEPTUAL FRAMEWORK DEVELOPMENT}

\subsection{Introduction}

The underlying premise of this research is to minimize the impact of uncertain conditions in the early stage of the MTPs while considering the dynamic relationship between various stakeholders, i.e. governmental transportation agencies, facility users and private funding party. As discussed earlier, finding the best solution for a transportation problem is a complex dynamic process influenced by multiple disparate stakeholders. The effective decision-making addresses multiple organizational goals and strategies using a dynamic decision process. Uncertainty and changing information, and multiple stakeholders characterize this decision environment with conflicting interests. This chapter is illustrating the theoretical development of a decision-support framework that optimize the front-end phase in the major transportation projects. The framework specifically provides the decision-makers with an assessment tool to identify the optimum alternative solution while meets the stakeholders' preferences and incorporates the uncertainty. In the previous chapter it has been described in detail that how the decision indicators and important factors are identified. This chapter gives a big picture of the decision support framework. The application of the framework will be demonstrated by a case study in the following chapters.

After navigating through the whole elicited information about different indicators and factors in the literature and real world, a list of decision indicators and the important factors under each indicator has been developed. An indicator is a parameter (or a value derived from parameters) to help quantifying the process of decision-making and present 
the complex phenomenon in an explicit manner (OECD 2007). Any indicator can be categorized as one of these three forms: component, composite or determinant/derived; which are different in the degree of specificity and its ability to be quantified. A component indicator depends on only one parameter/factor, while composite indicator measures two or more values (combined two or more component indicators). As its name implies, determinant/derived indicator integrates a concern determined or derived from other forms of indicators (Hall 2006). In developing this framework, eight simple and sound indicators, called as "decision indicators" in this research, have been identified, which are all either composite indicators or determinant/derived indicators, called as decision indicators. The decision indicators are as follows: Economic development, Social development, Protect natural environmental, Transportation benefits, Technical feasibility, Project cost, Financial feasibility, and Political feasibility.

Having the list of decision indicators, a comprehensive list of detailed critical factors that have a potential impact on those decision indicators are extracted from literature. Then the list is refined through the questionnaire survey and a series of interviews and expert opinions. Based on the interrelationships between the factors, a generic influence pattern was established to illustrate the influence of the factors on each other and also on the decision indicators. The initial list of factors is shown in Table 4-1. However, during the refinement process some changes to this list took place. For example, "Political feasibility" and all the factors related to that are deleted from the list due to experts' opinion. They indicated though it has a very important role in decisionmaking process, the "political feasibility" couldn't be considered as a "decision indicator". They believed that in most of the cases, it is neither measurable, nor 
predictable and controllable. Therefore, it has been deleted from the list. Moreover, some

of the similar factors merge together to make the final factors mutually exclusive.

Table 4-1: Initial collected Decision Indicator and Factors

\begin{tabular}{|c|c|c|c|}
\hline Economic Development & Social Impacts & $\begin{array}{l}\text { Environmental } \\
\text { Impacts }\end{array}$ & Project Costs \\
\hline $\begin{array}{l}\text { Increase employment } \\
\text { opportunities }\end{array}$ & $\begin{array}{l}\text { Connect various industries } \\
\text { and communities }\end{array}$ & $\begin{array}{l}\text { Reduce greenhouse } \\
\text { gases }\end{array}$ & Planning cost \\
\hline $\begin{array}{l}\text { Increase incomes of } \\
\text { business/property } \\
\text { owners }\end{array}$ & $\begin{array}{l}\text { Increase employment } \\
\text { opportunities }\end{array}$ & Reduce noise pollution & $\begin{array}{l}\text { Land acquisition } \\
\text { cost }\end{array}$ \\
\hline $\begin{array}{l}\text { Improve business } \\
\text { productivity }\end{array}$ & Industrial diversification & $\begin{array}{l}\text { Decrease smog and } \\
\text { acid rains }\end{array}$ & Cost of design \\
\hline Improve tourism & Growth in population & $\begin{array}{l}\text { Use non-carbon fuel } \\
\text { based power sources }\end{array}$ & Construction cost \\
\hline $\begin{array}{l}\text { Increase inward } \\
\text { investment }\end{array}$ & Improve tourism & Ecological changes & Legal cost \\
\hline $\begin{array}{l}\text { Making a greater labor } \\
\text { force available }\end{array}$ & Enhance customer service & $\begin{array}{l}\text { Protecting landscape, } \\
\text { heritage, history }\end{array}$ & Operation cost \\
\hline $\begin{array}{l}\text { Facilitating more } \\
\text { efficient trade }\end{array}$ & Savings in lives & $\begin{array}{l}\text { Minimizing the use of } \\
\text { land and resource }\end{array}$ & $\begin{array}{l}\text { Annual } \\
\text { maintenance cost }\end{array}$ \\
\hline $\begin{array}{l}\text { Extending geographic } \\
\text { markets }\end{array}$ & Less stressful journeys & $\begin{array}{l}\text { Impact of hazardous } \\
\text { material }\end{array}$ & Opportunity costs \\
\hline $\begin{array}{l}\text { Improving convention } \\
\text { business }\end{array}$ & Healthier commuters & & \\
\hline Freight cost saving & Travel time saving & $\begin{array}{l}\text { Transportation } \\
\text { Benefits }\end{array}$ & Technical Issues \\
\hline $\begin{array}{l}\text { Business travel time } \\
\text { saving }\end{array}$ & $\begin{array}{l}\text { Offering balanced regional } \\
\text { development }\end{array}$ & $\begin{array}{l}\text { Reduce levels of } \\
\text { traffic }\end{array}$ & Construction time \\
\hline $\begin{array}{l}\text { Reliability of freight } \\
\text { trips }\end{array}$ & $\begin{array}{l}\text { Contribute to development } \\
\text { strategies }\end{array}$ & $\begin{array}{l}\text { Business travel time } \\
\text { saving }\end{array}$ & $\begin{array}{l}\text { Complexity of } \\
\text { implementation }\end{array}$ \\
\hline Intermodal connections & $\begin{array}{l}\text { Relocation of residents or } \\
\text { businesses impact }\end{array}$ & $\begin{array}{l}\text { Cheap international } \\
\text { travel }\end{array}$ & $\begin{array}{l}\text { Safety during } \\
\text { construction }\end{array}$ \\
\hline $\begin{array}{l}\text { Effects to household } \\
\text { incomes }\end{array}$ & Reliability of trips & $\begin{array}{l}\text { Reduce all travel mode } \\
\text { accidents }\end{array}$ & Project site \\
\hline Financial Issues & Political Issues & $\begin{array}{l}\text { Reduce transportation- } \\
\text { related fatalities }\end{array}$ & $\begin{array}{l}\text { Availability of } \\
\text { material }\end{array}$ \\
\hline Availability of fund & Political discontinuity & $\begin{array}{l}\text { Reduce all travel mode } \\
\text { operation costs }\end{array}$ & $\begin{array}{l}\text { Resistance to } \\
\text { natural hazards }\end{array}$ \\
\hline $\begin{array}{l}\text { Interest of financial } \\
\text { party to the alternative }\end{array}$ & $\begin{array}{l}\text { Political parties } \\
\text { disagreement about project }\end{array}$ & $\begin{array}{l}\text { Increase non-SOV } \\
\text { mode share }\end{array}$ & Material Usage \\
\hline Legal issues & Neighborhood opposition & $\begin{array}{l}\text { Increase transit } \\
\text { ridership }\end{array}$ & $\begin{array}{l}\text { Equipment } \\
\text { providing }\end{array}$ \\
\hline $\begin{array}{l}\text { Public-Private } \\
\text { Partnership } \\
\end{array}$ & $\begin{array}{l}\text { Required changes in the } \\
\text { state constitution }\end{array}$ & $\begin{array}{l}\text { Travel time savings } \\
\text { for transit riders }\end{array}$ & $\begin{array}{l}\text { Providing safe } \\
\text { transportation }\end{array}$ \\
\hline Bonding rate and ability & Local political support & $\begin{array}{l}\text { Transit on-time } \\
\text { reliability }\end{array}$ & \\
\hline $\begin{array}{l}\text { Additional revenue } \\
\text { streams (ex. Tolls) }\end{array}$ & $\begin{array}{l}\text { Stakeholders disagreement } \\
\text { about project need/impacts }\end{array}$ & $\begin{array}{l}\text { Increase transportation } \\
\text { choice }\end{array}$ & \\
\hline
\end{tabular}




\subsection{Conceptual Framework}

This section introduces the conceptual framework of the prototype decision support tool that was established over the course of the research to address the problem statement. The framework was formed with three modules: 1) Alternative identification module, 2) Alternative assessment module, and 3) Alternative selection module. Figure 4-1 schemes the tasks of each module through the framework. Following sections explain each module in details.

\subsubsection{Alternative Identification Module}

Identification of project alternatives is a naturally required step to be able to start the evaluation. In the proposed framework, this first level of analysis is structured as a combination of needs analysis and scope definition. Although it sounds very clear, it is important to process these two steps precisely. Because scope deviation is one of the most seen problems in major projects, which happens when at the early stage the need analysis did not study well enough. This module will address the identified shortcomings in the existing models regarding ambiguous need identification and insufficient project scope definition. To be comprehensive, all the suggested alternatives, regardless of their effectiveness, soundness or feasibility, should be presented in this module to assess further in the next module. The outcome of this module is a list of alternative solutions by the decision-makers for that specific need. Once the project alternatives to be evaluated are identified, the next step in the framework includes evaluation of these project alternatives with respect to the above-mentioned seven Decision Indicators. 


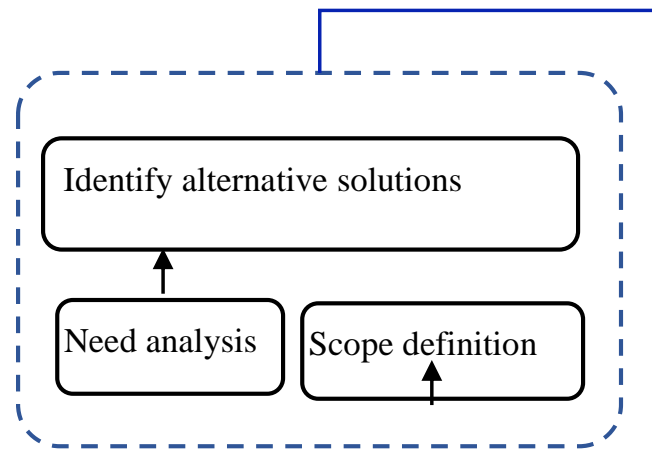

Alternative Identification Module
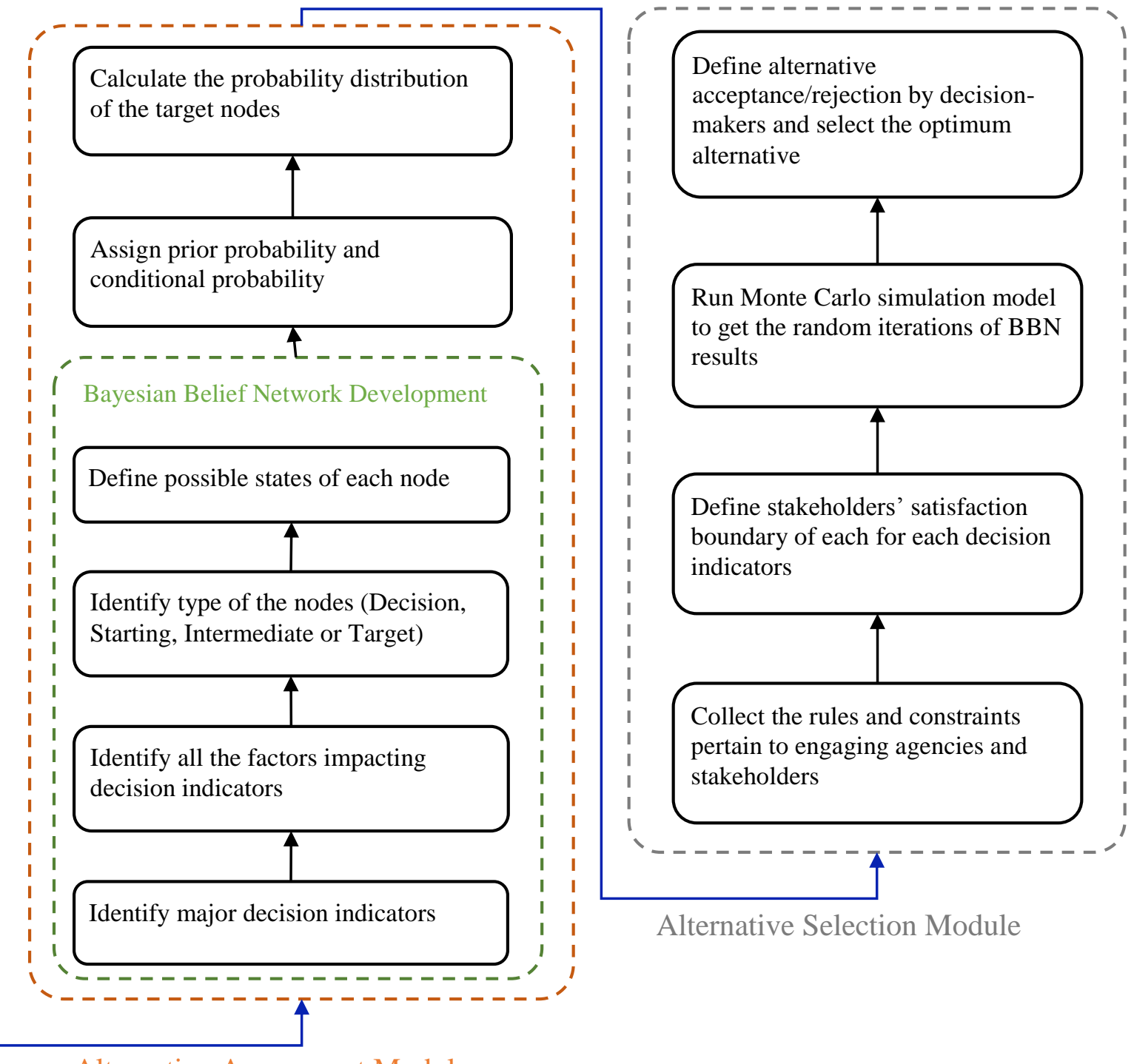

Alternative Selection Module

Figure 4-1: The proposed framework modules 


\subsubsection{Alternative Assessment Module}

The main part of the framework body is this module, which attempts to address the uncertainty of decision-making process. A Bayesian belief network, also called a causal network or belief network, is a powerful tool for knowledge representation and reasoning under conditions of uncertainty and visually presents the probabilistic relationships among a set of variables. It is frequently applied in real-world problems such as medical diagnosis, forecasting, automated vision, sensor fusion, and manufacturing control It has been extended to other applications including transportation, ecosystem and environmental management, and project risk management (Bayraktar and Hastak 2009; Lee et al. 2009; Trucco et al. 2008).

The Bayesian belief network approach has been selected as the tool for modeling MTP front-end phase process over the course of this research because it was found wellsuited for the condition this research problem. A Bayesian belief network has many advantages such as suitability for small and incomplete data sets, structural learning possibility, combination of different sources of knowledge, explicit treatment of uncertainty and support for decision analysis, and fast responses. It is therefore applied to decision support systems with uncertainty (Lee et al. 2009). For instance, that belief networks try to model the real world, i.e., not the expert. A belief network model includes an explicit representation of the relationships among the variables, factors, processes, and events in the proposed framework for the problem. Also, construction risks are not always independent or additive. The impact of two events in a construction management environment is not always the sum of their individual impacts (Nasir et al. 2003). Moreover, it is indicated in the literature that the graphical nature of belief networks 
allows variables to be added or removed from the network without impacting the rest of the network. This feature becomes possible if the modifications to the network are isolated. Additionally, rule-based expert systems allow information or evidence to be fed only at specific entry points, and the output information is generally not changing (Bayraktar and Hastak 2009).

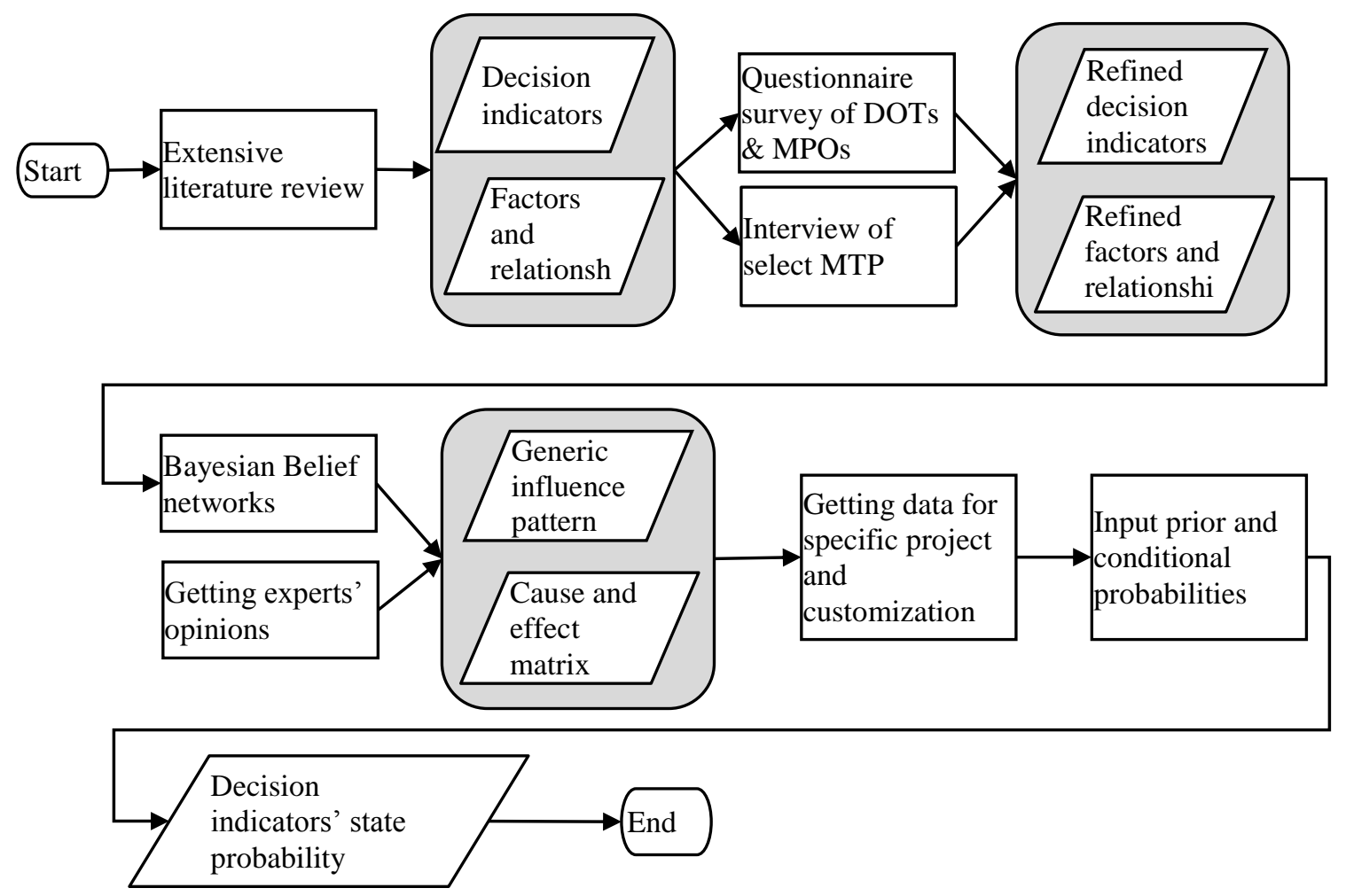

Figure 4-2: steps within Alternative Assessment Module

The Bayesian Belief Network (BBN) model is proposed as an evaluation method to address the uncertainties and interrelationship between the factors. The identified critical factors and Decision Indicators in previous research steps formed the body of $\mathrm{BBN}$. The BBN is able to simulate the impact of interrelated factors (cause-effect relation) under uncertain situation using the conditional probability theorem. The methodology and steps to create this module is summarized in Figure 4-2. 
As mentioned in chapter 2, a Bayesian network can be described in terms of a qualitative component, consisting of a directed acyclic graph (DAG), and a quantitative component, consisting of a joint probability distribution that factorizes into a set of conditional probability distributions governed by the structure of the DAG. The first component of the network is shown in Figure 4-3 schematically.

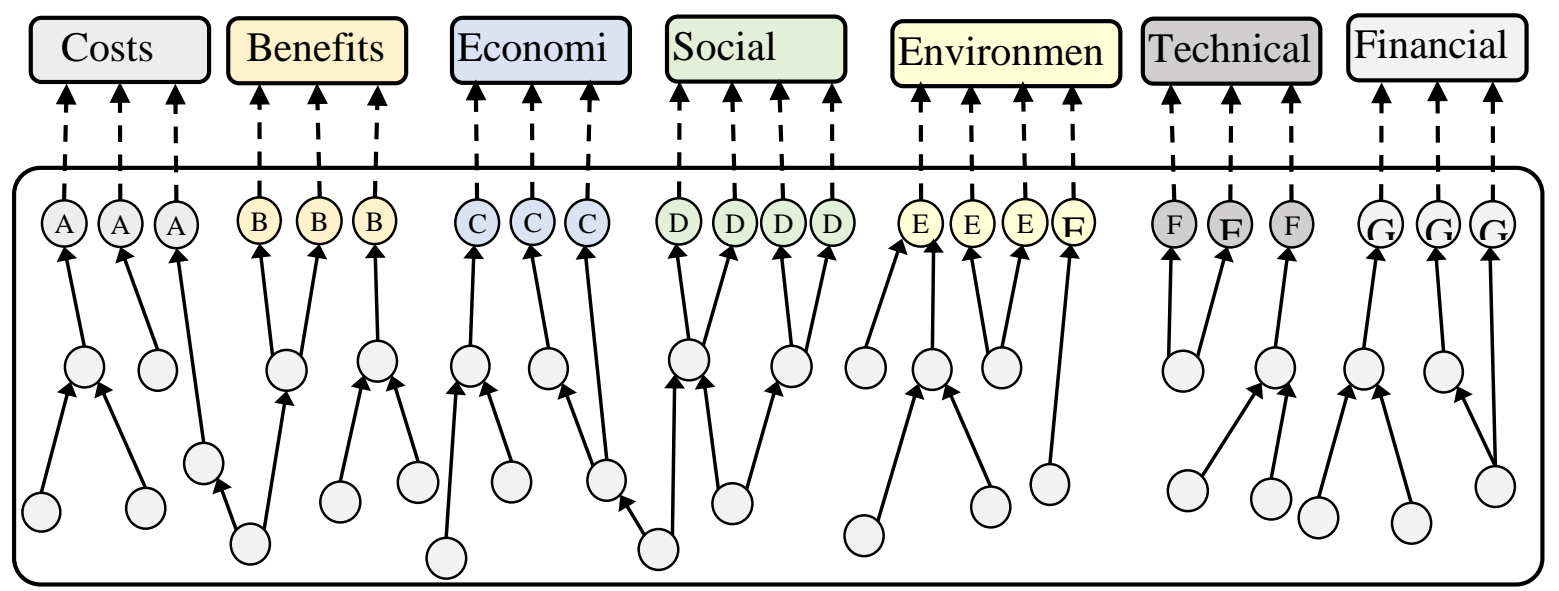

Figure 4-3: Schematic Bayesian belief network for decision indicators

The construction of a Bayesian network thus runs in two phases. First, given the MTP alternatives at hand, the relevant factors and decision indicators and the (causal) relations among them are identified (results in the generic BBN shown in Figure 4-3). This generic BBN is unique for each project based on its specification and should be customized for it. The resulting DAG specifies a set of dependence and independence assumptions that will be enforced on the joint probability distribution, which is next to be specified in terms of a set of conditional probability distributions. As explained in chapter 2 , if there is an arrow (edge) from one node to another node, then the node in the starting point of arrow is a parent of the node in the tail. If the value of a node is known, it is referred to as an evidence node. A node that has no incoming arrows is said to have no 
parents, and can be described probabilistically by a prior (or unconditional) probability distribution. A node can represent any kind of variable such as an observed measurement, a parameter, a latent variable, or a hypothesis. Relationships between variables are described probabilistically in a conditional probability table (CPT). This approach facilitates a change in the likelihood of a state of a variable to be propagated through the network. In this way, the state of the entire system can be calculated given changes in any part of it (Bayraktar and Hastak 2009; Trucco et al. 2008; Kjaerulff and Madsen 2008).

To illustrate the Bayesian belief network and interrelationships between the identified factors, a simple example of is provided here. Figure 4-4 depicted a belief network for project cost. "Project Costs" decision indicator would be influenced by "investment cost" and "operating and maintenance cost". "Investment costs" is associated with "planning cost", "cost of design", "land and property cost" and "cost of construction". "The cost of land and property" can be analyzed as "land acquisition costs" and "legal costs".

By focusing on "land and property cost" part of this example (highlighted nodes and arrows in Figure 4-4), that has four variables, we can show the network elements on it. "Legal cost" and "Land acquisition cost" are parent nodes and "land and property cost" acts as child node here. The important point that becomes obvious in the network is that once the value of "Land and property cost" is available, "Legal cost" and "Land acquisition cost" values are not necessary to predict "Project cost" values. This conditional independence is presented by the absence of a directly connecting arrow between "Legal cost" or "Land acquisition cost" and "Project cost". This feature simplifies the modeling process by facilitating the development of separate sub-models 
for each conditional relationship indicated by the presence of an arrow. These sub-models may be acquired from either (i) mathematical representation of dominant processes, (ii) statistical associations derived from historical data, or (iii) probabilistic quantities elicited from scientific experts. Any model representation or level of mechanistic detail is appropriate as long as the uncertainty associated with each relationship can be calculated using a conditional probability distribution.

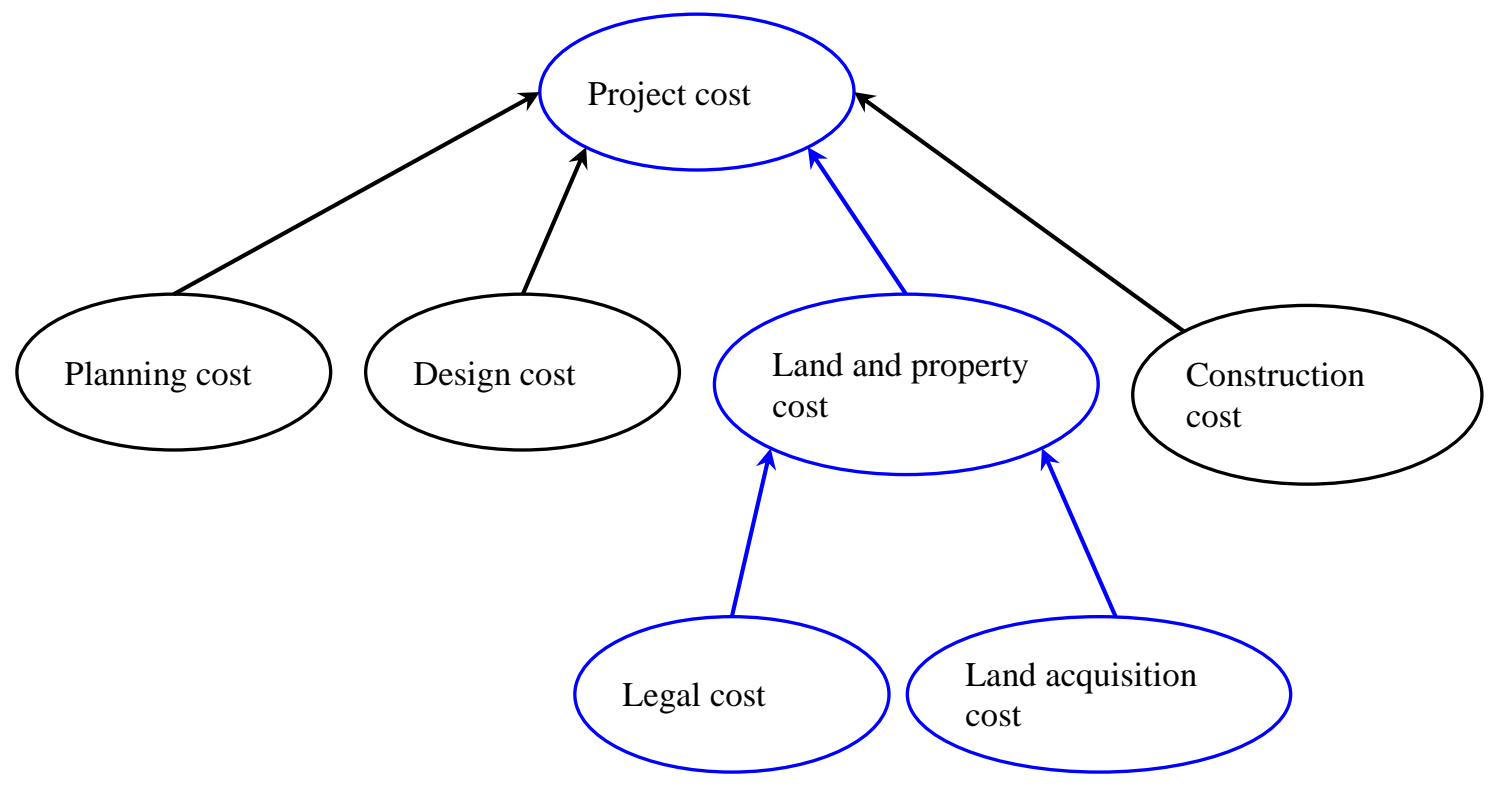

Figure 4-4: Simple project cost belief network

Once all significant system variables are linked in a single network using conditional probabilistic relationships, predictive distributions of model endpoints can be generated for any set of values for up-arrow variables. These predicted endpoint probabilities, and the relative change in probabilities between alternative scenarios (corresponding to changed values of other variables) convey the expected system response to management while accounting for predictive uncertainties (Kjaerulff and Madsen 2008). 
There are various types of software to build and calculate the probabilities of BBN available in the market. For this research, AgenaRisk is utilized to run the second module.

To provide flexibility in modeling the complexity of the problem and also to simplify the computational efforts, some assumptions have been considered. Generally, three assumptions were applied in developing the Bayesian belief network. Firstly, the nodes with at least one parent node in the influence pattern, are only conditionally dependent on their parent nodes. This kind of nodes can only be influenced by its parent(s). The second assumption indicated that starting nodes which have no parent nodes, represent prior probabilities which may be provided by the user. The other factors which are not shown in the influence pattern but may impact the project indicators and are called external factors, can only impact the starting nodes directly. Such impacts are integrated in the probabilities of the starting nodes and are further reflected in the children nodes of the starting nodes through conditional probabilities. Lastly, it was assumed that the factors provided in the influence pattern provide a mutually exclusive and collectively exhaustive list of variables required for the analysis as necessary for the use of certain probability distributions throughout the analysis.

The definition of the influence pattern as a "closed" system imposed the first and second assumptions, which is regarding the influence of the external factors only through the starting nodes. These assumptions were essentially needed to form an organized environment in order to compute the influence of the nodes on each other, and prevent getting lost in the complex nature of possible relationships between the external factors and the factors included in the influence pattern. 
A conditional probability is a probability or likelihood of a variable that is dependent on the state of another variable. Belief networks use Bayes' theorem defined as:

$$
P(B \mid A)=\frac{P(A \mid B) * P(B)}{P(A)}
$$

Bayes' theorem may also be used to analyze multiple influences as:

$$
P\left(B_{i} \mid A\right)=\frac{P\left(A \mid B_{i}\right) * P\left(B_{i}\right)}{\sum_{k=1}^{n} P\left(A \mid B_{k}\right) * P\left(B_{k}\right)}
$$

\subsubsection{Alternative Selection Module}

The last module of the framework attempts to find the best alternative using the output of the previous module, which is a set of state probabilities for 7 decision indicators. In this part a multi-criteria decision-making model is needed to find the best choice due to presence of different stakeholders even with conflict interests. The selection process in this condition is very challenging due to lack of precise data for alternatives and its inherent ambiguity, as well as difficulty in satisfying different parties in the decision-making process. There are variety methods of selecting an alternative among number of alternatives based on forcing criteria and constrains to achieve an optimization goal in the literature. However, there is clearly no single selection method that can satisfactorily and unequivocally evaluates all complex aspects of choice possibilities. The choice of assessment methods in any given choice context therefore depends on the features of the problem at hand, on the aims of the analysis, and on the underlying information base. The proposed evaluation methodology gives insight into the above-mentioned aspects that determine the choice of the appropriate evaluation method or combination of evaluation methods. By means of systematically structuring the 
evaluation process, the methodology ensures the compatibility between the assessment method(s) used and the actual problem to be tackled (Vreeker et al. 2002). In the course of this research, a selection method has been created by adopting the idea of combination of Flag model with Monte Carlo simulation.

This module is a kind of multi-criteria decision making model while incorporating uncertainty by using the input of $\mathrm{BBN}$ and calculating the probable conditions stochastically. The fundamental framework of the method is based upon two kinds of input data: a conversion matrix (structured information table) and a set of (sometimes politically determined) constraints. The conversion matrix is composed of elements that measure the satisfaction of each stakeholder for each considered alternative in relation to each relevant decision indicator. The set of constrains incorporates information concerning the relative satisfaction of the decision indicators in the evaluation. In case there is a prioritization of decision indicators in the evaluation process, a set of relevant weights should be assigned as numerical weight value.

Adopting Flag model's idea, the main purpose of the created model here is to analyze whether one or more alternatives can be classified as acceptable or not in the light of a-priori set of constraints for each stakeholder. The model does so by comparing different alternatives with a set of reference values (called Satisfaction Levels in this research). The model has been designed to assess the degree to which competing alternatives fulfill pre-defined standards or normative statements in an evaluation process. There are three important steps in applying the model:

Identifying a set of measurable indicators (same decision indicators as the previous module); 
Establishing a set of normative reference values (satisfaction levels or standards);

Evaluation of the relevant alternatives.

The input of the model is a matrix containing multi-dimensional information on a set of alternatives-relevant variables (i.e. state probabilities of decision indicators). This matrix contains the values that the indicators assume for each alternative considered. Therefore, the methodology requires the identification and definition of relevant satisfaction levels, which are suitable for further empirical treatment in the evaluation process.

The choice of satisfaction level for each agent (party) depends on the choice problem to be addressed; in general, the indicators should be in agreement with the nature of the choice issue under scrutiny and also consider the objectives to be taken into consideration.

For each decision indicator in the model, preferably a critical threshold value has to be defined. These values represent the reference system for judging alternatives. In cases that there are conflicting views on the precise level of the acceptable threshold values, the Flag method suggested a bandwidth of critical threshold values - by way of sensitivity analysis - to be used in the analysis. But in this research, a single threshold on each decision indicators for each agent has been identified. Having 7 decision indicators, a matrix of 7 by 5 cells was formed for each agent. An example of this matrix is represented as follows in Figure 4-3 that the green cells show the satisfaction of the agent and red cells are not acceptable areas for the same agent (or stakeholder). 


\begin{tabular}{|l|l|l|l|l|l|}
\hline Decision Indicator & Very Low & Low & Medium & High & Very High \\
\hline Economic Development & & & & & \\
\hline Social Development & & & & & \\
\hline Protect Environment & & & & & \\
\hline Transportation Benefits & & & & & \\
\hline Technical Feasibility & & & & & \\
\hline Project Costs & & & & & \\
\hline Financial Feasibility & & & & \\
\hline
\end{tabular}

\section{Figure 4-5: An example of stakeholders' satisfaction threshold matrix}

The results of $\mathrm{BBN}$, which were used as input to this module, are a set of probability distribution (can be presented as discrete or continuous variable) for predefined states of decision indicators. These probability distributions were used to simulate the real world decision-making conditions. For example, if the result of BBN indicated that the Alt.1 will protect natural environment very lowly by 5\%, lowly by $25 \%$, medium by $30 \%$, highly by $30 \%$ and very highly by $10 \%$. It means that in the simulation iteration, $5 \%$ of the cases will be lowly protected, in $25 \%$ will be lowly protected and so on. Using the above matrix in Figure 4-5, this probability distribution could be converted to a stakeholder satisfaction choice, i.e. each alternative can be translated as satisfied/unsatisfied foe each single stakeholder based on constrains and borderlines defined by each stakeholder.

Using a Monte Carlo simulation method, the framework is able to repeat this step for many times (as requested by the model user), and find if there is coherence in the results indicating particular alternative as the best solution. In this framework the acceptance constrains for any alternative is defined as being satisfied by all stakeholders. 
However, it will give the user a very narrow range of results. The users are able to change this acceptance condition based on their specific project under consideration.

Therefore the selection module consisted of (i) identifying relevant constrains and preferences of various stakeholders for each decision indicator; (ii) converting the output of BBN to stakeholders' satisfaction-scaled values using the conversion matrix. (iii) running a Monte Carlo simulation model to create the real world condition by having numerous haphazardly selected decision indicators" value. (iv) defining a rule for alternative acceptance/rejection by decision-makers (here the accepted alternative is defined as an alternative satisfies all the stakeholders); and finally (v) selecting the best alternative as the one that has the most number of acceptance in the Monte Carlo iteration results. These steps are depicted in Figure 4-6 below.

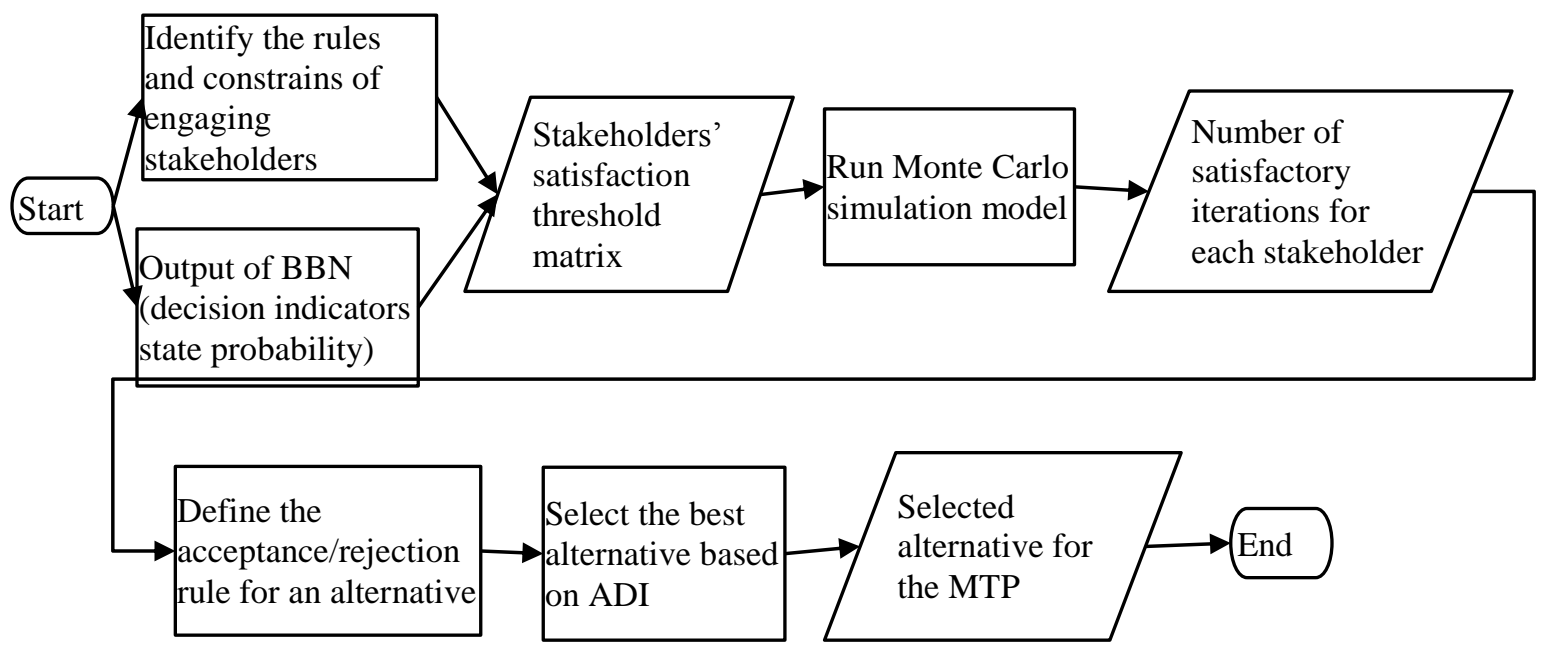

Figure 4-6: Steps within Alternative Selection Module

\subsection{Chapter Summary}

This chapter introduced the concept, framework, and assumptions of the prototype decision support tool that was established over the course of the research to facilitate the evaluation of the alternatives of MTPs in the front-end phase. Three modules were 
created to form the framework. Bayesian belief network modeling is used for module 2 and a combination of Monte Carlo simulation and Flag model is used for module 3. Concept and theory behind each module of the framework is explained by details and illustrated by an example. 


\section{CHAPTER 5. DEVELOPMENT OF FINAL FRAMEWORK FOR DECISION-MAKING IN THE FRONT-END PHASE OF MTPS}

\subsection{Introduction}

The advantages of using BBNs in uncertain condition of the front-end phase of MTPs are multi-fold. First, the capability of knowledge representation and inference under conditions of uncertainty makes BBNs an appealing tool to represent individual reasoning in decision-making. The probabilistic outcomes account for the variation inherent in parameter estimates and thus implicitly incorporate a risk component. The ability of BBNs to model causal connections between factors that shape decisions is particularly valuable for our purposes because it allows us to draw inferences about the effects of multiple decision indicators in the same time. Second, BBNs can incorporate the qualitative beliefs and attitudes of stakeholders, so-called prior knowledge, along with quantitative data. The influence diagrams are also relevant for decision-makers because they are transparent, intuitive and easy to understand. Contrary to many other simulation models, stakeholders can be more readily involved in model and scenario development, which eases their skepticism towards the modeling exercise. Compared to other graphical models, such as decision trees, BBNs have higher predictive performance and are better suited to capture the complexity of the underlying decision-making. In summary, as previously mentioned, the flexibility of BBNs in combining quantitative evidence with stakeholder information renders them an excellent extension to more rigid, rule-based expert systems that characterize an optimal production program. 
Additionally to reflect the role of different decision-makers (stakeholders) in the process, a multi criteria alternative selection method is created in the second module.

\subsection{The Bayesian Belief Network Structure}

Total of 61 factors influencing the decision-making in the front-end phase of major transportation projects were identified under the 7 decision indicators after refinement step. It has four levels of nodes connected together with arrows (edges), which depict the dependency among the nodes. The four levels are: (i) decision nodes (the nodes that are certain, and the decision about them are taken by the decision makers); (ii) starting nodes (the lowest level of the nodes that are not depended to the other nodes in the existing network and they may be affected only by external factors); (iii) intermediate nodes (the nodes in the middle level which have both parents and child in the network); and (iv) target nodes (the nodes in the top of the network that represent the decision indicators). Each of these nodes and the formation of total network are described in details through this chapter.

As described in the steps of module 2 of the framework in chapter 4, after completion of the identification of the factors, establishing the conditional dependence relationships among the variables was the next step in the development of the belief network. To expedite this step, the 61 factors were listed in a table, such that in the first column and in the top row grouped into the seven different categories, which were mentioned earlier (Table 5.1). Using this table enabled the identification of the relationships in a way similar to the one used in preparing precedence diagrams. The information obtained from the literature review were used to identify the relationships among the different critical factors in this step. 
Table 5-1: Dependency and relationship matrix of factors

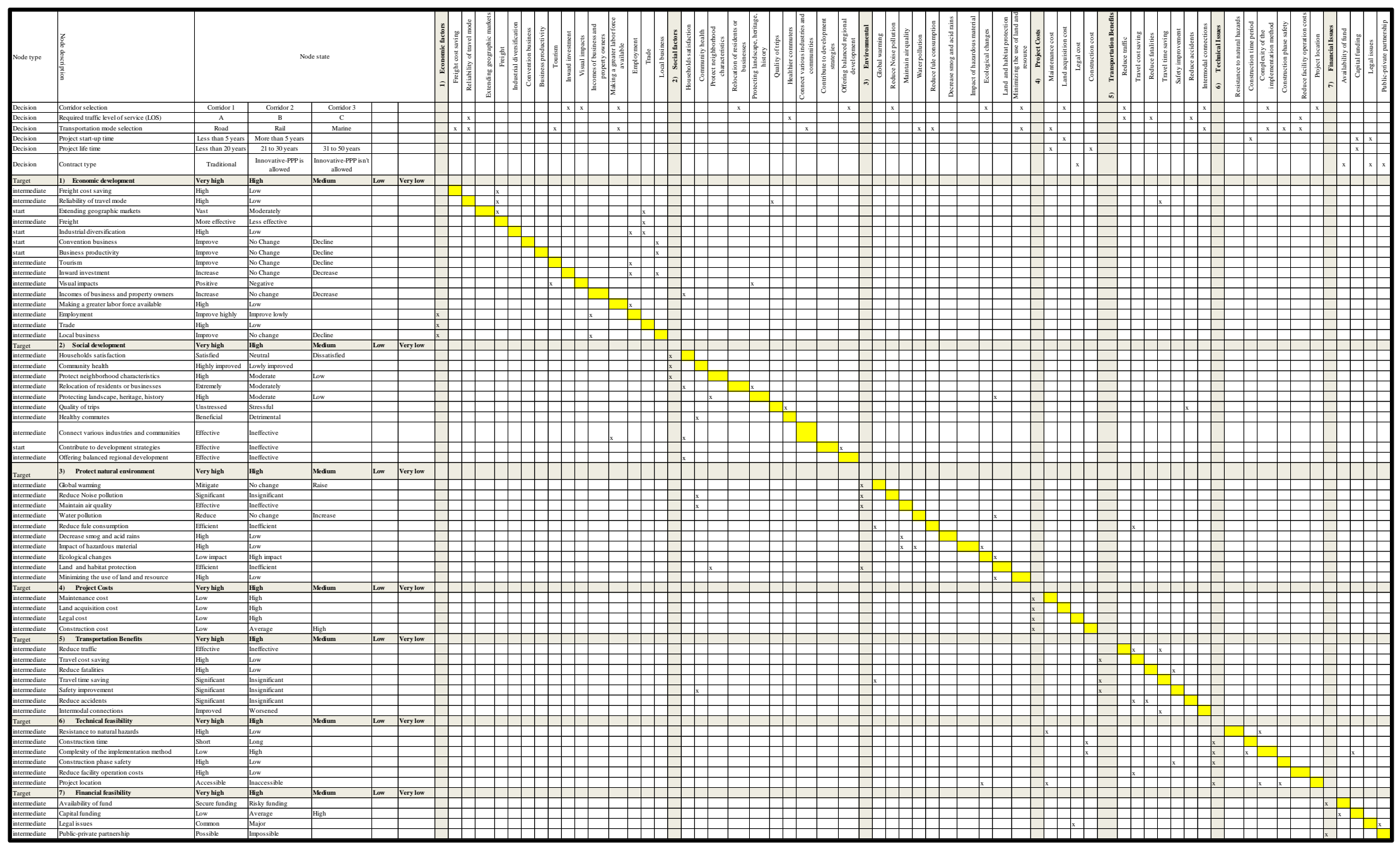


The assigned states for each node and the level of the node are also provided in Table 5-1. The relationship between the factors is also shown in this table by marking the cell that two factors are crosses.

Figure 5-1 illustrates the generic belief network established for the purposes of this research. The 61 factors identified in the previous step are presented in 61 nodes in the belief network. Also in Figure 5-1, an arrow was drawn between any two critical factors that were marked as conditionally related together in Table 5-1. These arrows represent the interrelationship between the factors visually.

Once a draft of belief network that depicted the conditional dependence relationships between the factors impacting mega transportation projects was prepared, the obtained opinion of experts from interviews were used to confirm the correctness and completeness of the proposed generic belief network. A session of model discussion was held with two experts from Florida Department of Transportation. Some factors were removed or merged from the list and some conditional dependence relationships between factors were modified based on the experts' feedback. 


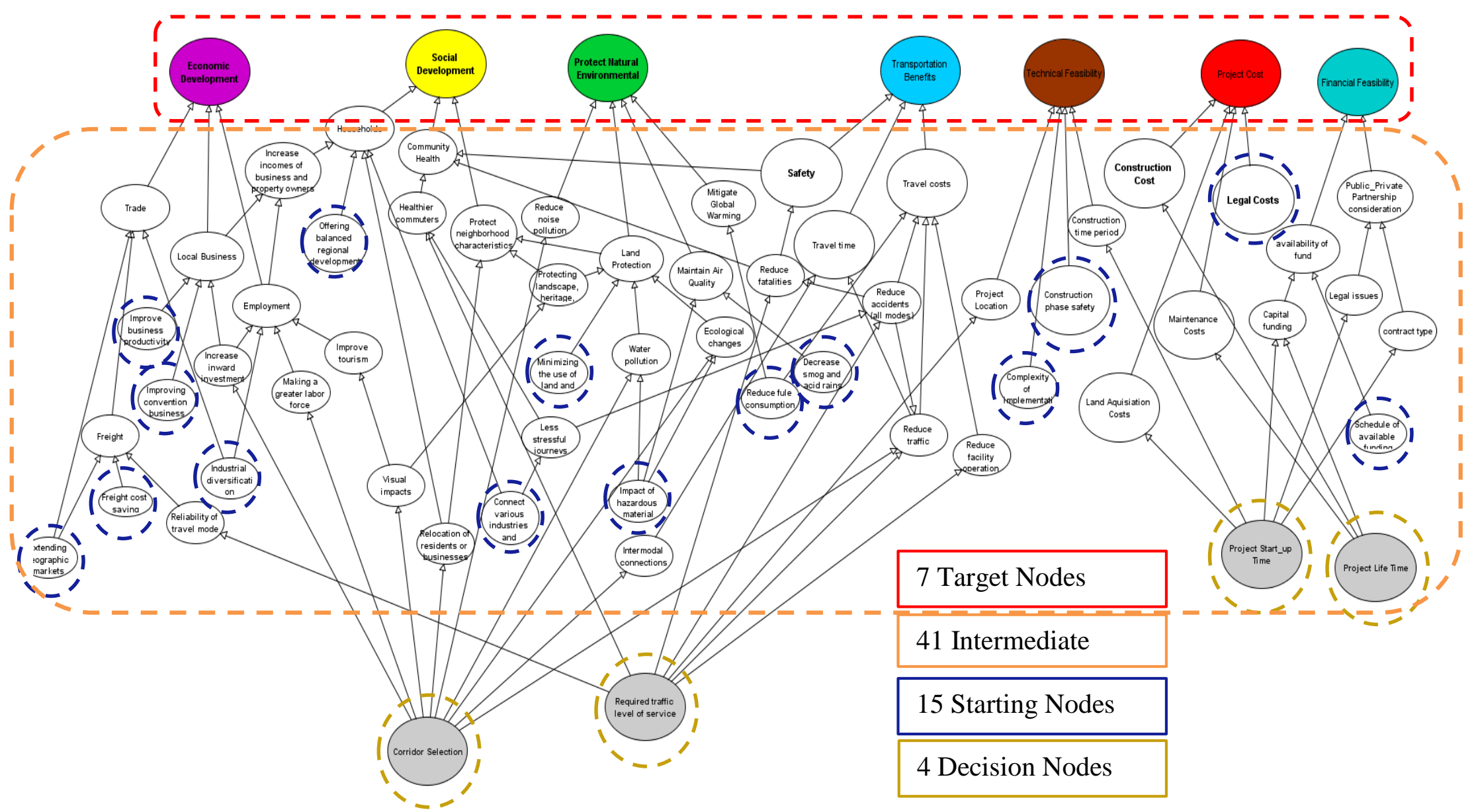

Figure 5-1: Generic cause and effect BBN with different levels of nodes 


\subsubsection{Decision Nodes}

These are a group of nodes in the lowest level of the network that the model user is able to select a state to identify the alternative under consideration. These nodes can have several states, but after input an observation and selecting a state by the model user, the prior probability of the selected state becomes $100 \%$, therefore the probabilities of the other states of that particular node should be $0 \%$. There are six decision nodes in the proposed belief network for MTP front-end phase: (i) required traffic level of service, (ii) mode choice, (iii) project start-up time, (iv) project life time, (v) contract type, and (vi) corridor choice. The decision nodes only have children and do not have parents. The state transportation agency controls these nodes states.

\subsubsection{Starting Nodes}

Starting nodes represent the nodes that have no parents and are the only the only nodes of belief network that have access to the external factors such as the inflation rate. The factors that may impact the decision indicators through the starting nodes, but are not presented in the belief network are called external factors. Although such impacts are not shown directly, they are integrated in the prior probabilities of the starting nodes and consequently are reflected in the children nodes of the starting nodes through conditional probabilities. There are four starting nodes in the belief network proposed in this research including: extending geographic markets, convention business, business productivity, and industrial diversification.

\subsubsection{Intermediate Nodes}

Intermediate nodes denote the nodes that have both successor and predecessor nodes (i.e., parents and children in the belief network). These nodes act like links 
between decision or starting nodes and target nodes and they transfer the conditional probabilities in the belief network to the top levels of the network. There are 51 intermediate nodes in the generic belief network for highway work zone projects.

\subsubsection{Target Nodes}

Target nodes point out the decision indicators of the framework. These nodes only have parent nodes in the belief network. They are the nodes on top of the network and the final output of the belief network is related to these nodes. The transition between the belief network and the next module in the model (alternative selection module) is facilitated by these nodes. The belief network proposed in this research includes seven target nodes: (i) Economic development, (ii) Social development, (iii) Protect natural environmental, (iv) Transportation benefits, (v) Technical feasibility, (vi) Project cost, and (vii) Financial feasibility. These target nodes were carefully selected to best evaluate the different aspects of the MTP decision-making process. The state of all target nodes are defined as "rank" or 5-value scale including very low, low, medium, high and very high (representing far lower than estimated impacts to very higher than expected influence, respectively).

After creating the nodes and arrows in the network, next step is input the conditional probability tables for the intermediate nodes and prior probability values for the start nodes, as well as selected stated for decision nodes. A Bayesian network can be constructed manually, (semi-)automatically from data, or through a combination of a manual and a data-driven process, where partial knowledge about structure, as well as parameters (i.e., conditional probabilities), blends with statistical information extracted from databases of cases (i.e., previous joint observations of values of the variables). 
Manual construction of a Bayesian network can be a labor-intensive task, requiring a great deal of skill and creativity as well as close communication with problem-domain experts.

\subsection{Monte Carlo Simulation and Multi-criteria Decision-making}

The ultimate objective of this framework is to provide the users with the most optimum alternative based on different stakeholders' preferences and constrains considering the uncertain condition of decision-making process. To cope with deterministic alternative selection, the created framework calculates the likelihood of seven decision indicators to have each defined state (the calculated state probability of target nodes in $\mathrm{BBN})$. To simulate the real world decision-making condition, a Monte Carlo simulation model is developed to randomly select a state for each decision indicator in each run. Then satisfactions of different stakeholders are assigned using the conversion matrix (as explained in chapter 4). This conversion matrix is defined as a multi-criteria decision-making model adopted from Flag model. In this matrix, a constraint or a boarder value is identified for each stakeholder about every single decision criteria. To illustrate the selection module application, a hypothetic example is provided here. Assuming the following Table 5-2 shows satisfaction levels inserted by one of the stakeholders:

Table 5-2: Example stakeholders' satisfaction threshold matrix

\begin{tabular}{|l|l|l|l|}
\hline \multirow{2}{*}{ Decision indicator } & \multicolumn{3}{|l|}{ Satisfaction threshold } \\
\cline { 2 - 4 } & Stakeholder 1 & Stakeholder 2 & Stakeholder 3 \\
\hline Economic development & Medium or Higher & Very high & Low or higher \\
\hline Social development & High or higher & High or very high & Medium or higher \\
\hline Protect natural environment & High or very high & High or very high & Very high \\
\hline Transportation benefits & Very high & Medium or higher & Medium or higher \\
\hline Project cost & Very low & Low and very low & Medium or lower \\
\hline Technical feasibility & High or higher & High or very high & Medium or higher \\
\hline
\end{tabular}


Once the BBN was completed and ran, the state probability of seven decision indicators were calculated as output of the model. These data was used as input for the selection module of the framework. A Visual Basic code in form of a Microsoft Excel macro was created to conduct the Monte Carlo model, and randomly select a set of states for an alternative. In this code, the state of decision indicators (target nodes) are changed to $1-5$ scale representing very low to very high respectively. The user is asked to enter the decision indicators' state probability in the given table. The ranking scale of very low to very high is replaced by a numeric scale of 1 to 5 respectively to represent the state of individual decision indicator. Then probability distribution graph of each decision indicator was drawn. In the next step the user is asked to enter the required number of iteration for Monte Carlo simulations. The program will create a set of random numbers based on number of iterations. Then a set of decision indicator states will be created for each iteration. Using the stakeholders' satisfaction threshold matrix for each stakeholder, the program will identify whether the alternative with that random decision indicators state values will be satisfactory or not. Finally a selection rule defined by the decisionmakers should be applied to recognize the accepted alternatives from the rejected alternatives. The alternative with the highest number of acceptance within the iterations will be the most desirable alternative for that particular stakeholder. To find the most satisfactory alternative for all stakeholders an "Alternative Desirability Index $(A D I)$ " is defined as follows: 


$$
A D I=\sum_{i=1}^{n} w_{i} P_{S_{i}}
$$

where $n$ is number of the stakeholders, $w_{i}$ represents the weight of each stakeholder's impact in decision-making, $P_{S i}$ is the likelihood of an alternative to be desirable or satisfactory for any individual stakeholder $\left(S_{i}\right)$. Value for the alternative desirability index will be between 0 and 1 , which higher value indicates a better alternative.

\subsection{Chapter Summary}

This chapter explains the steps for the BBN part of the final decision-making framework, i.e. the second module in the framework. First a dependency table has been developed for MTPs in the front-end phase. Then a general graphic model is presented, which was formed based on the information gathered previously. Thereafter, the categorization of the model component and the detail explanation about them was presented. Then the steps for creating the third module of the framework, which is applied to select the optimum alternative is described. The implication of the model is discussed through case studies in Chapter 6. 
CHAPTER 6. DECISION SUPPORT FRAMEWORK APPLICATION AND VALIDATION

\subsection{Introduction}

Development of the fundamental modules for decision support framework is described in details through previous chapters. The proposed framework is a general methodology with a complete set of influencing factors compiled from various references. To use the framework for a particular project, each module should be customized based on the features of the project. First, the problem to be solved by that project (need for that particular project) and relevant generated alternatives should be recognized. Then, the generic influence pattern should be customized according to collected data and project specific characteristics identified by the users. Next step is to quantify the conditional relationships in the belief network and run the BBN model to obtain the state probability of decision indicators. Moreover, different stakeholders who influence the final decision should be identified and the rules, criteria and restrictions to accept or reject an alternative to customize the satisfaction matrix. Finally the Monte Carlo simulation model should be run and using the satisfaction matrix, the best alternative can be selected. To demonstrate this procedure, and present the application of the created framework, two case studies have been done

Current chapter shows the application of the created framework using two major transportation project cases. In the first part this chapter, only the application of the assessment module of the framework is shown using the data from Port of Miami Tunnel project. Then, in the second part of chapter 6 , the entire framework is applies to the 
Detroit River International Crossing project. General information about the projects and their histories are given to emphasize suitability of these projects in checking applicability of the created framework over the course of this research.

\subsection{Case One: Port of Miami Tunnel Project}

The front-end phase in Port of Miami Tunnel took about a decade to complete although the real construction was just within 5 years. In this study the detailed process of alternative assessment and decision making along with different stakeholders engaged in this project are discussed. This study is based on a published document for the Public Affairs Team of Port of Miami Tunnel Study by Jeff V. Easley (the project manager of the previous Port of Miami Tunnel PD\&E Study) in 2003, as well as interviews with 3 persons from the project. The other required information extracted from the project website (http://www.portofmiamitunnel.com/).

For the purpose of simplification, only two alternatives (one of the tunnel alternatives and one of the bridge alternatives) have been considered and tested using BBN. Since the whole network follows the same equations and rules, only one part of the network regarding "Economic Development" is discussed in details. The objective of this example is to find out which of these two alternatives have better anticipated "Economic Development" impacts and will be a better solution from economic point of view. The BBN model calculates the probability of the predefined states of economic development of these two alternatives. 


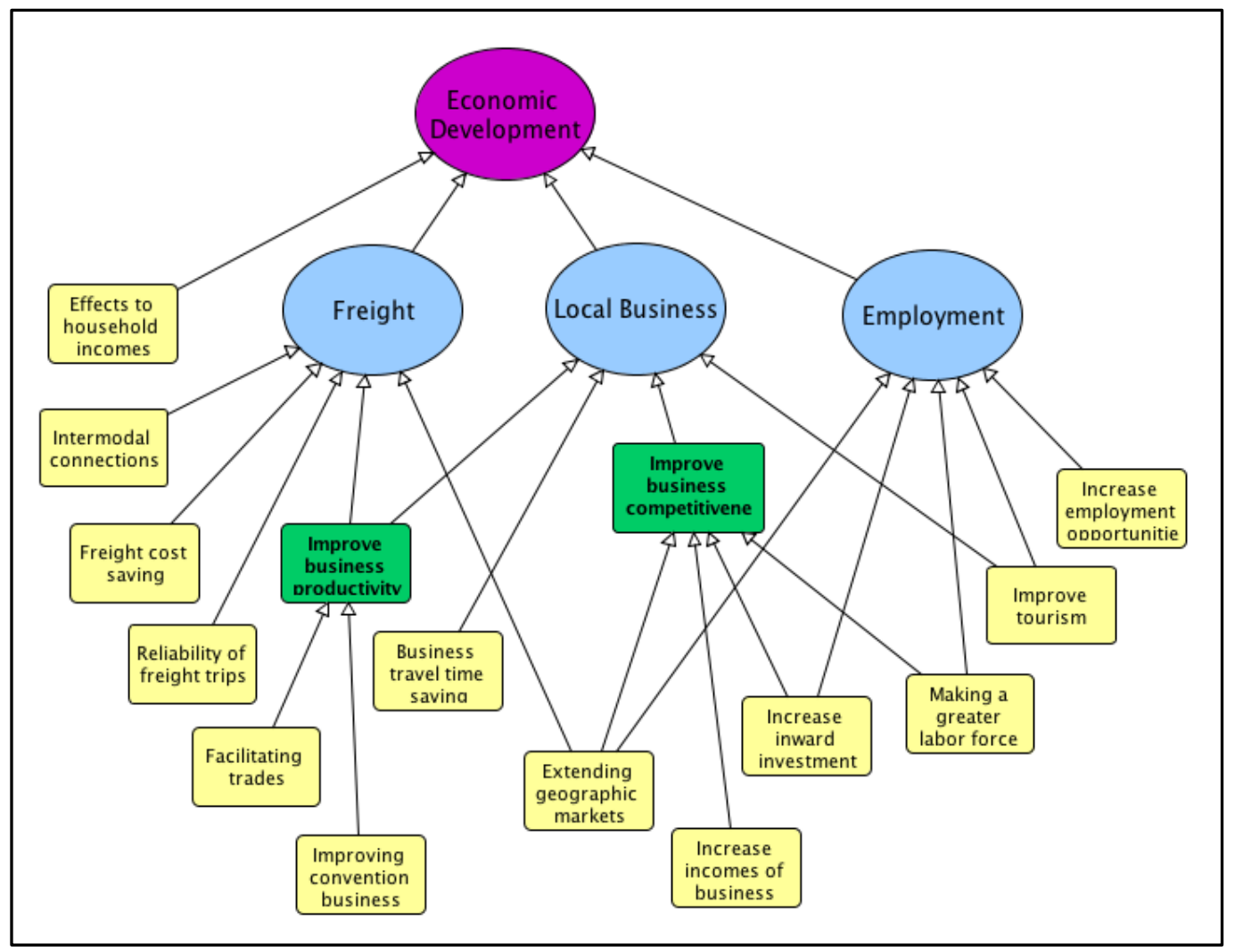

Figure 6-1: Generic relationships of economic development factors

Miami MPO identified a need for vehicular access to Port of Miami in 1980s. The project team suggested five different alternatives for evaluation including three basic alternate tunnel crossings as follows:

Alternative 1 - A tunnel running parallel to the existing Port Boulevard bascule bridge and connecting to Biscayne Boulevard.

Alternative 2 - A tunnel running parallel to the existing Port Boulevard bridge and curving to the north and running adjacent and parallel to the shoreline and connecting to I-395.

Alternative 3 - A tunnel from Port of Miami crossing diagonally under the Main Channel and connecting to MacArthur Causeway on Watson Island. 
And two potential bridge alternatives as follows:

Alternative 4 (Shore Line) - Starting at the westernmost end of Dodge Island, this corridor parallels the new Port bridge, then curves to the north and runs parallel to the shore line or the inland edge of the FEC tract and Bicentennial Park. It then joins the I- 395 corridor via another curve.

Alternative 5 (FEC Railroad) - This corridor starts at the westernmost end of Dodge Island and parallels the new Port bridge, continuing west along the FEC railroad alignment to I-95.

To simplify the calculation and illustration, only alternative 3 and alternative 4 are studied to show the proposed BBN application. Alternative 3 was a tunnel from Port of Miami crossing diagonally under the Main Channel and connecting to MacArthur Causeway on Watson Island and alternative 4 was a bridge starting at the westernmost end of Dodge Island, this corridor parallels the new Port bridge, then curves to the north and runs parallel to the shore line or the inland edge of the FEC tract and Bicentennial Park. It then joins the I- 395 corridor via another curve. Figure 6-2 depicts the location of tunnel alternative in the project site. 


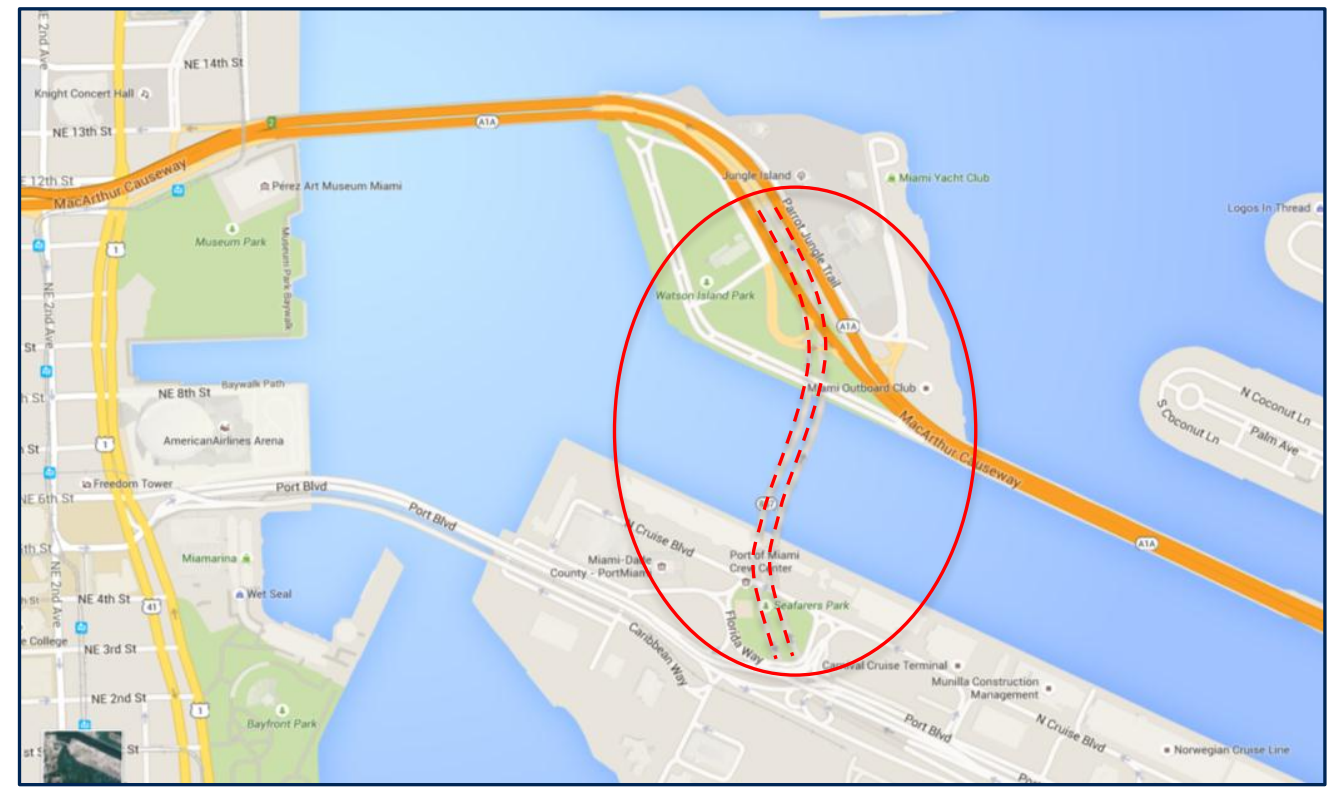

Figure 6-2: An underwater Tunnel connected to I-395 from Watson Island

Figure 6-3 also shows the schematic location of the proposed bridge as alternative 4.

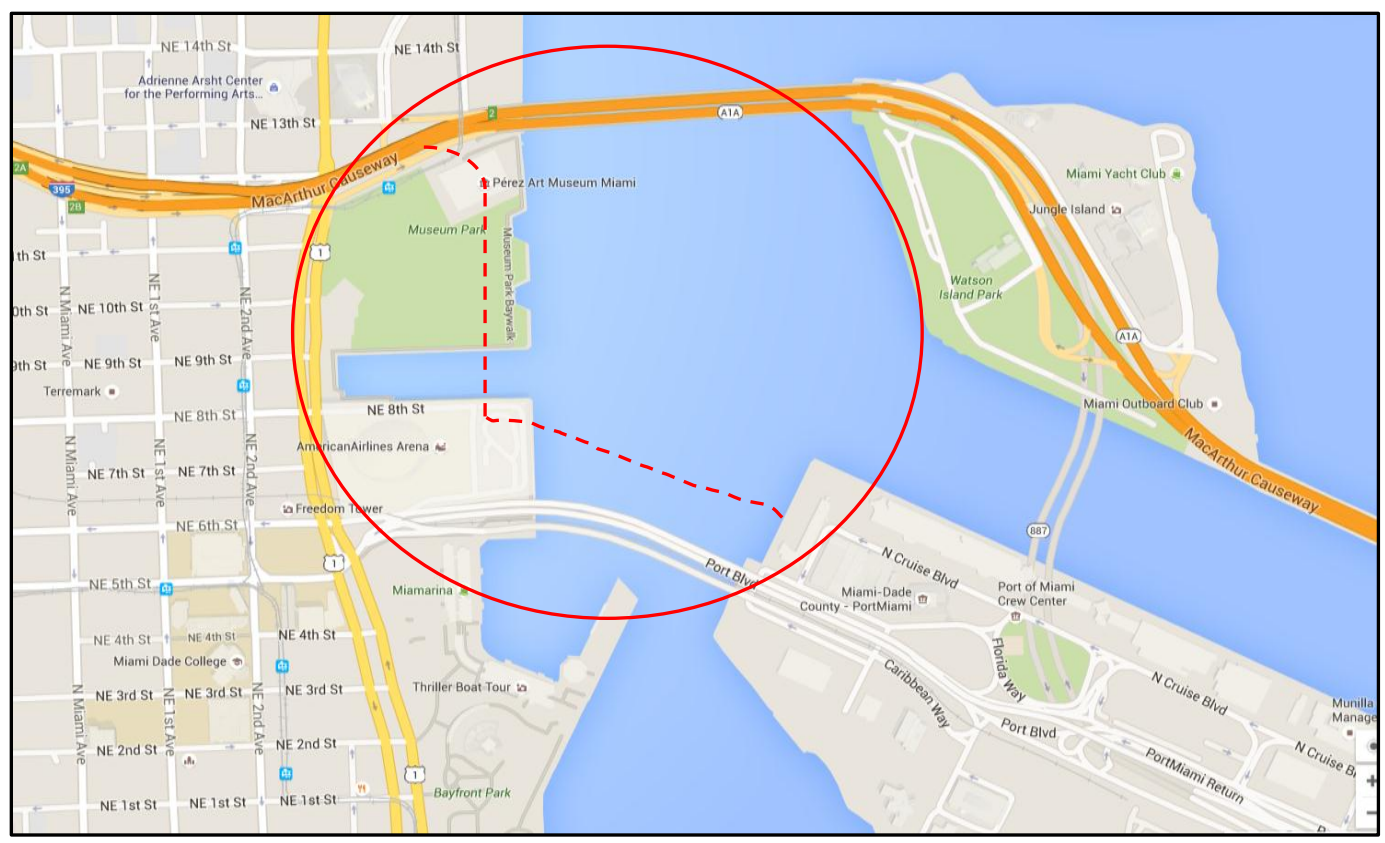

Figure 6-3: A Bridge with shoreline connection to I-395

As discussed earlier, the created network for "Economic Development" decision indicator with the critical factors under it and the relationship among the factors are 
shown in Figure 6-1. The model was run for 2 alternatives after developing the network with specific relationship between the factors based on findings from the literature review and questionnaire survey, and assigning particular states and their probability to each factor based on expert opinion through interviews.

Table 6-1 shows a sample node probability table of the dependencies in the model for one of the factors, "Improve business productivity". It includes the states for the parents' nodes and the conditional probability and dependencies between them. It shows the probability of "improve business productivity" factor to be high or low under different conditions depends on the states of parent nodes, i.e. "improving convention business" and "facilitating trades". For example the value of 0.9 in the upper left cell can be interpreted as: "There is $90 \%$ likelihood for business productivity to be lowly improved if the improving in convention business is low and facilitating trades is inefficient. Different alternatives will change only the prior probability of the parents, therefore the probability of this intermediate node will be change automatically. To simplify the numerical calculation in this application, all the factors assumed to have only two states. After identifying the dependencies and entering the prior probability of starting nodes, the probability of intermediate nodes and the target node (Decision Indicator) would be calculated by running the software (AgenaRisk is used for this study).

Table 6-1: Node probability table for "Improve business productivity"

\begin{tabular}{|l|l|l|l|l|}
\hline Factor & \multicolumn{4}{|l|}{ States } \\
\hline Improving convention business & Low & High & \\
\hline Facilitating trades & Inefficient & Efficient & Inefficient & Efficient \\
\hline Low & 0.9 & .65 & .75 & .15 \\
\hline High & 0.1 & .35 & .25 & .85 \\
\hline
\end{tabular}


Figures 6-4 and 6-5 show the achieved probabilities in the network for the two different scenarios (tunnel and bridge alternatives), respectively.

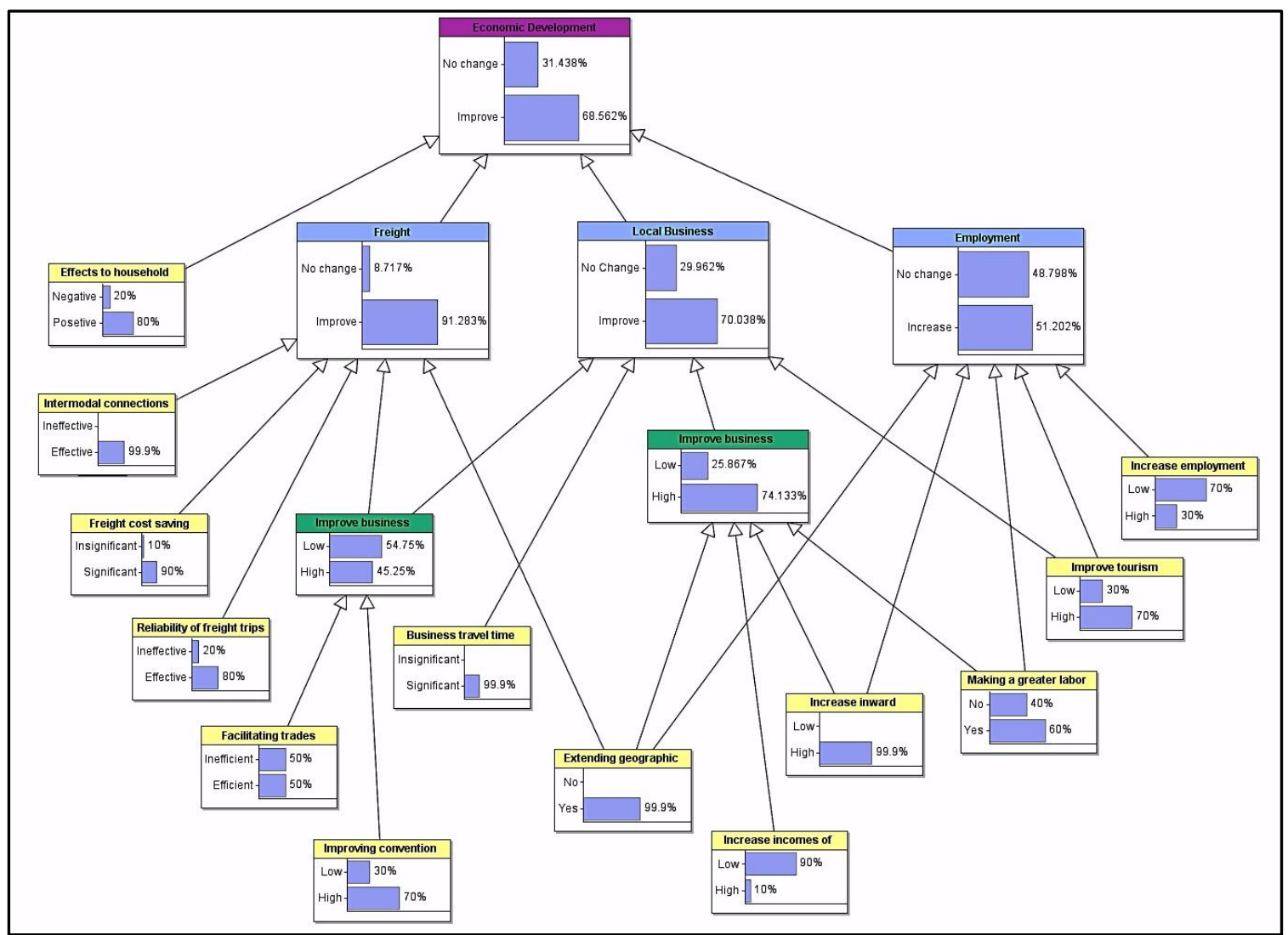

Figure 6-4: Calculated conditional probabilities for BBN nodes of Economic Development under Tunnel scenario

Based on the results of this model, shown in Figures 6-4 and 6-5, the Probability of Tunnel alternative to have improved economic development is $68.5 \%$ while for the Bridge alternative it is $61.4 \%$. This provides the decision-makers a metric to consider the uncertainties of factors involved in this Decision Indicator. The difference between the two scenarios is not significant, that was expected due to having same situation in most of the factors for both scenarios. However considering other decision indicators in the holistic model will simply differentiate the alternative scenarios. This is only a partial segment of the created $\mathrm{BBN}$ to illustrate the basic theory and calculations behind the 
belief network. The entire network includes 6 other decision indicators that will be used as input for the alternative selection module of the model.

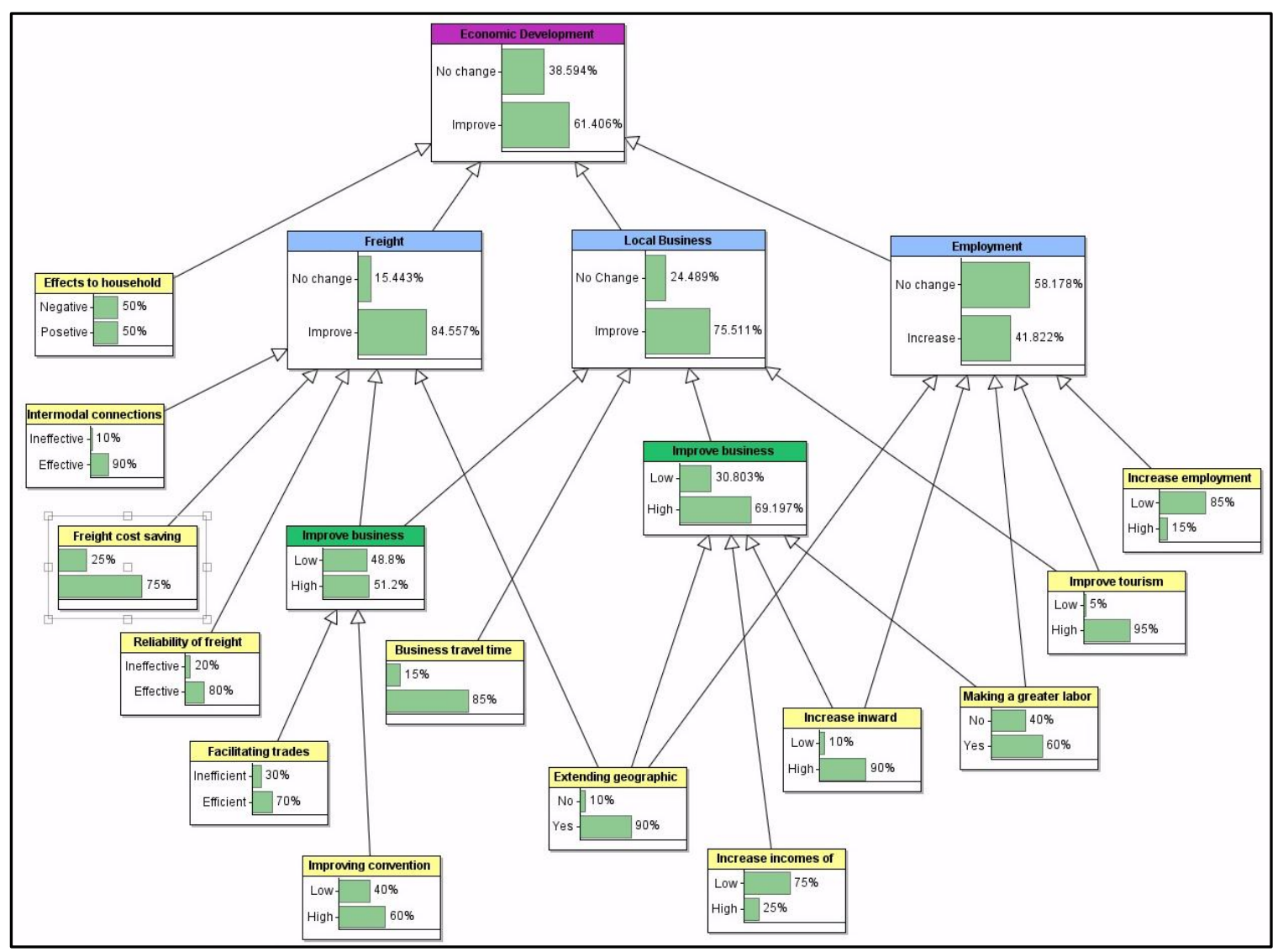

Figure 6-5: Calculated conditional probabilities for BBN nodes of Economic Development under bridge scenario

Another part of the BBN regarding "Environmental Impacts" is also presented in this chapter for the same project. The same steps as above were done to create and run the BBN for "Environmental Impacts" of two alternatives for Port of Miami Tunnel project. Figure 6-6 shows the factors and their relationship under "Environmental Network" decision indicator. 


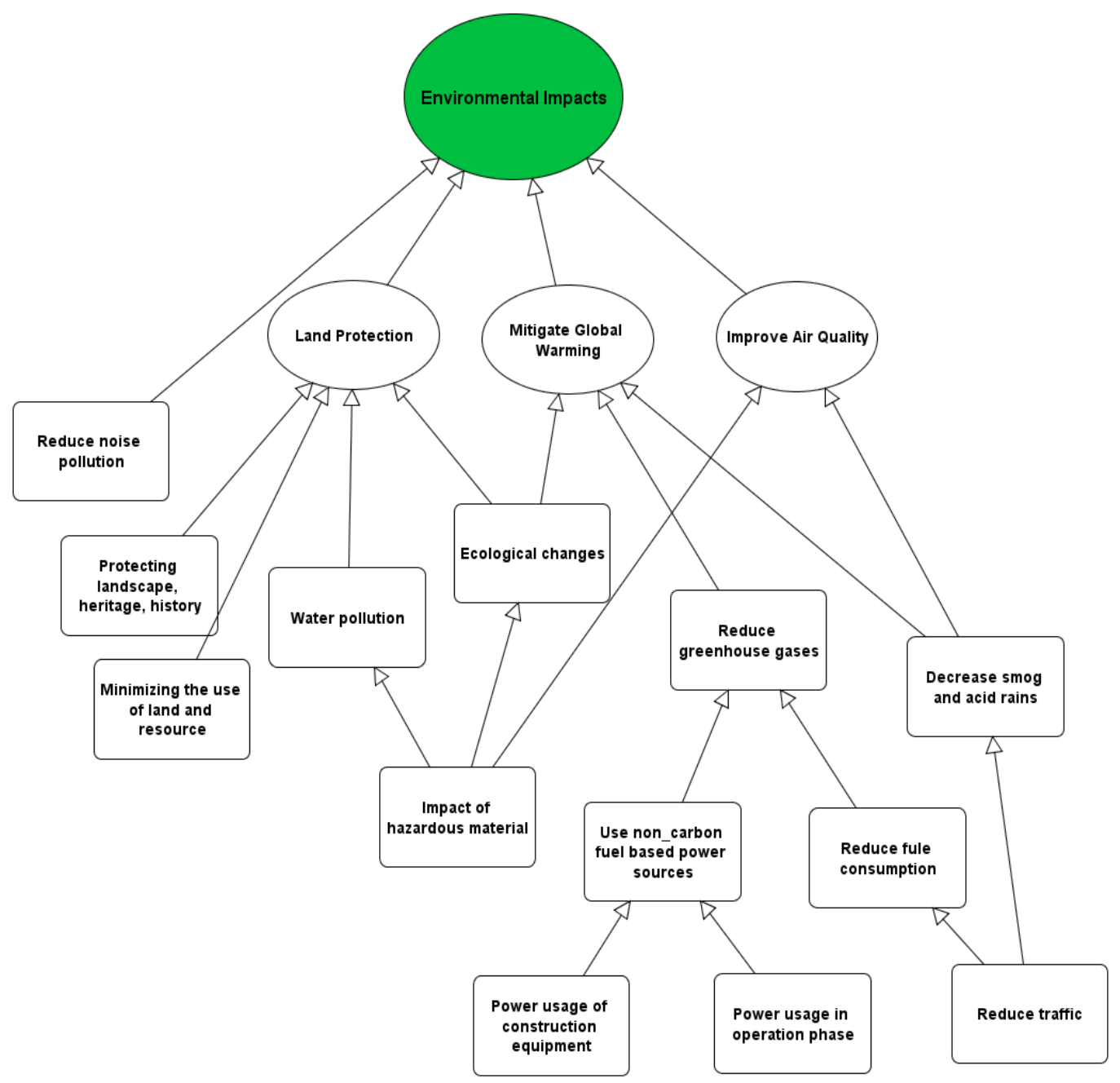

Figure 6-6: Generic BBN for "Environmental Impacts" Decision Indicator

Figures 6-7 and 6-8 show the achieved probabilities in the network for 2 different scenarios based on tunnel and bridge alternatives respectively. Based on the results of this model, as shown in the Figures 6-7 and 6-8, the Probability of Tunnel alternative to have positive environmental impacts is $56 \%$ while for Bridge alternative is $40 \%$. This provides the decision-makers a metric to consider the uncertainties of factors involved in this Decision Indicators. 


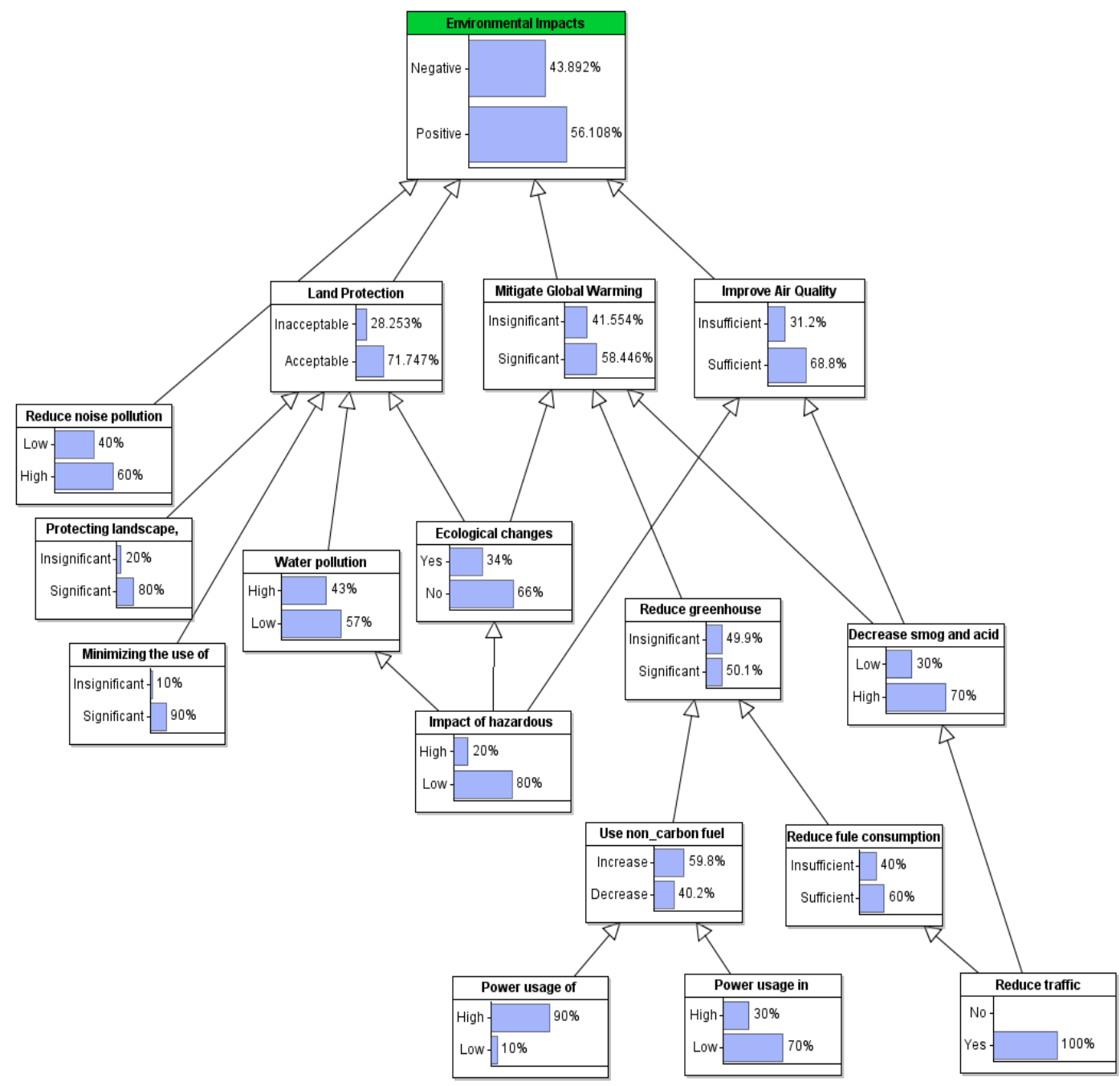

Figure 6-7: Calculated Probability for Tunnel Alternative 


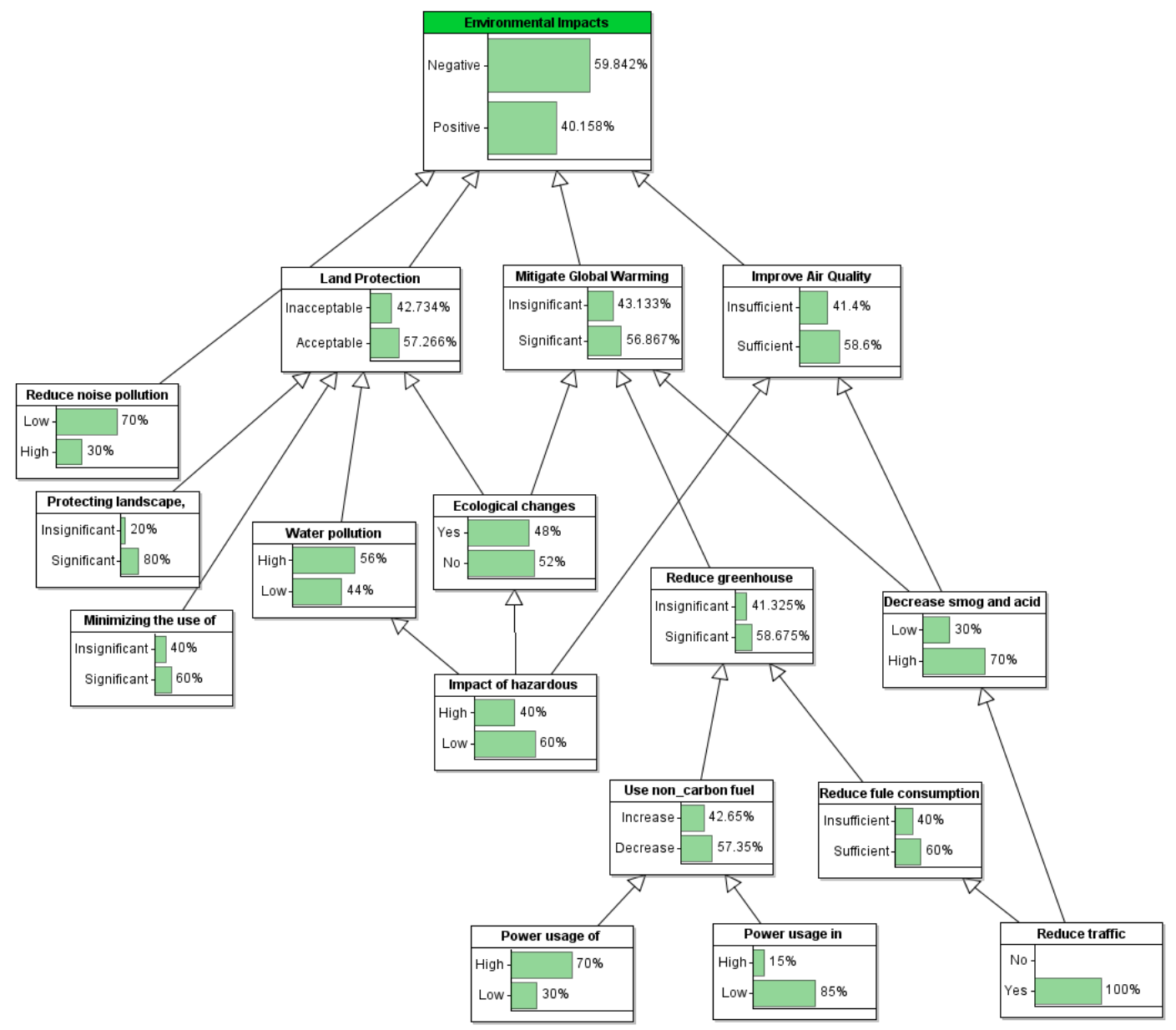

Figure 6-8: Calculated Probability for Tunnel Alternative 
Figure 6-9 is a screen shot of created node probability table in AgenaRisk software for factor "Use non-carbon fuel based power sources". It includes the states for the parents' nodes and the conditional probability of them.

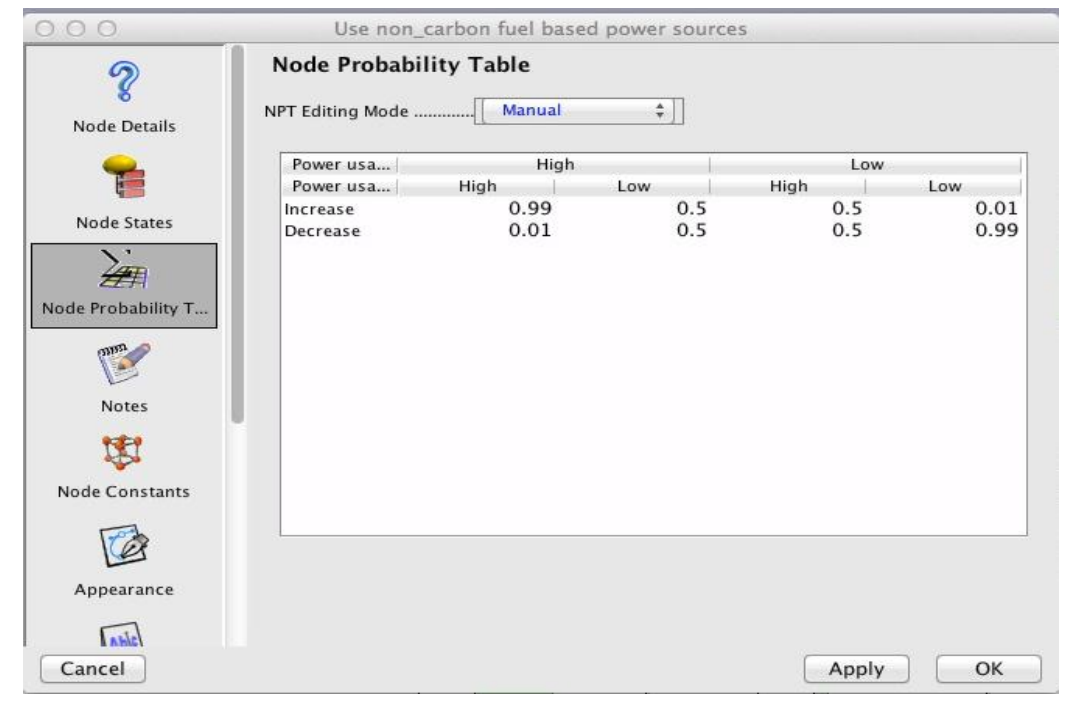

\section{Figure 6-9: A sample node probability table}

The obtained results of this application are matched to what happened in reality for the project. Based on reviewed documents of project and interview that conducted with 3 persons that were engaged in this project and worked in managerial level, in their studies the tunnel alternative got higher rank in economic development assessment.

Some highlighted points of the interviews are presented here:

- In the initial study through PD\&E (Project Development and Environmental) study, which followed NEPA process considerable environmental impacts was investigated. All of the initial alternatives were bridges since the tunnel alternative caused lots of environmental impacts (the sucking tool method with excavate and scrubbing the sea grasses in Biscayne Bay, which is very environmentally sensitive) and the technology of boring machine was not available yet. But the 
bridges alternatives faced some problems too; this port is a channel for cruise ships as well as military ships that were height up to 160 feet on that time and they were growing in height continuously. Building a bridge limited the future ships to enter the Port. Very high bridge could not be an option either, since the heavy trucks could not drive over high slopes. So it took so long to find the best alternatives. During this time, the technology advanced and boring tunnel alternative was added to the list and finally was selected as the most feasible and practical solution.

- The selected alternative contained high level of construction risks, because of the soft ground and unknown geotechnical issues. To mitigate the risk many works had been done to develop the geotechnical profile.

- In a project in this magnitude you need the support, because if you don't have the support there is a risk often, probably the industry won't even bother the bill on it if they know they're goanna have the political issue. Because that's a risk to them, they're goanna to put time and money on that to resolve any political issues. They just won't touch it; they need permit. They want to make sure they're able to get the permit. Otherwise, that's a risk to them and in order to mitigate risk, they're goanna throw money, or in other word increase the price of project. So for us as a government agency, we need to mitigate all those risks up front whether is coordinating with all the public officials and anybody that's goanna be impacted.

- Probably one of the biggest risks on the projects because of the magnitude was developing the partnership of funding, in order to get that the right amount of funding on the table to make this project viable. The extensive discussions and 
negotiations of the benefits of the project to the county and to the city of Miami certainly were made and that value a number to put on it in order to have that quantified. Then when you have to go to the political body to get the approval from the city commissionaire or the county commissionaire, that's completely out of your control. It could be a very good project, engineering wise, but again if you don't have that political champion and political support for the project where the benefits to them as the public figures and politicians, out weight any perceived these benefits. You know, if you want to put it in your risk model, how would you do that? Those are the soft but real risks to the project in making a project like this.

\subsection{Case Two: Detroit River International Crossing}

To illustrate the application of the entire created framework in a real-life project, the Detroit River International Crossing (DRIC) project is drawn on in this section. This project provides an excellent test case due to the extensive amount of detailed public information that is available on the project's early stage and planning phase. This detailed information is readily obtainable through the project website, Michigan DOT website and the project company.

\subsubsection{Project History}

The Detroit River International Crossing or the New International Trade Crossing, which is recently named as Gordie Howe International Bridge, is a planned bridge and border crossing to connect Detroit and Windsor across the Detroit River. The crossing will link Interstate 75 and Interstate 94 in Michigan with the new Rt. Hon. Herb Gray Parkway connection to Highway 401 in Ontario. 
The state of the Michigan is the principal gateway for U.S. international trade with Canada, which carries $27 \%$ of total North American land-based international trade through its three ports of entry including Detroit. These statistics shows economic importance of the study area. This project has been developed due to the growing value of surface trade between the U.S. and Canada. Studies by the Michigan DOT and Ontario government revealed that more than half of the largest bi-national surface trade crosses the Detroit River by truck. The value of the surface trading is expected to increase to nearly $\$ 240$ billion (USD) by 2030 , which necessitate the improvement of the surface link between these two countries. The project will benefit the local, regional and international economies by facilitating the trade in that root. Three connecting links are already existed in the Detroit-Winsdor area: Ambassador Bridge, Blue Water Bridge and Detroit-Winsdor Tunnel. However, due to the limited capacity of the existing links, a need for a new or improved link was identified. Moreover, after 9/11 terrorist attacks, security considerations and redundancy issues should be addressed in the major infrastructure ${ }^{1}$.

Research team from governmental agencies has begun the studies for this problem in 2000 and developed the initial feasibility study report by 2002. To address the challenges, several alternatives had been suggested and assessed by the project team. Originally 7 alternatives were suggested to meet the needs, including: 1) Do nothing; 2) Boarder processing; 3) Transportation demand processing; 4) Transit improvements; 5)

\footnotetext{
${ }^{1}$ The redundancy issues indicates to the availability of options for maintaining the movement of people and goods in case of major incidents, maintenance options or congestion at any of the current boarder crossings. Theses issues consider the network reliability along with security.
} 
Rail improvements; 6) Marine improvements; and 7) New and/or expanded roadways. A

list of alternatives with a brief explanation is provided in the Table 6-1 bellow (FHWA et

al. 2004).

Table 6-2: DRIC Alternatives Description (FHWA et al. 2004)

\begin{tabular}{|c|c|}
\hline Alternative & Description \\
\hline $\begin{array}{l}\text { The "Do-Nothing" } \\
\text { Alternative }\end{array}$ & $\begin{array}{l}\text { Defined as taking no significant action to expand infrastructure, manage } \\
\text { demand or improve operations. It contains the only transportation } \\
\text { improvements already included in the existing plans and programs for by the } \\
\text { Southeast Michigan Council of Governments (SEMCOG) and the Windsor- } \\
\text { Essex area. It will not improve the existing border processing capacity. }\end{array}$ \\
\hline $\begin{array}{l}\text { Improvements to } \\
\text { Border Processing }\end{array}$ & $\begin{array}{l}\text { Accelerating the boarding process can improve the capacity of transportation } \\
\text { network to a level equal or greater than the flow rate of traffic across the } \\
\text { border without any transportation construction. }\end{array}$ \\
\hline $\begin{array}{l}\text { Transportation Demand } \\
\text { Management }\end{array}$ & $\begin{array}{l}\text { It focuses on control the transportation demand by the optimal use of existing } \\
\text { and/or future infrastructure. Measures such as Intelligent Transportation } \\
\text { Systems (ITS) technologies and transportation/land use policies with } \\
\text { incentives to reduce, shift or divert transportation demand, may result in } \\
\text { deferring the need for expansion of the transportation network. }\end{array}$ \\
\hline $\begin{array}{l}\text { New and/or Improved } \\
\text { Rail Alternatives With } \\
\text { New or Expanded } \\
\text { International Crossing }\end{array}$ & $\begin{array}{l}\text { Part of international and regional goods transportation in this area is currently } \\
\text { carried out by rail. Improving the rail network either by adding a new railway } \\
\text { or expansion of existing crossings may decrease the truck traffic from the } \\
\text { road network and impact the need or timing of roadway-based improvements. }\end{array}$ \\
\hline $\begin{array}{l}\text { New and/or Improved } \\
\text { Transit and Marine } \\
\text { Services }\end{array}$ & $\begin{array}{l}\text { This alternative includes methods using the currently available crossing link } \\
\text { in the area such as introducing a new bus service through the Ambassador } \\
\text { bridge and/or expansion of existing bus service in the tunnel. Transit share of } \\
\text { the annual passenger cross-border trips at time of study (2000) was about } 2 \% \text {, } \\
\text { while only less than } 1 \% \text { of the international freight shipment served by } \\
\text { marine. Therefore, improvement/expansions of the capacity and/or service of } \\
\text { transit and marine services may reduce, shift or divert road-based passenger } \\
\text { and freight travel demand. }\end{array}$ \\
\hline $\begin{array}{l}\text { New and/or Improved } \\
\text { Road Alternatives With } \\
\text { New or Expanded } \\
\text { International Crossing }\end{array}$ & $\begin{array}{l}\text { Improvement the connecting between the highways network in } \\
\text { Detroit/Wayne to the Highway } 401 \text { in Windsor/Essex by new and/or } \\
\text { expansion of the international crossing to accommodate high volumes of } \\
\text { international and/or inter-regional long distance, traffic. The river crossing } \\
\text { could be either a new crossing (bridge or tunnel) or an expanded existing } \\
\text { crossing. For the purposes of this study, a second span at the Ambassador } \\
\text { Bridge crossing is considered to be an expansion of the existing crossing. } \\
\text { Converting a rail tunnel to accommodate vehicular traffic is considered to } \\
\text { provide a new crossing for road-based traffic. }\end{array}$ \\
\hline
\end{tabular}

Alternative 1 or "do nothing" was used as the base case to compare other alternatives with it. Alternatives 2, 3 and 4 were identified as temporary solutions while can be implemented fast. These alternatives may be consolidated and put forward as a 
transportation network improvement strategy to both expand the transportation network and reduce, shift or divert various aspects of travel demand. Since there is a need for higher capacity, a long-term solution should be one of the marine, rail or road alternatives. To demonstrate the application of the proposed framework these three longterm alternatives, i.e. road, rail and marine access, were considered as alternative solutions for the identified need. At this point, the first module of the framework is completed and its output is 3 alternatives for a new/expanded access between Detroit, Michigan and Winsdor, Ontario.

Next step is customizing the belief network based on features of this project.

\subsubsection{Bayesian Belief Model Customization for DRIC project}

As discussed in the previous section, DRIC is a bi-national large size transportation project, which has several stakeholders from both U.S. and Canada engaged in it. The decision-makers for selecting the best alternative needed to consider different aspects of the alternatives under consideration including but not limited to environmental impact, economic impact, project costs and benefits, and social impact. All of the factors in the generic belief network that affect the decision-making process were relevant in this case and offered an efficient-case scenario to perform a satisfactory case analysis.

The model customization begins with adaptation of the model factors and states of each factor with respect to the features and constraints of the specific project under consideration. Every major transportation project is unique and may involve various factors with different influence on decision-making process in the front-end phase of the project. It is important to notice that that the factors and accessible data related to these 
factors are not necessarily the same for all projects. Therefore, to ensure considering every specific future for the particular project under consideration, the user of the model should conduct a subjective assessment of the generic belief network.

\subsubsection{Decision Node Customization and Probabilities for DRIC Project}

As explained in past chapters, the decision nodes are the nodes in the lower level of the network and have only children. One step of model customization involves revision of the states for the decision nodes in the belief network for the specific project under consideration. This model enable the users of the model to evaluate possible combinations of the states of the five decision nodes to direct them towards selecting the optimal project alternative with respect to the project objectives and constraints. For example, in the early stage of DRIC project, different alternatives were made based on different transportation mode, without considering the specific location or corridor for the project. The decision to select the most suitable corridor was made later with more detail and only for the selected transportation mode. Thus, in the proposed belief network, the decision node regarding "corridor selection" is deleted for this project.

The conditional probabilities for the belief network analysis should be entered by the user of the model and then, the user may run the model only for the scenarios under consideration and continue to work with the results for the appropriate scenarios in the model. Table 6-2 shows the decision node states for three alternatives (scenarios).

Table 6-3: Modified decision nodes and related node states

\begin{tabular}{|l|c|c|c|}
\hline Decision node & \multicolumn{3}{|c|}{ State } \\
\hline Required level of service & A & B & C \\
\hline Transportation mode selection & Road & Rail & Marine \\
\hline Project start-up time & Less than 5 years & More than 5 years & - \\
\hline Project life time & Less than 20 years & 21 to 30 years & 31 to 50 years \\
\hline Contract type & Traditional & $\begin{array}{c}\text { Innovative } \\
\text { PPP is allowed }\end{array}$ & $\begin{array}{c}\text { Innovative } \\
\text { PPP is not allowed }\end{array}$ \\
\hline
\end{tabular}


The traffic level of service (LOS) is a measure that qualitatively describes the operating conditions of a transportation facility based on factors such as speed, travel time, maneuverability, delay, and safety. The level of service of a facility is designated with a letter, A to $\mathrm{F}$, with $\mathrm{A}$ representing the best operating conditions and $\mathrm{F}$ the worst. This factor has been identified as a decision node since the decision-makers often decide about future level of service of the proposed plan and it has a significant impact on the other factors such as safety and travel time. In this case, LOS is assumed to be B for both alternatives.

Transportation mode is basically the main distinctive between existing initial alternatives for this case. Therefore, it appears as a decision node and the road, rail or marine transportation input as observation with $100 \%$ prior probability. The other decision node is project start-up time, and it is important because it shows when the anticipated benefits of the project will be achieved. Two of three alternatives under consideration in this case are expected to start after more than 5 years (road and rail), unlike the marine, which is anticipated to start the operation in less than 5 years. All other temporary alternatives such as "border processing improvement" or "transportation demand management" are also categorized as alternatives with start-up less than 5 years.

In addition, decision about the expected lifetime of a project can be one of the decision nodes. In this case, rail alternative is expected to be a long-term project, so the state "31 to 50 years" has been selected in lifetime decision node. But road and marine alternatives defined as mid-life projects, or "21 to 30 year".

Contract type is an important factor and plays significant role in project delivery and financing model. As discussed in chapter 3 there are several contract types for 
transportation projects, not all of them allowed by the regulations in all U.S. states. Especially in major projects, it is important whether a public-private-partnership contract is allowed or not and if any modern contracts are acceptable. In this case all three alternatives can follow with innovative-PPP allowed.

Several scenarios can be identified from combination of different states of decision nodes, but not all of them are appropriate and practical alternatives for this project. Among all possible scenarios based on all states of decision nodes discussed above, 3 scenarios were developed to run in AgenaRisk for three suggested alternatives. The description of the scenarios is summarized in Table 6-4.

Table 6-4: DRIC project alternatives- BBN scenarios

\begin{tabular}{|l|c|c|c|l|l|}
\hline & $\begin{array}{l}\text { Transportation } \\
\text { mode }\end{array}$ & $\begin{array}{l}\text { Level of } \\
\text { service }\end{array}$ & $\begin{array}{l}\text { Project start-up } \\
\text { time }\end{array}$ & $\begin{array}{l}\text { Project } \\
\text { lifetime }\end{array}$ & Contract type \\
\hline Alternative 1 & Road & A & $>5 y r s$ & $21-30 y r s$ & $\begin{array}{l}\text { Innovative- } \\
\text { PPP is Allowed }\end{array}$ \\
\hline Alternative 2 & Rail & A & $>5 y r s$ & $31-50 y r s$ & $\begin{array}{l}\text { Innovative- } \\
\text { PPP is Allowed }\end{array}$ \\
\hline Alternative 3 & Marine & A & $<5 y r s$ & $21-30 y r s$ & $\begin{array}{l}\text { Innovative- } \\
\text { PPP is Allowed }\end{array}$ \\
\hline
\end{tabular}

After reviewing all starting and intermediate nodes in the proposed generic network, the nodes consistent with features of this project are kept or modified, and the irrelevant factors were removed. For example, as discussed earlier, corridor selection node is removed in this stage of decision making for DRIC project. Therefore, any predefined relation between that node and other existing nodes were removed.

Next step in development of Bayesian belief network is quantification of the relationships and input the values for prior and conditional probabilities into the crated network, which is typically the most difficult part in belief-network development. Generally, this step can be carried out in two different methods. If the belief network is 
related to an area where the relationship between the factors and variables remain constant in different applications, available data and statistical approaches for repeated events (i.e. historic data) can be used in capturing the relationships in the belief network. For example, this method is used extensively in medicine to detect presence or absence of disease. To construct a belief network for diagnosis of diseases, several sources of data can be used, such as different medical tests, and other methods that result in similar diagnosis for different patients with the same symptom and condition. In such case, relationships between factors can be identified based on the available data and if any changes happen the user of the model should only update the network and relationships.

However, in many other cases such as this research with unique features, constraints, and conditions in every single project, a typical quantification of the relationships is not feasible. The belief network for this research is unique for any single MTP due to its features. Although conceived information from review of past studies can be helpful in defining the probabilistic relations and values, the majority of these values should be entered by the model user for the specific project under consideration. In this method of qualification of belief network relations, opinion of project experts are very fundamental in development and correctness of the model (Bayraktar and Hastak 2009).

To identify each alternative for the project, the user of the model selects a state for each decision node (absolute observation). Based on the definition of decision nodes, the prior probability of the selected state becomes $\% 100$, while the probabilities of the other states of that particular node are forced down to $\% 0$. Therefore, the importance of prior probabilities assigned to the states of the decision nodes is mostly reveled in evaluating different "what-if?" scenarios. It is important to note that equal weights were assigned to 
the states of each decision node since the user of the model has absolute control over the decision process and the states of the decision nodes present the same likelihood to be selected by the user as he or she does not have any preference for any state over the other states of the same decision node. State probability table of decision nodes is presented in Table 6-5.

Table 6-5: Decision node probability table for DRIC project

\begin{tabular}{|c|c|c|c|c|c|}
\hline Decision node & States & $\begin{array}{l}\text { Prior } \\
\text { Probability }\end{array}$ & $\begin{array}{l}\text { Probability for } \\
\text { Alternative } 1 \\
\end{array}$ & $\begin{array}{l}\text { Probability for } \\
\text { Alternative } 2 \\
\end{array}$ & $\begin{array}{l}\text { Probability for } \\
\text { Alternative } 3 \\
\end{array}$ \\
\hline \multirow{3}{*}{$\begin{array}{l}\text { Required level } \\
\text { of service }\end{array}$} & A & 0.33 & 1 & 1 & 1 \\
\hline & $\mathrm{B}$ & 0.33 & 0 & 0 & 0 \\
\hline & $\mathrm{C}$ & 0.33 & 0 & 0 & 0 \\
\hline \multirow{3}{*}{$\begin{array}{l}\text { Transportation } \\
\text { mode selection }\end{array}$} & Road & 0.33 & 1 & 0 & 0 \\
\hline & Rail & 0.33 & 0 & 1 & 0 \\
\hline & Marine & 0.33 & 0 & 0 & 1 \\
\hline \multirow{2}{*}{$\begin{array}{l}\text { Project start- } \\
\text { up time }\end{array}$} & $>5 y r s$ & 0.5 & 1 & 1 & 0 \\
\hline & $<5 y r s$ & 0.5 & 0 & 0 & 1 \\
\hline \multirow{3}{*}{$\begin{array}{l}\text { Project life } \\
\text { time }\end{array}$} & $<20 y r s$ & 0.33 & 0 & 0 & 0 \\
\hline & 21-30yrs & 0.33 & 1 & 0 & 1 \\
\hline & $31-50 y r s$ & 0.33 & 0 & 1 & 0 \\
\hline \multirow[t]{3}{*}{ Contract type } & Traditional & 0.33 & 0 & 0 & 0 \\
\hline & $\begin{array}{l}\text { Innovative- } \\
\text { PPP is allowed }\end{array}$ & 0.33 & 1 & 1 & 1 \\
\hline & $\begin{array}{l}\text { Innovative- } \\
\text { PPP is not } \\
\text { allowed }\end{array}$ & 0.33 & 0 & 0 & 0 \\
\hline
\end{tabular}

\subsubsection{Starting Node Prior Probabilities for DRIC Project}

As discussed in Chapter 5, the starting nodes are nodes without parents and are only influenced by the external factors such as the inflation rate. The belief network is exposed to external factors only by these nodes. As discussed earlier, there are some factors not shown in the influence pattern but may have effects on decision indicators through the starting nodes. Such effects are synthetized in the prior probabilities of the starting nodes as appropriate and will be reflected further in the children nodes of the starting nodes through conditional probabilities. 
Table 6-6 shows the starting node prior probabilities for DRIC project. The probabilities of the states of each starting node should add up to one.

Table 6-6: Starting node probability table for DRIC project

\begin{tabular}{|l|l|c|}
\hline Starting node & State & Prior probability \\
\hline \multirow{3}{*}{ Business productivity } & Improve & 0.5 \\
\cline { 2 - 3 } & No change & 0.35 \\
\cline { 2 - 3 } & Decline & 0.15 \\
\hline \multirow{3}{*}{ Convention business } & Improve & 0.5 \\
\cline { 2 - 3 } & No change & 0.3 \\
\cline { 2 - 3 } & Decline & 0.2 \\
\hline \multirow{2}{*}{ Extending geographic market } & Vast & 0.2 \\
\cline { 2 - 3 } & Moderately & 0.8 \\
\hline \multirow{3}{*}{ Industrial diversification } & High & 0.8 \\
\cline { 2 - 3 } & Low & 0.4 \\
\hline \multirow{3}{*}{$\begin{array}{l}\text { Relocation of } \\
\text { residents/businesses }\end{array}$} & Increase & 0.7 \\
\cline { 2 - 3 } $\begin{array}{l}\text { Contribution to development } \\
\text { strategies }\end{array}$ & No change & 0.25 \\
\cline { 2 - 3 } & Decrease & 0.05 \\
\cline { 2 - 3 } & Extremely & 0.6 \\
\hline \multirow{2}{*}{ Visual impacts } & Moderately & 0.4 \\
\cline { 2 - 3 } & Ineffective & 0.7 \\
\hline \multirow{2}{*}{ Project location } & Positive & 0.3 \\
\cline { 2 - 3 } & Negative & 0.5 \\
\hline & Accessible & 0.5 \\
\cline { 2 - 3 } & Inaccessible & 0.3 \\
\hline
\end{tabular}

\subsubsection{Intermediate Node Conditional Probabilities for DRIC Project}

Intermediate nodes are the mid level nodes in the belief network with both parents and children. The conditional probabilities of the states of that particular node are expressed in an associated node probability table for each intermediate node. The table includes all possible combinations of states for the node parents. In this step of the model, the model user is asked to provide the values required for the node probability tables of the intermediate nodes considering the perceived probabilistic node outcomes associated with the expected project conditions. 


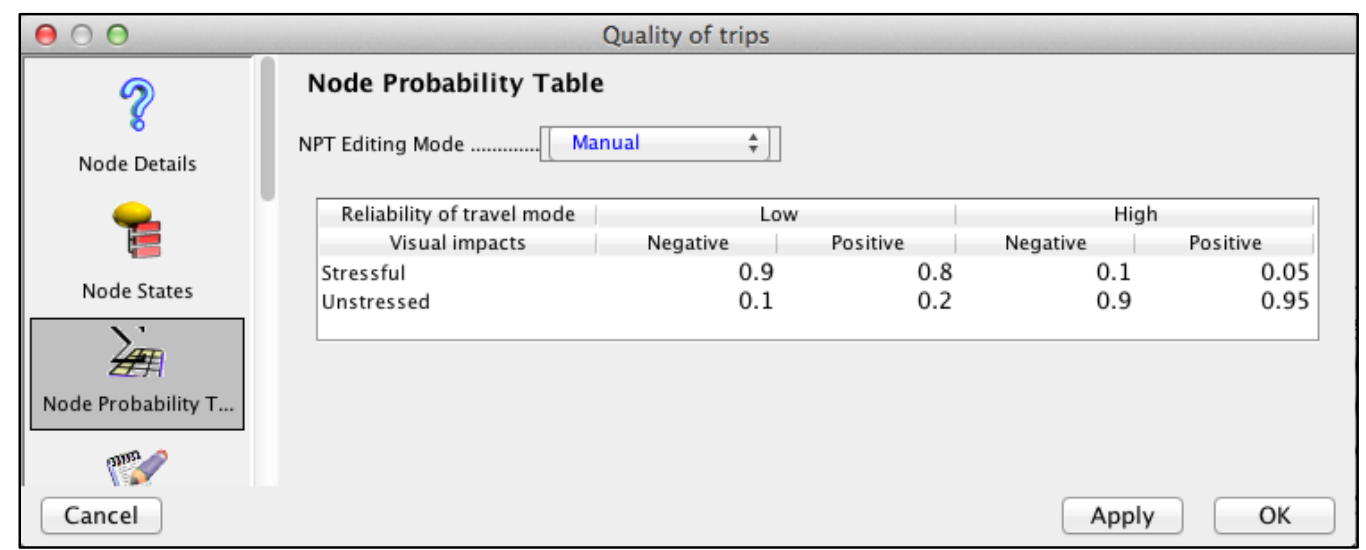

Figure 6-10: Node probability table for the "Quality of trips" node of DRIC project

Figure 6-10 illustrates the node probability table of the "Quality of trips" node used for the analysis of DRIC project. All of the intermediate node conditional probability tables for this project are available and can be presented by the author if needed.

\subsubsection{Target Node Conditional Probabilities for DRIC Project}

For the purposes of this research, target nodes were defined as the nodes, which only have parent nodes in the belief network. There are seven target nodes in the belief network proposed for major transportation projects: (i) Economic development, (ii) Social development, (iii) Protect natural environmental, (iv) Transportation benefits, (v) Technical feasibility, (vi) Project cost, and (vii) Financial feasibility. Type of each of these target nodes selected as "Rank" in the AgenaRisk that assigns five states including: (i) very low, (ii) low, (iii) medium, (iv) high, and (v) very high. The target node conditional probability tables for DRIC project are available and can be presented by the author if needed.

\subsubsection{Target Node State Probability Values DRIC Project}

To achieve the final objective of belief network, the last step after the completion of quantification of the relationships is the belief network is ready to. Therefore, once the starting node probabilities are entered and the intermediate node and target node 
conditional probability tables are established by the user, the belief network is ready to calculate the state probability values for the target nodes, i.e., economic development, social development, protect natural environmental, transportation benefits, technical feasibility, project cost, and financial feasibility. For the analysis of DRIC project, three scenarios were defined based on three alternatives and the belief network was run to receive the probabilities for the three alternatives, as shown in Table 6-7. 
Table 6-7: Node state probability of decision indicators for 3 alternatives

\begin{tabular}{|c|c|c|c|c|c|c|c|c|c|c|c|c|c|c|c|}
\hline \multirow{2}{*}{$\begin{array}{l}\text { Decision } \\
\text { Indicator }\end{array}$} & \multicolumn{5}{|c|}{ Alternative 1: Road } & \multicolumn{5}{|c|}{ Alternative 2: Rail } & \multicolumn{5}{|c|}{ Alternative 3: Marine } \\
\hline & Very low & Low & Medium & High & $\begin{array}{l}\text { Very } \\
\text { high }\end{array}$ & Very low & Low & Medium & High & $\begin{array}{l}\text { Very } \\
\text { high } \\
\end{array}$ & Very low & Low & Medium & High & $\begin{array}{l}\text { Very } \\
\text { high }\end{array}$ \\
\hline $\begin{array}{c}\text { Economic } \\
\text { development }\end{array}$ & 4.343 & 13.605 & 25.616 & 39.335 & 17.101 & 5.064 & 15.564 & 25.762 & 36.494 & 17.117 & 6.083 & 18.061 & 26.685 & 33.846 & 15.325 \\
\hline $\begin{array}{c}\text { Social } \\
\text { development }\end{array}$ & 7.452 & 16.278 & 39.231 & 24.646 & 12.393 & 8.093 & 18.074 & 41.04 & 22.591 & 10.202 & 8.29 & 18.938 & 42.021 & 21.691 & 9.06 \\
\hline $\begin{array}{l}\text { Protect natural } \\
\text { environment }\end{array}$ & 7.651 & 19.779 & 34.806 & 26.052 & 11.712 & 9.154 & 22.808 & 43.818 & 19.236 & 4.983 & 6.494 & 17.909 & 35.001 & 27.953 & 12.644 \\
\hline $\begin{array}{c}\text { Transportation } \\
\text { benefits }\end{array}$ & 3.324 & 8.107 & 26.505 & 39.625 & 22.438 & 2.263 & 5.543 & 24.802 & 42.694 & 24.921 & 3.727 & 8.713 & 26.963 & 38.676 & 21.921 \\
\hline $\begin{array}{l}\text { Technical } \\
\text { feasibility }\end{array}$ & 16.634 & 30.74 & 30.80 & 14.559 & 7.267 & 11.485 & 24.787 & 34.298 & 19.342 & 10.088 & 17.198 & 30.656 & 29.136 & 15.421 & 7.589 \\
\hline Project cost & 1.737 & 15.842 & 33.357 & 45.694 & 3.369 & 1.471 & 14.318 & 30.442 & 50.077 & 3.692 & 1.61 & 14.832 & 30.267 & 49.243 & 4.049 \\
\hline $\begin{array}{l}\text { Financial } \\
\text { feasibility }\end{array}$ & 2.931 & 22.062 & 25.571 & 46.861 & 2.576 & 3.251 & 24.723 & 26.457 & 43.253 & 2.315 & 4.021 & 26.08 & 27.223 & 40.706 & 1.969 \\
\hline
\end{tabular}


Figures 6-11 through 6-17 provide a visual comparison of the state probabilities for the three alternative contracting strategies (Table 6-7) with respect to the seven decision indicators, i.e., economic development, social development, protect natural environmental, transportation benefits, technical feasibility, project cost, and financial feasibility.

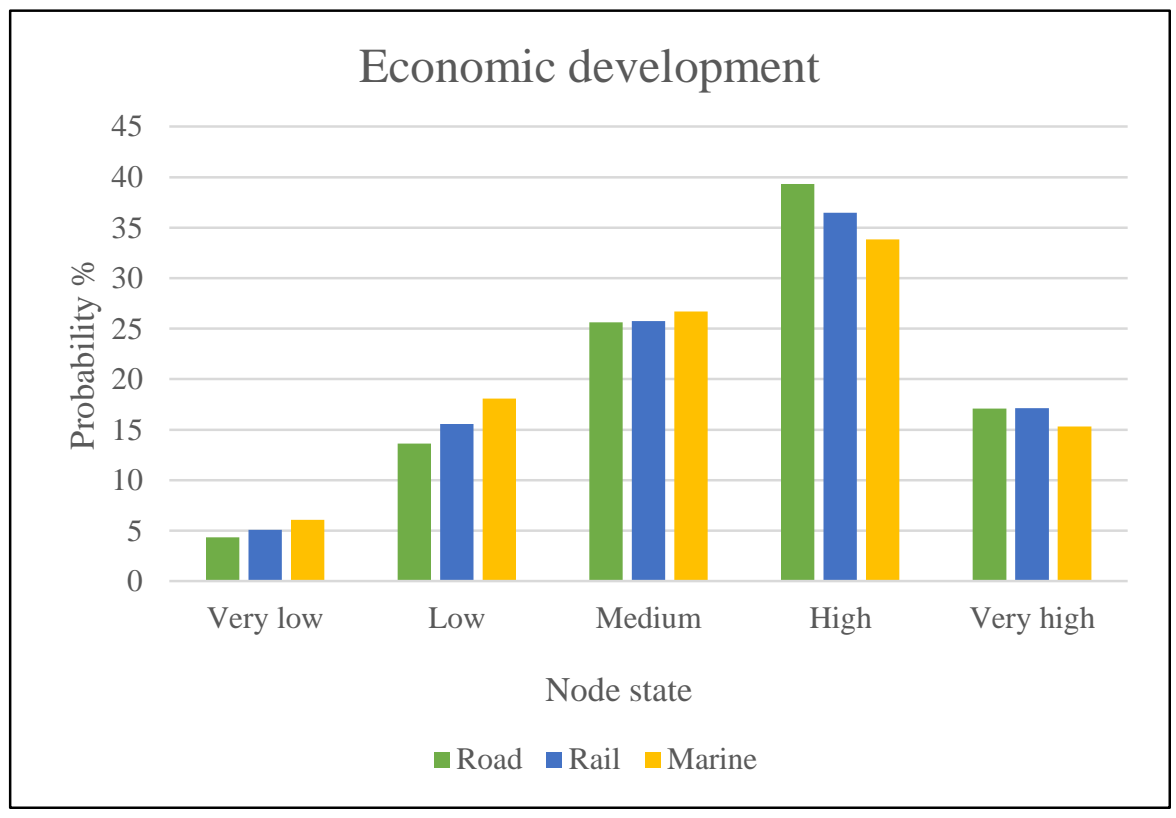

Figure 6-11: Economic development state probabilities for DRIC project 


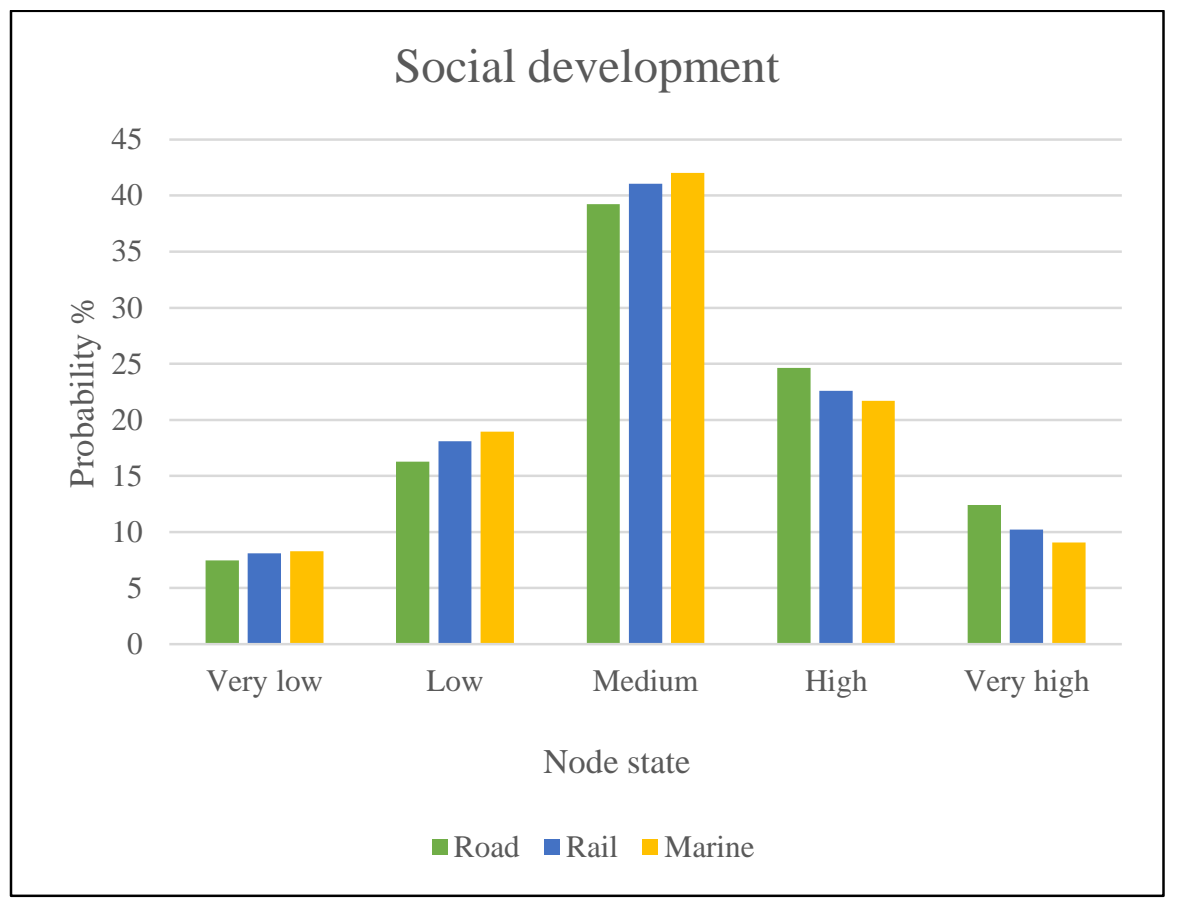

Figure 6-12: Social development probability state of DRIC project

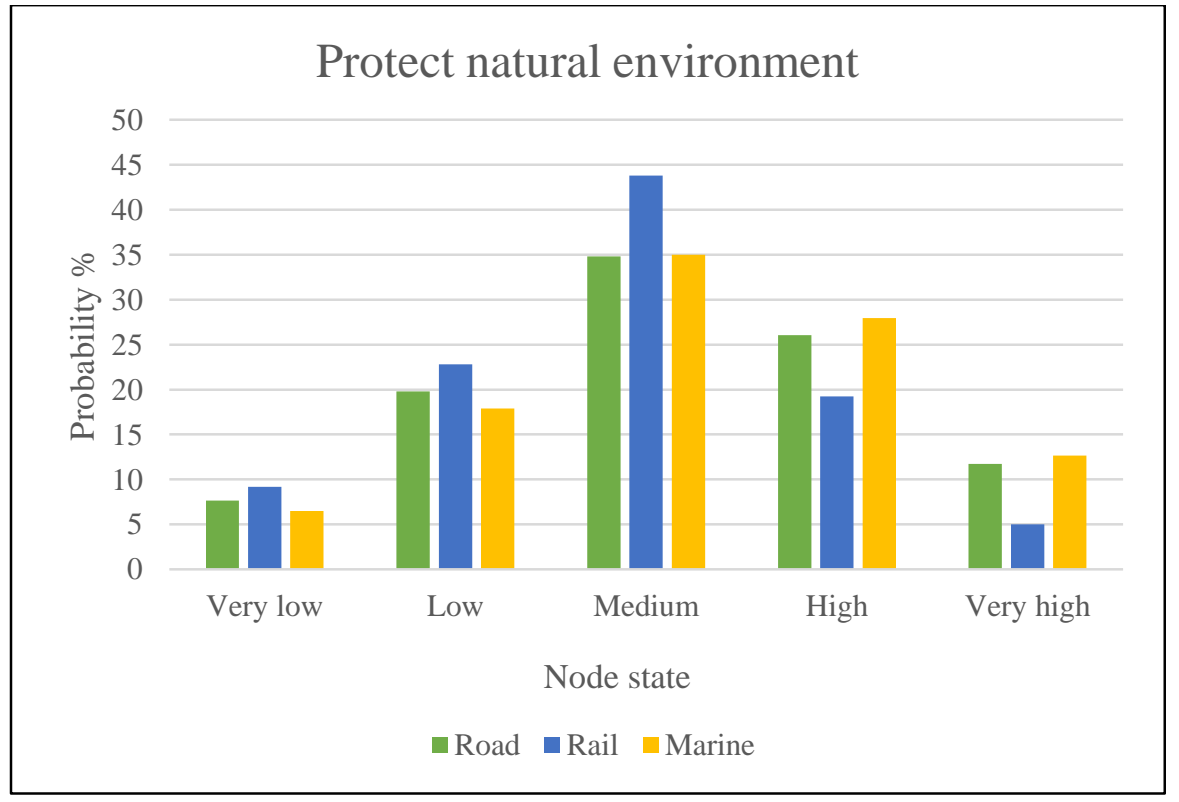

Figure 6-13: Protect natural environment state probabilities for DRIC project 


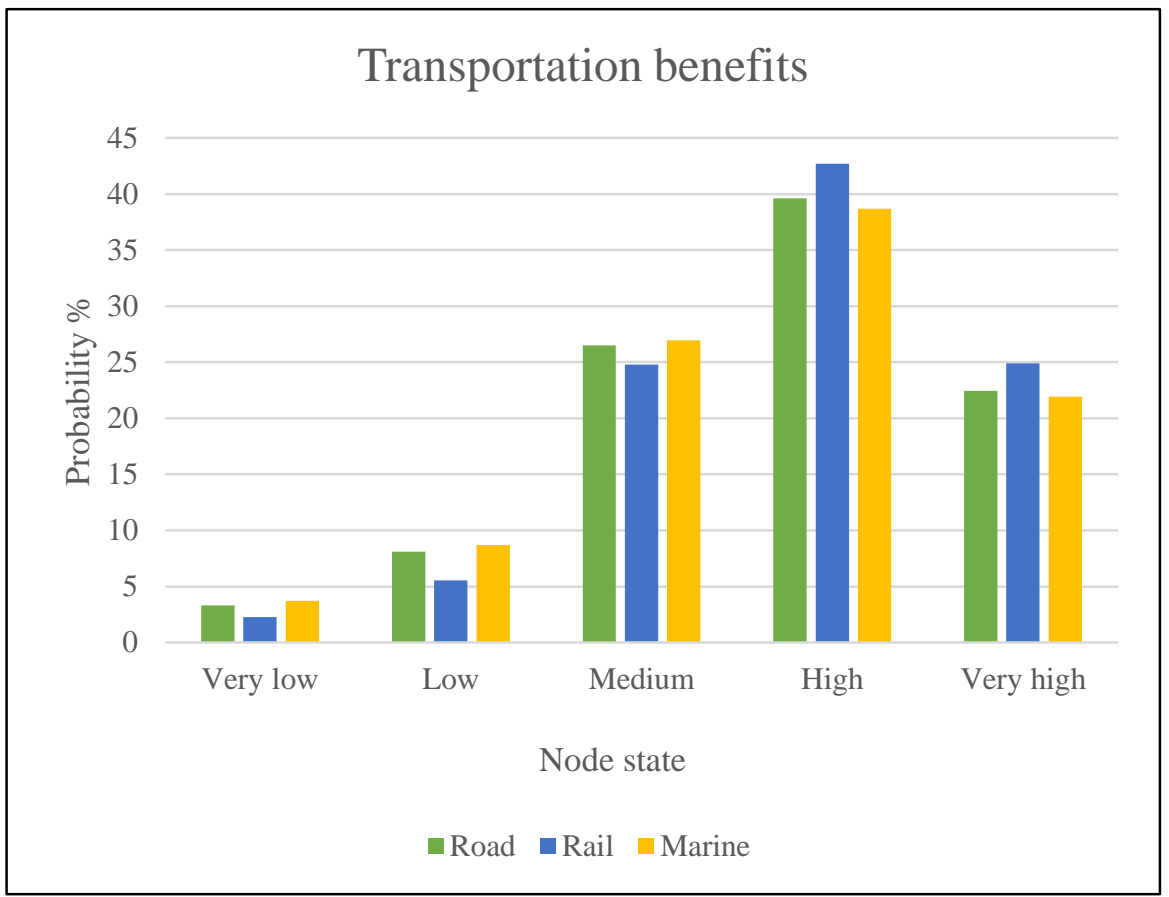

Figure 6-14: Transportation benefits state probability of DRIC project

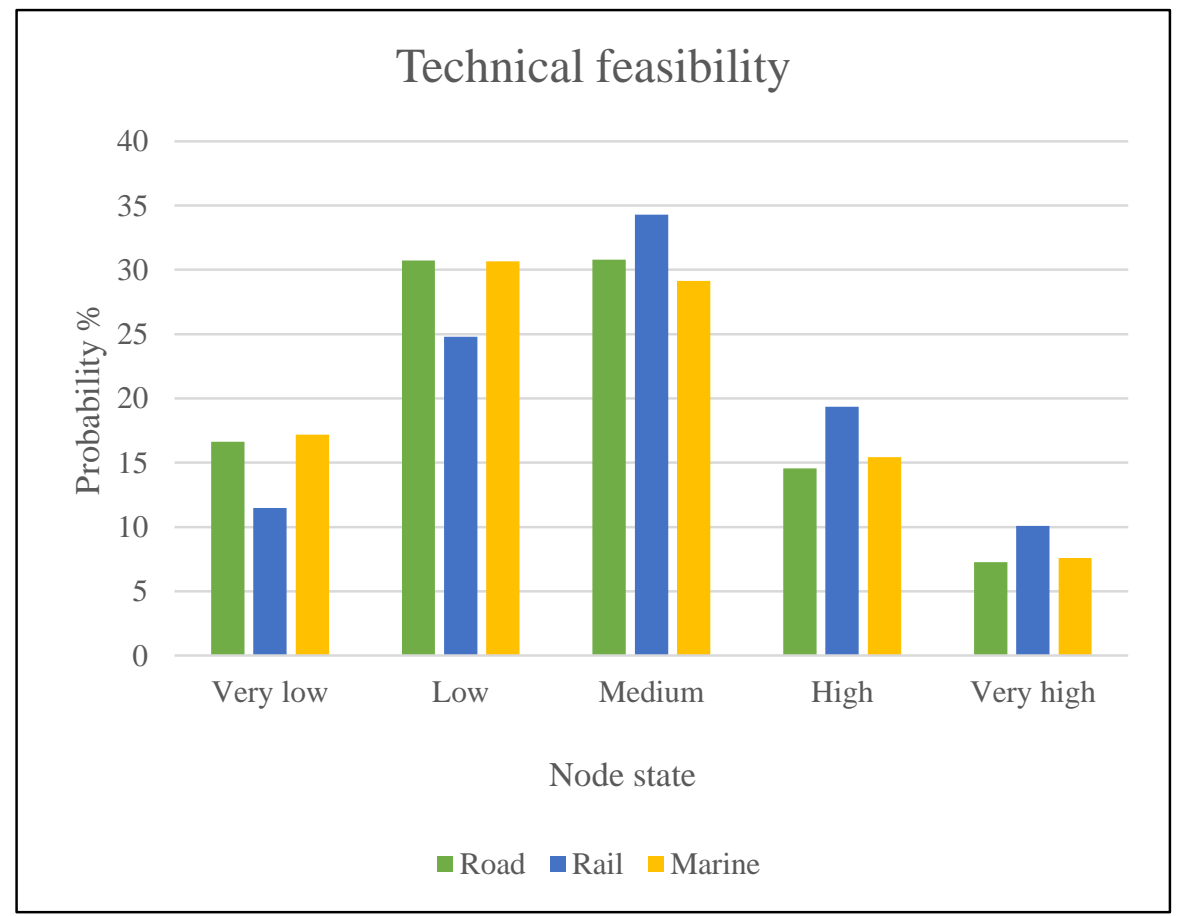

Figure 6-15: Technical feasibility state probability for DRIC project 


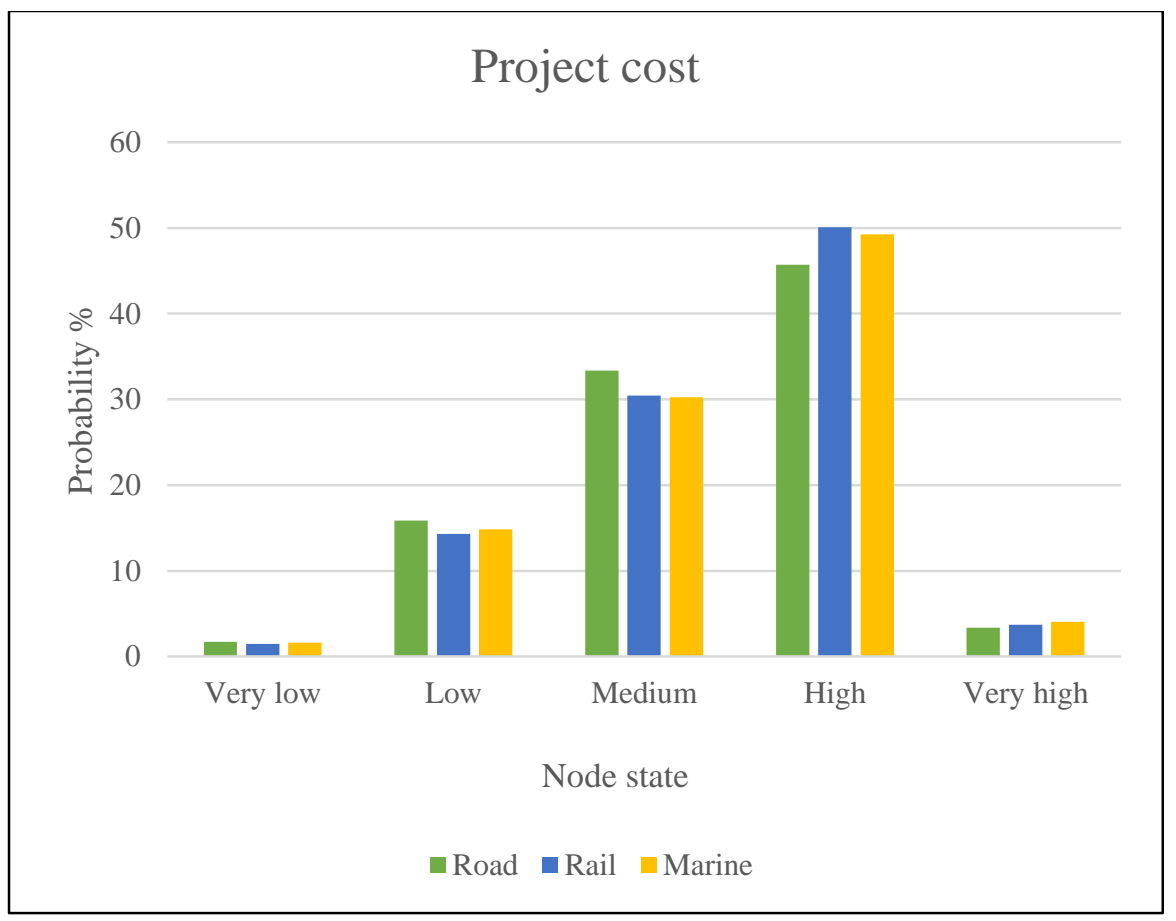

Figure 6-16: Project cost state probability for DRIC project

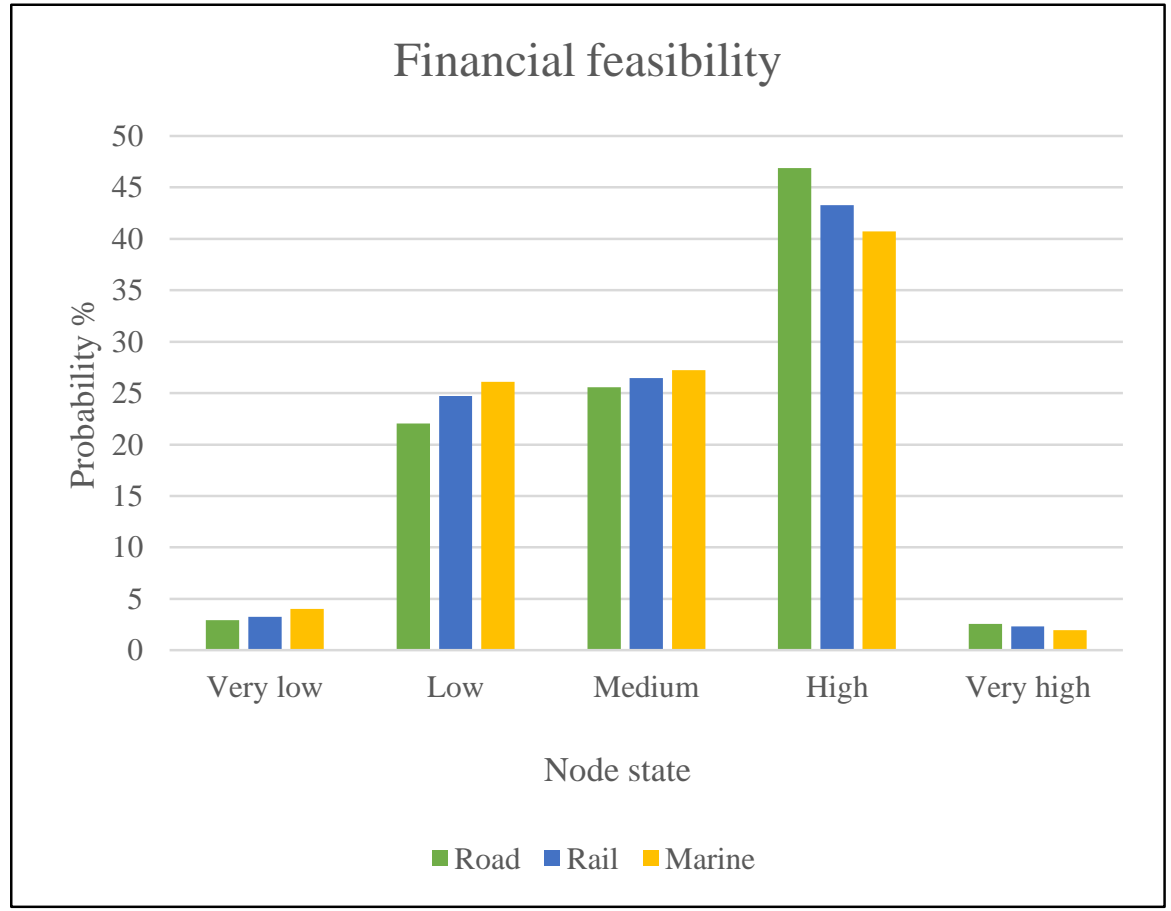

Figure 6-17: Financial feasibility state probability for DRIC project 
The output of BBN model reveals that there was no major difference between the three alternatives with respect to most of the decision indicators. However, for the "protect natural environmental" and the "Technical feasibility" decision indicators the results were slightly different. This information cannot identify which alternative is better in this stage. Having these probability distributions, the user of the model will be able to implement the third module. The application of the third module is explained thoroughly in the next section.

\subsubsection{Application of Alternative Selection Module for DRIC project}

In previous step of model application the user was able to calculate the target node state probabilities of the seven decision indicators (Table 6-7) for the suggested three project alternatives (Table 6-4). The graphs illustrating the same information separately for each of the seven decision indicators helped the user have better idea about the different aspects of individual alternatives. In this step, the model attempts to rank the proposed alternatives with respect to the seven decision indicators based on the stakeholders' preferences for the particular MTP under consideration. Therefore it requires identifying the key stakeholders' of the project who play significant role in decision-making process in the front-end phase of this particular project (DRIC project). After searching among project history and published document as well as interview with project team coordinators, three major stakeholders were identified for DRIC project.

The first and major stakeholder is a joint authority including Michigan Department of Transportation (MnDOT) and Ministry of Transportation of Ontario (MTO) that played the leading role in project implementation. In the early stage decisionmaking these two agencies could be assumed as a single stakeholder due to the similar 
objectives in the project. However, in the next stage, which was selecting the best corridor and location for the project, they should be count as two separate stakeholders with conflict of interests. This stakeholder is called MnDOT/MTO in this study.

Another significant identified stakeholder is community or public whose satisfaction was very important in the entire process of decision-making of this particular project. The project team held numerous public hearing sessions and conducted onsite questionnaire studies to ensure about public engagement in the final decision-making. Therefore, in application of this framework for DRIC project Public is included as another stakeholder for the alternative selection module.

Similar to any major project, the financer of project is another important stakeholder and is included in this model application. The project was supposed to finance by both governments from U.S. and Canada, however, the Michigan state senate did not approve the budget and finally it ended up by financing with Canadian side only.

Reviewing the scoping documents and public hearing documents and project reports helped to configure the constraints and preferences of these stakeholders in selecting the project alternative. Phone interviews were also conducted with two members of project team from MnDOT to get information about project and stakeholders. The stakeholders' satisfaction threshold matrix for DRIC project is presented in Table 6-8.

After identifying the constraints and rules, the VB code for Monte Carlo simulation model was adjusted to find if a random set of BBN output, i.e., a ransom set of decision indicator states for each alternative, is satisfactory for each individual stakeholder or not. A matrix of stakeholders' satisfaction was obtained for every random 
iteration. The ratio of satisfactory iteration to total iteration shows satisfaction level of each alternative with respect to different stakeholders.

Table 6-8: Stakeholders' satisfaction threshold matrix for DRIC project

\begin{tabular}{|l|l|l|l|}
\hline \multirow{2}{*}{ Decision indicator } & \multicolumn{3}{|l|}{ Satisfaction threshold } \\
\cline { 2 - 4 } & MnDOT/MTO & Public/Community & Financer \\
\hline Economic development & Medium or higher & Medium or higher & Medium or higher \\
\hline Social development & Medium or higher & High or very high & Low or higher \\
\hline $\begin{array}{l}\text { Protect natural } \\
\text { environment }\end{array}$ & Medium or higher & High or very high & Medium or higher \\
\hline Transportation benefits & Medium or higher & Medium or higher & - \\
\hline Project cost & Medium or higher & - & - \\
\hline Technical feasibility & Medium or less & - & Medium or less \\
\hline Financial feasibility & Low or higher & - & High or higher \\
\hline
\end{tabular}

The Monte Carlo simulation model was run three times for each alternative with 100, 200, 1000 and 1500 iteration. Since the results for alternative 1 and alternative 2 were convergent, the model run was stopped for these two alternatives. But fluctuation of the results of Monte Carlo simulation for alternative 3 (Marine) was higher; therefore, another run with 3000 iterations was run. The stochastic results of Monte Carlo simulation model are summarized in Table 6-9.

The numbers in above Table 6-9 show what is the likelihood of each alternative to be satisfactory by individual stakeholders and all of them together in the same time in each run of the Monte Carlo simulation. For example, the value $14 \%$ in the top left cell means that in 100 random scenarios of different states of decision indicators for alternative 1, in 14 cases the alternative meets the entire constraints of MnDOT/MTO. 
Table 6-9: Percentage of satisfactory runs of each alternative in respect to stakeholders

\begin{tabular}{|c|c|c|c|c|c|}
\hline \multicolumn{2}{|c|}{ \# of iterations } & MnDOT/MTO & Public/Community & Financer & All \\
\hline \multirow{4}{*}{ 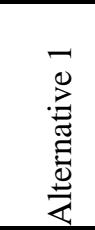 } & 100 & 14 & 61 & 37 & 10 \\
\hline & 200 & 14.5 & 60 & 32.5 & 10 \\
\hline & 1000 & 13.1 & 59.8 & 34.7 & 10.2 \\
\hline & 1500 & 13.47 & 60.1 & 33.93 & 10.1 \\
\hline \multirow{4}{*}{ 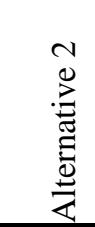 } & 100 & 13.2 & 64 & 27.3 & 9.2 \\
\hline & 200 & 15 & 60.5 & 26.5 & 9.5 \\
\hline & 1000 & 13.7 & 58.1 & 26 & 9.6 \\
\hline & 1500 & 14.2 & 59.1 & 29.27 & 10.3 \\
\hline \multirow{5}{*}{ 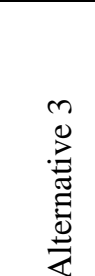 } & 100 & 13 & 60 & 21 & 8 \\
\hline & 200 & 8 & 55.5 & 27 & 4 \\
\hline & 1000 & 9.1 & 57.2 & 26.9 & 5.6 \\
\hline & 1500 & 10.6 & 55.07 & 27.8 & 8.07 \\
\hline & 3000 & 10.53 & 56 & 27.93 & 7.33 \\
\hline
\end{tabular}

At the final step of model, the Alternative Desirability Index (ADI) is calculated to select the most desirable alternative based on multi decision-makers satisfaction. As explained in chapter 5, ADI can be obtained as follows:

$$
A D I=\sum_{i=1}^{n} w_{i} P_{S_{i}}
$$

where $n$ is number of the stakeholders, $w_{i}$ represents the weight of each stakeholder's impact in decision-making, $P_{S i}$ is the likelihood of an alternative to be desirable or satisfactory for any individual stakeholder $\left(S_{i}\right)$. Value for the alternative desirability index is between 0 and 1, which higher value indicates a better alternative. In this application equal weights were assumed for three stakeholders. It can be changed by 
the user of the model based on condition of the user of the model. The calculated ADI for DRIC project alternatives are presented in Table 6-10.

Table 6-10: Alternative desirability index of three alternatives for DRIC project

\begin{tabular}{|c|c|c|c|c|}
\hline & $\mathrm{S}_{1}$ & $\mathrm{~S}_{2}$ & $\mathrm{~S}_{3}$ & $\mathrm{ADI}_{\mathrm{i}}$ \\
\hline Alternative 1 & 0.1347 & 0.601 & 0.3393 & 0.35475 \\
\hline Alternative 2 & 0.142 & 0.591 & 0.2927 & 0.338481 \\
\hline Alternative 31 & 0.1053 & 0.56 & 0.2793 & 0.311718 \\
\hline
\end{tabular}

According to obtained ADI for each alternative, it can be concluded that the most desirable alternative for the project under consideration will be alternative 1 which was a road alternative.

\subsection{Framework Validation}

The validation of the create framework have been done in 2 steps. First, the finding of the model was compared with real result of decision-making process of DRIC project. Then, project team members were contacted and were asked about applicability and validity of the model.

The project team established 6 evaluation factors to obtain the objectives of the planning/need and feasibility study. The evaluation also considers the consistency of the alternatives with environmental approval processes in both Canada and the U.S. The factors developed for evaluating the practicality and feasibility of transportation alternatives includes: 1) Transportation Network Improvement; 2) Transportation 
Opportunities; 3) Governmental Land Use, Transportation Planning and Tourism

\author{
Objectives; 4) Border Processing;5) Environmental Feasibility; and; 6) Technical
}

Feasibility. Although the rational and method of assessment for each factor is provided in

the feasibility report, the assessment was in macro level and many details were not

considered in the reported results. The decision-makers at this stage

The rationale and method of assessment used in the evaluation are listed in Table

6-11.

\title{
Table 6-11: Rationale and method of assessment used in the evaluation (FHWA et al. 2004)
}

\begin{tabular}{|c|c|c|}
\hline Factor & Rationale & Method of Assessment \\
\hline $\begin{array}{l}\text { Transportation } \\
\text { Network } \\
\text { Improvement }\end{array}$ & $\begin{array}{l}\text { Alternative would be considered feasible only } \\
\text { if it enhances the performance of the } \\
\text { transportation system with respect to the } \\
\text { quality of travel as defined by travel time, } \\
\text { travel speed, delay and reliability during the } \\
\text { planning horizon of this study (to 2030). } \\
\text { Improvements to transportation efficiency may } \\
\text { be gained by improving the utility of inefficient } \\
\text { or underutilized transportation corridors as well } \\
\text { as making use of planned network } \\
\text { improvements }\end{array}$ & $\begin{array}{l}\text { Assessment of ability of the } \\
\text { alternative to address congestion } \\
\text { on the transportation network by } \\
\text { improving travel time and } \\
\text { reliability for international } \\
\text { passenger and freight movement } \\
\text { Assessment of the ability of the } \\
\text { alternative to optimize use of } \\
\text { existing transportation corridors } \\
\text { or planned network } \\
\text { improvements }\end{array}$ \\
\hline $\begin{array}{l}\text { Governmental } \\
\text { Land Use, } \\
\text { Transportation } \\
\text { Planning and } \\
\text { Tourism Objectives }\end{array}$ & $\begin{array}{l}\text { Recognizing the importance and impacts of } \\
\text { accommodating the free flow of international } \\
\text { passengers and goods, consideration must be } \\
\text { given to the degree to which alternatives } \\
\text { support local, regional, provincial, state and } \\
\text { national planning and tourism objectives. }\end{array}$ & $\begin{array}{l}\text { Assessment of the degree to } \\
\text { which the alternative is } \\
\text { consistent with governmental } \\
\text { land use, transportation planning } \\
\text { and tourism objectives. }\end{array}$ \\
\hline Border Processing & $\begin{array}{l}\text { Alternatives would be considered feasible only } \\
\text { if the long-term needs of the U.S. and Canadian } \\
\text { border processing agencies can be met. }\end{array}$ & $\begin{array}{l}\text { Assessment of the ability of the } \\
\text { alternative to meet long-term } \\
\text { needs of border processing } \\
\text { agencies. }\end{array}$ \\
\hline $\begin{array}{l}\text { Environmental } \\
\text { Feasibility }\end{array}$ & $\begin{array}{l}\text { Consideration of potential impacts to } \\
\text { environmental constraints (including natural, } \\
\text { social and cultural features) is required under } \\
\text { the environmental approval processes in both } \\
\text { Canada and the U.S. }\end{array}$ & $\begin{array}{l}\text { Assessment as to whether } \\
\text { environmental constraints in the } \\
\text { FAA (including natural, social } \\
\text { and cultural features) preclude } \\
\text { the alternative. }\end{array}$ \\
\hline $\begin{array}{l}\text { Technical } \\
\text { Feasibility }\end{array}$ & $\begin{array}{l}\text { Alternatives requiring new or expanded } \\
\text { facilities would be considered feasible only if } \\
\text { technical requirements related to alignment } \\
\text { (both horizontal and vertical) and cross- section } \\
\text { can be achieved at a reasonable cost. }\end{array}$ & $\begin{array}{l}\text { Assessment of the ability of } \\
\text { alternative requiring new or } \\
\text { expanded facilities to achieve } \\
\text { minimum technical requirements } \\
\text { at a reasonable } \\
\text { construction/implementation } \\
\text { cost. }\end{array}$ \\
\hline
\end{tabular}


The result of the evaluation of transportation alternatives is summarized in graphic form in Table 6-12.

Table 6-12: Summary of evaluation of transportation alternatives (FHWA et al. 2004)

\begin{tabular}{|c|c|c|c|c|c|c|c|}
\hline Factor & $\begin{array}{l}\text { Do } \\
\text { Nothing }\end{array}$ & $\begin{array}{c}\text { Border } \\
\text { Processing }\end{array}$ & TDM & $\begin{array}{l}\text { Rail } \\
\text { Improvements }\end{array}$ & $\begin{array}{c}\text { Transit } \\
\text { Improvements }\end{array}$ & $\begin{array}{c}\text { Marine } \\
\text { Improvements }\end{array}$ & $\begin{array}{l}\text { New and/or } \\
\text { Expanded } \\
\text { Roadways }\end{array}$ \\
\hline \multicolumn{8}{|l|}{$\begin{array}{l}\text { Transportation Network } \\
\text { Improvement }\end{array}$} \\
\hline \multicolumn{8}{|l|}{$\begin{array}{l}\text { Transportation } \\
\text { Opportunities }\end{array}$} \\
\hline \multicolumn{8}{|c|}{$\begin{array}{l}\text { Governmental Land Use, } \\
\text { Transportation Planning } \\
\text { and Tourism Objectives }\end{array}$} \\
\hline \multicolumn{8}{|l|}{ Border Processing } \\
\hline \multicolumn{8}{|c|}{ Environmental Feasibility } \\
\hline Technical Feasibility & $\mathrm{N} / \mathrm{A}$ & & & & & & \\
\hline
\end{tabular}

Shading represents the degree to which the alternative addresses each factor, relative to the other alternatives

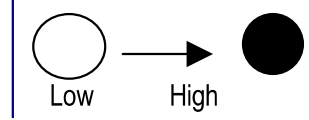

The selected alternative by project team was road alternative, which matches to the findings of this study.

\subsubsection{Sensitivity Analysis of the Bayesian Belief Network}

To identify the most important factors that have significant impact on each decision indicators, a sensitivity analysis was conducted for each target node of the BBN using AgenaRisk software. Ten top factors for each decision indicators are shown as the main influencing factors in the Table 6-13. 
Table 6-13: Most influencing factors of decision indicators for DRIC project

\begin{tabular}{|c|c|c|c|}
\hline $\begin{array}{l}\text { Decision } \\
\text { Indicator }\end{array}$ & 10 most influencing factors & & 10 most influencing factors \\
\hline \multirow{10}{*}{$\begin{array}{l}\text { Economic } \\
\text { development }\end{array}$} & Employment & \multirow{10}{*}{$\begin{array}{l}\text { Technical } \\
\text { feasibility }\end{array}$} & $\begin{array}{l}\text { Complexity of implementation } \\
\text { method }\end{array}$ \\
\hline & Trade & & Construction phase safety \\
\hline & Local business & & Capital funding \\
\hline & $\begin{array}{l}\text { Incomes of business and property } \\
\text { owners }\end{array}$ & & Project location \\
\hline & Freight & & Construction cost \\
\hline & Inward investment & & Safety improvement \\
\hline & Business productivity & & Construction time period \\
\hline & Tourism & & Resilience to natural hazards \\
\hline & Convention business & & Community health \\
\hline & Making greater labor force available & & Availability of fund \\
\hline \multirow{10}{*}{$\begin{array}{l}\text { Social } \\
\text { development }\end{array}$} & Community health & \multirow{10}{*}{$\begin{array}{l}\text { Project } \\
\text { cost }\end{array}$} & Construction cost \\
\hline & Protect neighborhood characteristics & & Land acquisition cost \\
\hline & Households satisfaction & & Construction time period \\
\hline & Safety improvement & & $\begin{array}{l}\text { Complexity of implementation } \\
\text { method }\end{array}$ \\
\hline & Protect landscape, heritage and history & & Maintenance cost \\
\hline & Relocation of residents or businesses & & Capital funding \\
\hline & Land and habitat protection & & Legal cost \\
\hline & Healthy commute & & Legal issues \\
\hline & $\begin{array}{l}\text { Income of business and property } \\
\text { owners }\end{array}$ & & Resilience to natural hazards \\
\hline & $\begin{array}{l}\text { Offering balanced regional } \\
\text { development }\end{array}$ & & Public-private partnership \\
\hline \multirow{10}{*}{$\begin{array}{l}\text { Protect natural } \\
\text { environment }\end{array}$} & Reduce noise pollution & \multirow{10}{*}{$\begin{array}{l}\text { Financial } \\
\text { feasibility }\end{array}$} & Availability of fund \\
\hline & Land and habitat protection & & Public-private partnership \\
\hline & Maintain air quality & & Legal issues \\
\hline & Global warming & & Capital funding \\
\hline & Water pollution & & Legal cost \\
\hline & Decrease smog and acid rains & & $\begin{array}{l}\text { Complexity of implementation } \\
\text { method }\end{array}$ \\
\hline & Reduce accidents & & Construction cost \\
\hline & Impact of hazardous material & & Construction time period \\
\hline & Reduce fuel consumption & & Resilience to natural hazards \\
\hline & Ecological changes & & Maintenance cost \\
\hline \multirow{10}{*}{$\begin{array}{l}\text { Transportation } \\
\text { benefits }\end{array}$} & Safety improvement & & \\
\hline & Travel cost saving & & \\
\hline & Travel time saving & & \\
\hline & Reduce traffic & & \\
\hline & Reduce fatalities & & \\
\hline & Impact of hazardous material & & \\
\hline & Reduce accidents & & \\
\hline & Global warming & & \\
\hline & Construction phase safety & & \\
\hline & Community health & & \\
\hline
\end{tabular}


Although the ranking of these factors were different for various states of decision indicators, but the differences were negligible. The complete results of sensitivity analysis for "Economic development" decision indicator are presented as an example in the Appendix.

\subsection{Chapter Summary}

This chapter presented the application of the proposed decision support framework to an actual major transportation project in the front-end phases as well as an application of the Bayesian belief model using the real world data from another project. The result of the framework implementation was analyzed and discussed thoroughly. The feedback of the project experts is also explained in this chapter. 


\section{CHAPTER 7. CONCLUSION}

\subsection{Thesis Summary}

Decision-making process of major transportation projects is very challenging for transportation agencies because of their inherent complication. Such projects attract a high level of public attention and political interest not only due to their considerable cost, but principally because of their substantial and long lasting direct and indirect development impacts on communities, environments economies and institutions at local, regional, national and international levels. There is a dynamic relationship between different stakeholders and decision-making parties and the MTP decision-making mechanism is governed by this dynamic relationship.

Major Transportation Projects are recognized as complicated projects with complex decision-making process, especially in the beginning phase of project planning. The inherent uncertainty as well as numerous stakeholders with conflicting interests makes this process more difficult. To address this problem, current research provided a decision framework to optimize the decision-making in front-end phase of MTPs. To achieve this goal, first a comprehensive inventory of factors and decision indicators influencing in decision-making process of MTPs at the front-end phase are identified. Then two cases of MTPs are studied and based on the obtained information the framework has been developed. The framework facilitates quantification of their collective impact on the alternative assessment procedure. The Bayesian belief networkbased analysis can address the identified gaps in the current methods, such as considering

the uncertainty and role of various stakeholders. To illustrate the model, an example 
problem has been solved using the proposed framework and it shows the application of the BNN model and its ability to easily simulate the problem. This framework can be customized for every MTP based on specific needs and conditions.

Considering the context of decision-making in the early stage of large size transportation projects, this dissertation described a decision support tool to evaluate the MTP alternatives and to assist the user in developing a suitable decision-making strategy for a particular project under consideration. The potential influencing factors on the frontend phase of MTPs were identified through a extensive literature review and a questionnaire survey as well as a series of interviews with the US Department of Transportation personnel who had expertise in various aspects of transportation planning,. The published guidelines and regulations regarding the subject matter were also reviewed for this purpose.

A generic Bayesian belief network was established based on the interrelationships between the factors to illustrate the influence of the factors on each other and also on the decision indicators for the project under consideration. Moreover, an overview of the state-of-art of process of the front-end phase of MTPs research was presented and the limitations of the current methods were discussed.

\subsection{Summary of Results}

Chapter 2 investigated the past studies and primary researches about the process of the front-end phase in major transportation projects and provided a detailed review of relevant publications. Chapter 3 presented a review of current practices and regulations in the US transportation agencies 
Chapter 4 discussed the proposed framework step by step in details to assist the readers in understanding the created modules as well as inputs and outputs of each module. The basic theories and mathematic formulations used in the framework were also described in Chapter 4.

A detailed discussion of the factors that have a potential impact on the decisionmaking process of major transportation project in the front-end stage was provided in Chapter 5. This chapter also discussed the development of a generic belief network for MTPs using the matrix approach based on the cause-effect relationships between variables. Different levels of factors and the associated conditional probability table for each factor were also explained in this chapter.

To demonstrate the application of the model, Chapter 6 presented the application of the proposed decision support tool to a completed DRIC project from Michigan Department of Transportation. The operation and function of the three major modules of the methodology introduced in Chapter 4 were described in detail there. Three access alternatives (roadway and railway and marine access) were evaluated using the concepts of conditional probabilities and multi criteria decision-making approach. Based on the model results, the "roadway" alternative should be selected by decision-makers.

\subsection{Research Contributions}

This dissertation contributes to existing literature by demonstrating the possibility of combining a Bayesian belief network and multi criteria decision-making method to enhance the decision-making mechanism in the front-end phase of major transportation projects. The objective of this research was to mitigate the impacts of high uncertainty in 
the front-end phase of MTPs as well as understand and analyze the dynamic relationship between the parties in decision-making process.

The uses of the proposed decision support tool for transportation agencies in this dissertation is threefold: (i) developing planning and decision-making strategy, (ii) identifying the impact of factors influencing the front-end phase performance, (iii) deciding about desirability of each project alternative for different stakeholders based on their specific preferences. Similar benefits are commonly acquired in by synthesizing all the information using agencies' historic data, or in users mind. This promotes the transparency, explicitness and robustness of decision-making process. The created method throughout this dissertation establishes the state probabilities of the collected list of influential factors in the front-end phase of MTPs. The aggregated impacts of the considered factors on various decision indicators are also calculated by the model.

The described method in this dissertation assists the user in selecting the optimal alternative among different project alternatives based on the probabilities of different states of decision indicators and desirability of those alternatives. Specially, the main contributions of this research to the existent body of knowledge are:

1. A framework that shows the dynamic interrelationships between the factors impacting the front-end phase of major transportation projects at a macro level, and formulation and quantification of these interrelationships to facilitate prediction of decision indicators with respect to different alternative scenarios considered. 
2. A method to facilitate the use of the stochastically quantified indicators mentioned above for comparing alternative project strategies taking into account the inherent variability in construction operations.

3. A multi criteria decision-making method to consider the preferences of various decision-makers and importance of their role in the decision-making mechanism.

The decision support tool presented in this research provides the user with four main outputs with respect to a new project:

1. The state probabilities of factors impacting the project decision indicators.

2. The state probabilities of project decision indicators reflecting the cumulative impact of factors impacting the project performance,

3. An indicator probability matrix for qualitative alternatives to facilitate a relative comparison,

4. A ranking of qualitative alternatives

One of the advantages of the decision support tool presented in this dissertation is that if the user thinks that a factor does not have significant on that particular project under consideration, it can be simply removed from the belief network or its weight can be adjusted in assigning probabilities accordingly. The model enables to cut-off any unnecessary link between the nodes in the belief network to imply the irrelevance of that particular cause-effect relationship for the project under consideration without impacting other links. Therefore, the methodology described in this research is applicable to other type and size of projects and the state Departments of Transportation can use it for a wide range of different projects. 


\subsection{Limitations and Future Research Potential}

The created decision support tool in this dissertation is only proposed to be applied to a roadway, railway or marine link between two sides of the river due to the type and level of detail of factors included in the framework. Future research could include other types of major transportation projects or other infrastructure projects.

The developed model only allows input from a single user. A group decision module, with respect to the conditional probabilities of factor relationships and the relative weights between different project variables, could be integrated into the developed model to increase the efficiency and accuracy of the input and therefore the results.

Finally, the methodology developed in this dissertation can be applied to projects other than transportation infrastructure. With some modification, the model can be used with the same purposes for analysis of the construction of new facilities. Research could be directed towards developing a decision support system to include environmental impact assessment, financial feasibility, and project life cycle cost considerations of water infrastructure projects. 


\section{REFERENCES}

Ambrasaite, I., Barfod, M. B., and Salling, K. B. (2011). "MCDA and risk analysis in transport infrastructure appraisals: The rail baltica case." Procedia-Social and Behavioral Sciences, 20 944-953.

Attoh-Okine, N. O., and Gibbons, J. (2001). "Use of belief function in brownfield infrastructure redevelopment decision making." J.Urban Plann.Dev., 127(3), 126-143.

Barfod, M. B., Salling, K. B., and Leleur, S. (2011). "Composite decision support by combining cost-benefit and multi-criteria decision analysis." Decis.Support Syst., 51(1), 167-175.

Bayraktar, M. E., and Hastak, M. (2009). "A decision support system for selecting the optimal contracting strategy in highway work zone projects." Autom.Constr., 18(6), 834843.

Berechman, J., and Paaswell, R. E. (2005). "Evaluation, prioritization and selection of transportation investment projects in New York City." Transportation, 32(3), 223-249.

Beukers, E., Bertolini, L., and Te Brömmelstroet, M. (2012). "Why Cost Benefit Analysis is perceived as a problematic tool for assessment of transport plans: A process perspective." Transportation Research Part A: Policy and Practice, 46(1), 68-78.

Bruzelius, N., Flyvbjerg, B., and Rothengatter, W. (2002). "Big decisions, big risks. Improving accountability in mega projects." Transp.Policy, 9(2), 143-154.

CII. (2012). "Adding Value through Front End Planning." Rep. No. CII Special

Publication 268-3, Construction Industry Institute, University of Texas at Austin.

Dey, P. K. (2006). "Integrated project evaluation and selection using multiple-attribute decision-making technique." Int J Prod Econ, 103(1), 90-103.

FHWA Office of Innovative Program Delivery, Project Delivery. (2016). "Project Delivery Defined- Major Projects." http://www.fhwa.dot.gov/ipd/project_delivery/defined/major_project.aspx .

FHWA, Transport, C., MDOT, and OMT. (2004). "Canada-U.S.-Ontario-Michigan Transportation Partnership Planning/Need and Feasibility Study: Feasible Transportation Alternatives Working Paper." .

Gibson Jr, G. E., Wang, Y., Cho, C., and Pappas, M. P. (2006). "What is preproject planning, anyway?" J.Manage.Eng., 22(1), 35-42. 
Gibson Jr, G., Kaczmarowski, J., and Lore Jr, H. (1995). "Preproject-planning process for capital facilities." J.Constr.Eng.Manage., 121(3), 312-318.

Giezen, M., Bertolini, L., and Salet, W. (2014). "Adaptive Capacity Within a Mega Project: A Case Study on Planning and Decision-Making in the Face of Complexity." European Planning Studies, (ahead-of-print), 1-20.

Gil, N., and Beckman, S. (2009). "Infrastructure meets business: building new bridges, mending old ones." Calif.Manage.Rev., 51(2), 6-29.

Hall, J. W., Henriques, J. J., Hickford, A. J., Nicholls, R. J., Baruah, P., Birkin, M., Chaudry, M., Curtis, T. P., Eyre, N., and Jones, C. (2014). "Assessing the Long-Term Performance of Cross-Sectoral Strategies for National Infrastructure." J Infrastruct Syst, .

Hall, R. P. (2006). Understanding and Applying the Concept of Sustainable Development to Transportation Planning and Decision-Making in the US, .

Jia, G., Yang, F., Wang, G., Hong, B., and You, R. (2011). "A study of mega project from a perspective of social conflict theory." Int.J.Project Manage., 29(7), 817-827.

Kjaerulff, U. B., and Madsen, A. L. (2008). "Bayesian networks and influence diagrams." Springer Science Business Media, 200114.

Kwak, Y. H., Chih, Y., and Ibbs, C. W. (2009). "Towards a comprehensive understanding of public private partnerships for infrastructure development." Calif.Manage.Rev., 51(2), 51-78.

Lee, D. (2000). "Methods for evaluation of transportation projects in the USA." Transp.Policy, 7(1), 41-50.

Lee, E., Park, Y., and Shin, J. G. (2009). "Large engineering project risk management using a Bayesian belief network." Expert Syst.Appl., 36(3), 5880-5887.

Li, H., and Guo, H. L. (2011). "International Journal of Project Management special issue on "Complexities in managing mega construction projects"." Int.J.Project Manage., 29(7), 795-796.

Morisugi, H. (2000). "Evaluation methodologies of transportation projects in Japan." Transp.Policy, 7(1), 35-40.

OECD. (2007). " Infrastructure to 2030. Mapping Policy for Electricity, Water and Transport. Vol. 2." Organization for Economic Co-operation and Development, . 
Priemus, H. (2010a). "Decision-making on mega-projects: drifting on political discontinuity and market dynamics." Ejtir, 10(1), 19-29.

Priemus, H. (2010b). "Mega-projects: Dealing with pitfalls." European Planning Studies, 18(7), 1023-1039.

Saaty, T. L. (1990). "How to make a decision: the analytic hierarchy process." Eur.J.Oper.Res., 48(1), 9-26.

Salling, K. B. (2013). "A New Approach to Feasibility Risk Assessment within Transport Infrastructure Appraisal." Procedia-Social and Behavioral Sciences, 74 468-477.

Salling, K. B., and Banister, D. (2009). "Assessment of large transport infrastructure projects: the CBA-DK model." Transportation Research Part A: Policy and Practice, 43(9), 800-813.

Samset, K. (2008). "How to overcome major weaknesses in mega-projects: the Norwegian approach." Decision-Making on Mega-Projects: Cost-Benefit Analysis, Planning and Innovation, 173-188.

Sinha, K. C., and Labi, S. (2011). Transportation decision making: Principles of project evaluation and programming. John Wiley \& Sons, .

Su, C., Cheng, M., and Lin, F. (2006). "Simulation-enhanced approach for ranking major transport projects." Journal of Civil Engineering and Management, 12(4), 285-291.

Sun, J., and Zhang, P. (2011). "Owner organization design for mega industrial construction projects." Int.J.Project Manage., 29(7), 828-833.

Szyliowicz, J. S. (2003). "Decision-making, intermodal transportation, and sustainable mobility: towards a new paradigm." International Social Science Journal, 55(176), 185 197.

Trucco, P., Cagno, E., Ruggeri, F., and Grande, O. (2008). "A Bayesian Belief Network modelling of organisational factors in risk analysis: A case study in maritime transportation." Reliab.Eng.Syst.Saf., 93(6), 845-856.

F. USDOT. (2015). "A Guide to Transportation Decisionmaking." https://www.planning.dot.gov/documents/GuidetoTransportationDecisionmaking.pdf .

Vickerman, R. (2000). "Evaluation methodologies for transport projects in the United Kingdom." Transp.Policy, 7(1), 7-16. 
Vreeker, R., Nijkamp, P., and Ter Welle, C. (2002). "A multicriteria decision support methodology for evaluating airport expansion plans." Transportation Research Part D: Transport and Environment, 7(1), 27-47.

Williams, T., and Samset, K. (2010). "Issues in front-end decision making on projects." Proj.Manage.J., 41(2), 38-49.

Yun, S., Suk, S., Dai, J., and Mulva, S. P. (2012). "Quantification of Front End Planning Input Parameters in Capital Projects." Bridges, 10 9780784412329.251.

Zak, J. (2010). "Decision support systems in transportation." Handbook on Decision Making, Springer, 249-294. 
APPENDIX 


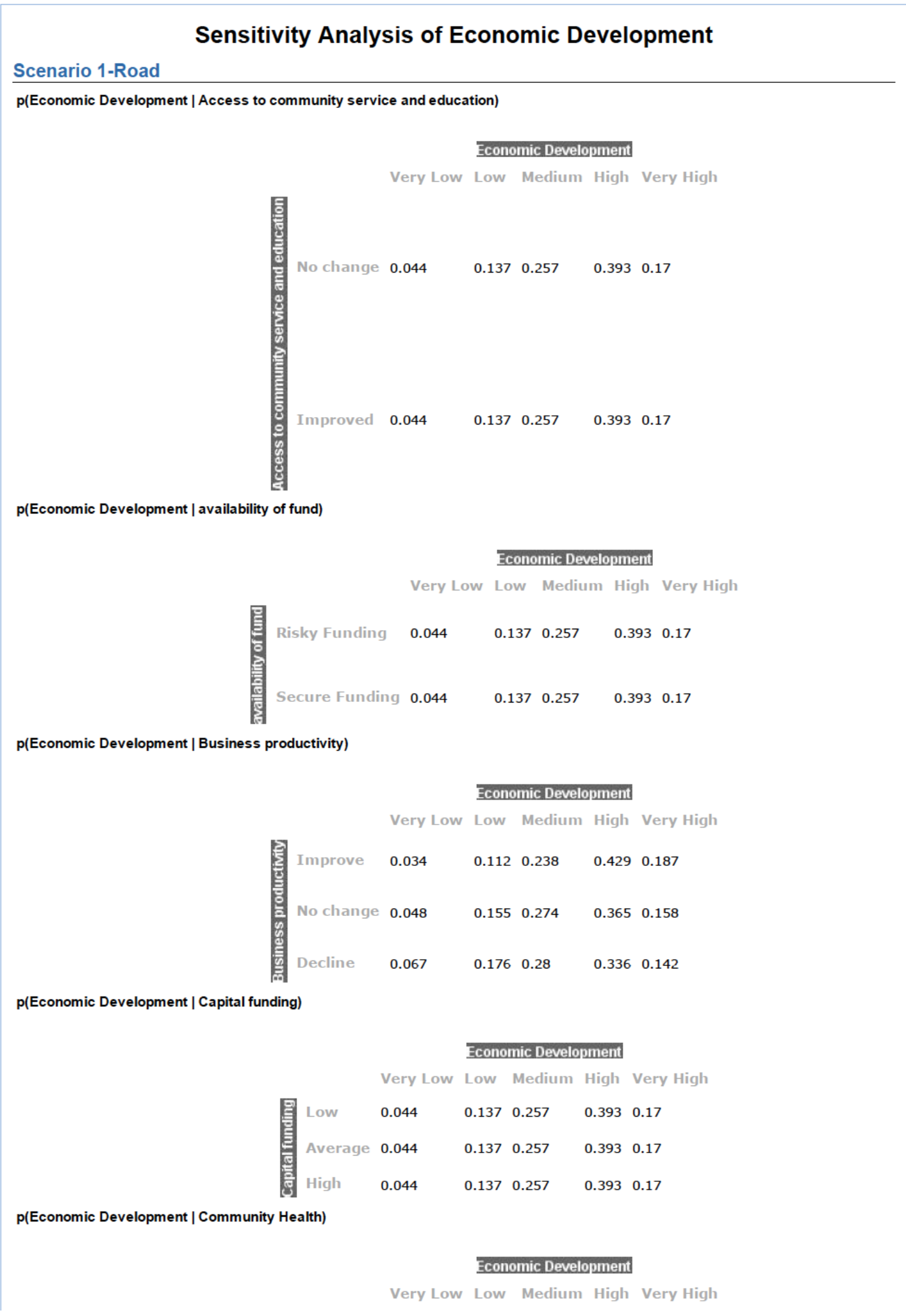




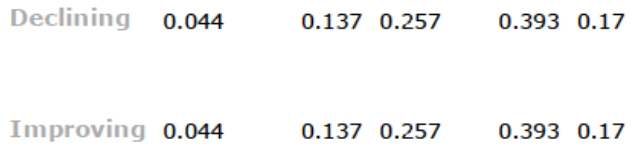

p(Economic Development | Connect various industries and communities)

Very Low Low Medium High Very High

S

Ineffectively 0.061

$0.18 \quad 0.265$

0.3370 .157

Effectively $\quad 0.043$

0.1340 .256

$0.396 \quad 0.171$

p(Economic Development | Construction phase safety)

\section{Economic Development}

Very Low Low Medium High Very High

쥰

$\begin{array}{llllll}\text { Low } & 0.044 & 0.137 & 0.257 & 0.393 & 0.17\end{array}$

$\begin{array}{llllll}\text { High } & 0.044 & 0.137 & 0.257 & 0.393 & 0.17\end{array}$

p(Economic Development | Construction time period) 


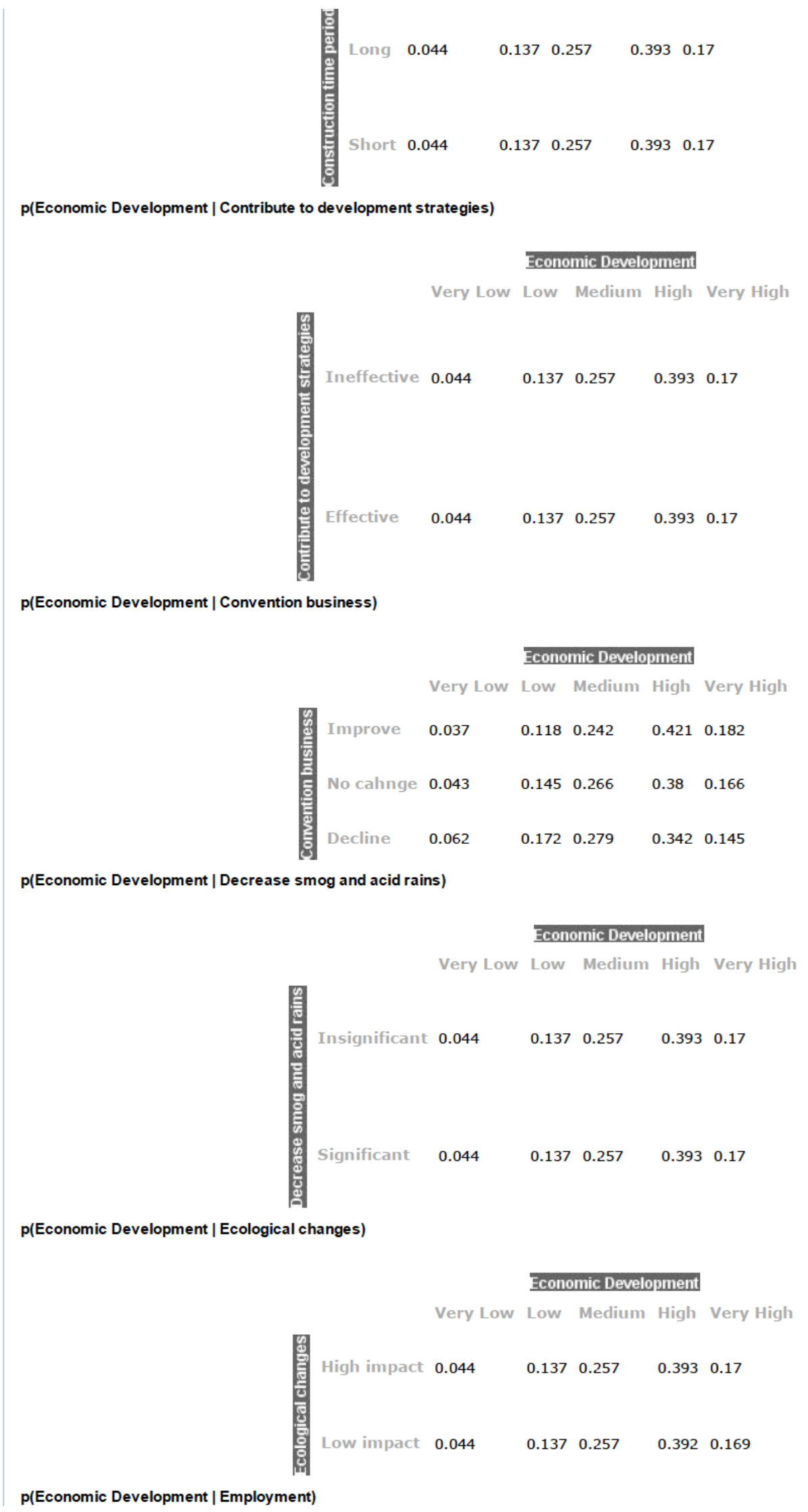




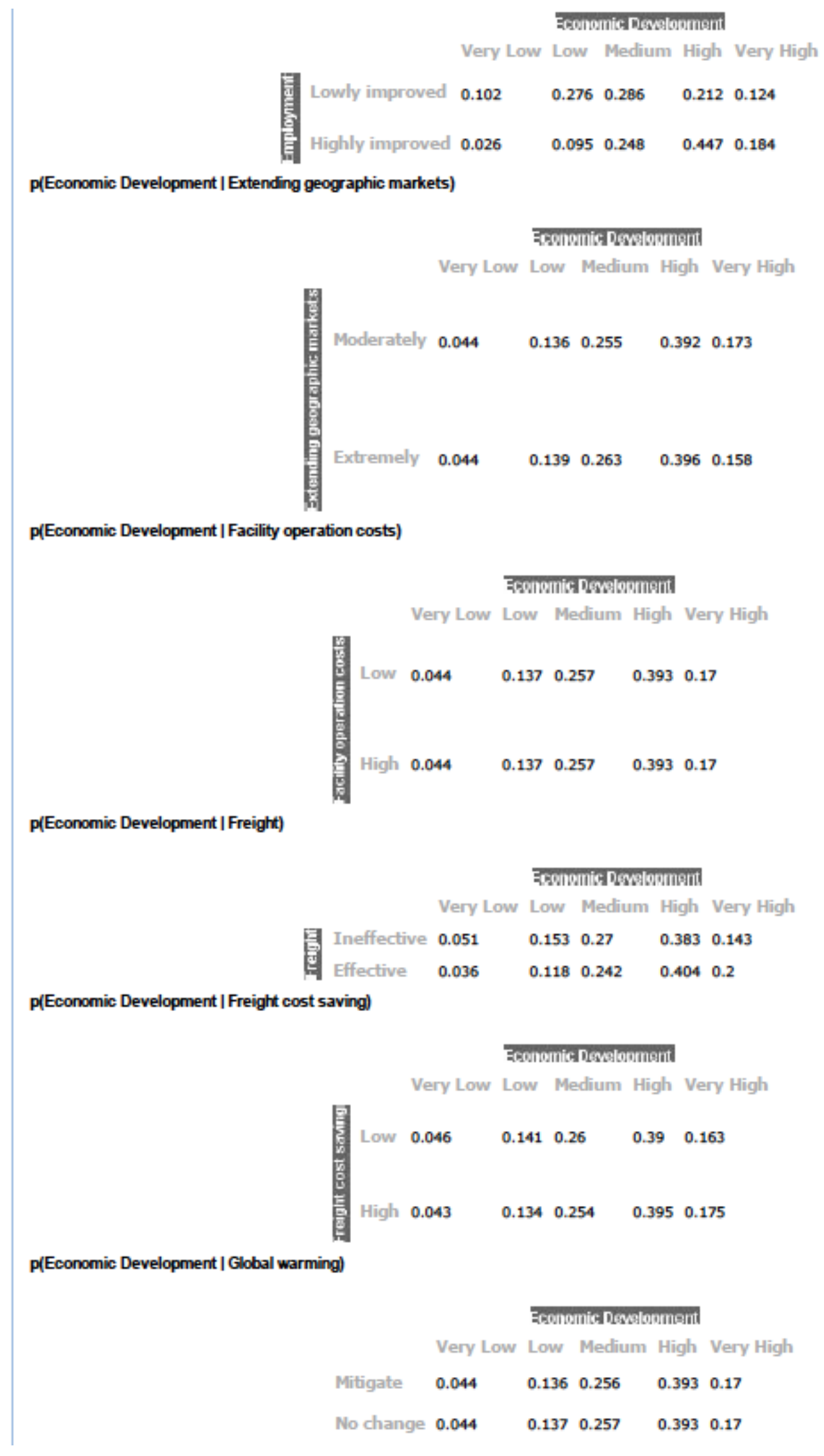




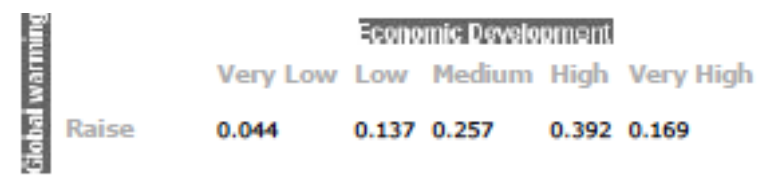

p(Economic Development | Healthy commute)

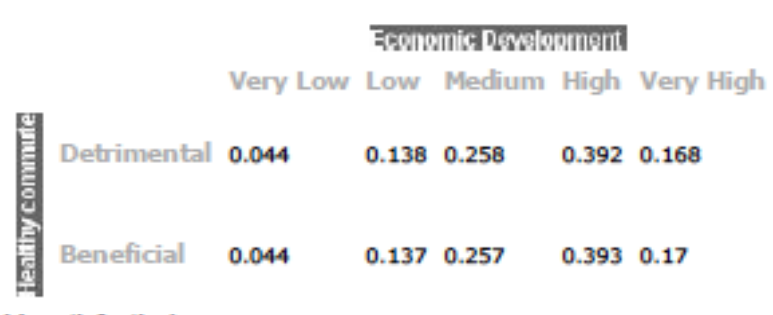

p(Economic Development | Households satisfaction)

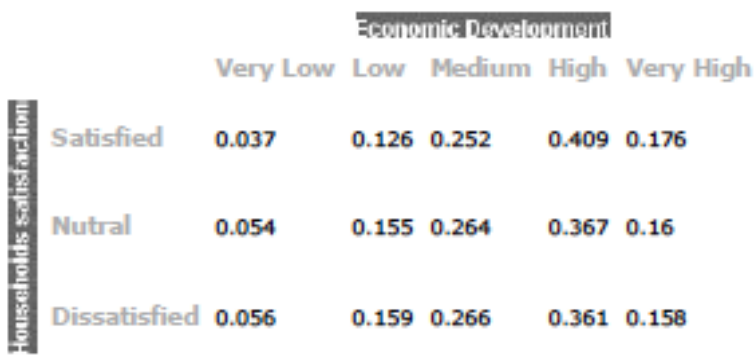

p(Economic Development | Impact of hazardous material)

Economic Dowsopment

Very Low Low Medium High Very High

产

\begin{tabular}{llllll}
\hline Low & 0.044 & 0.138 & 0.258 & 0.392 & 0.168
\end{tabular}

靠

送

$\begin{array}{llllll}\text { High } 0.044 & 0.136 & 0.256 & 0.393 & 0.17\end{array}$

奠

p(Economic Development | Incomes of business and property owners)

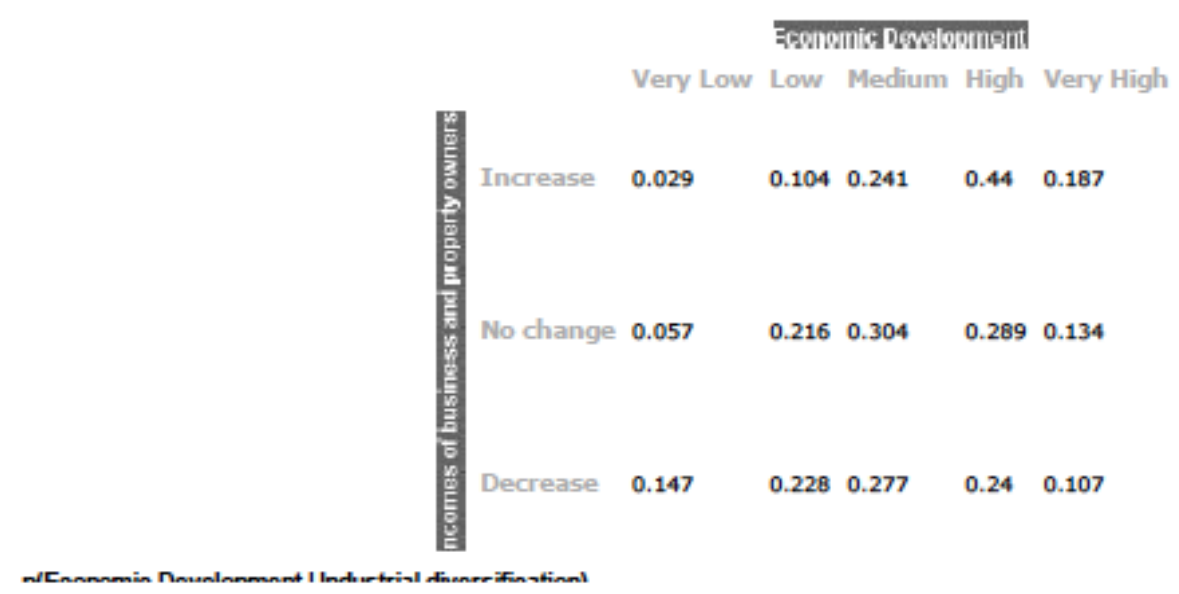




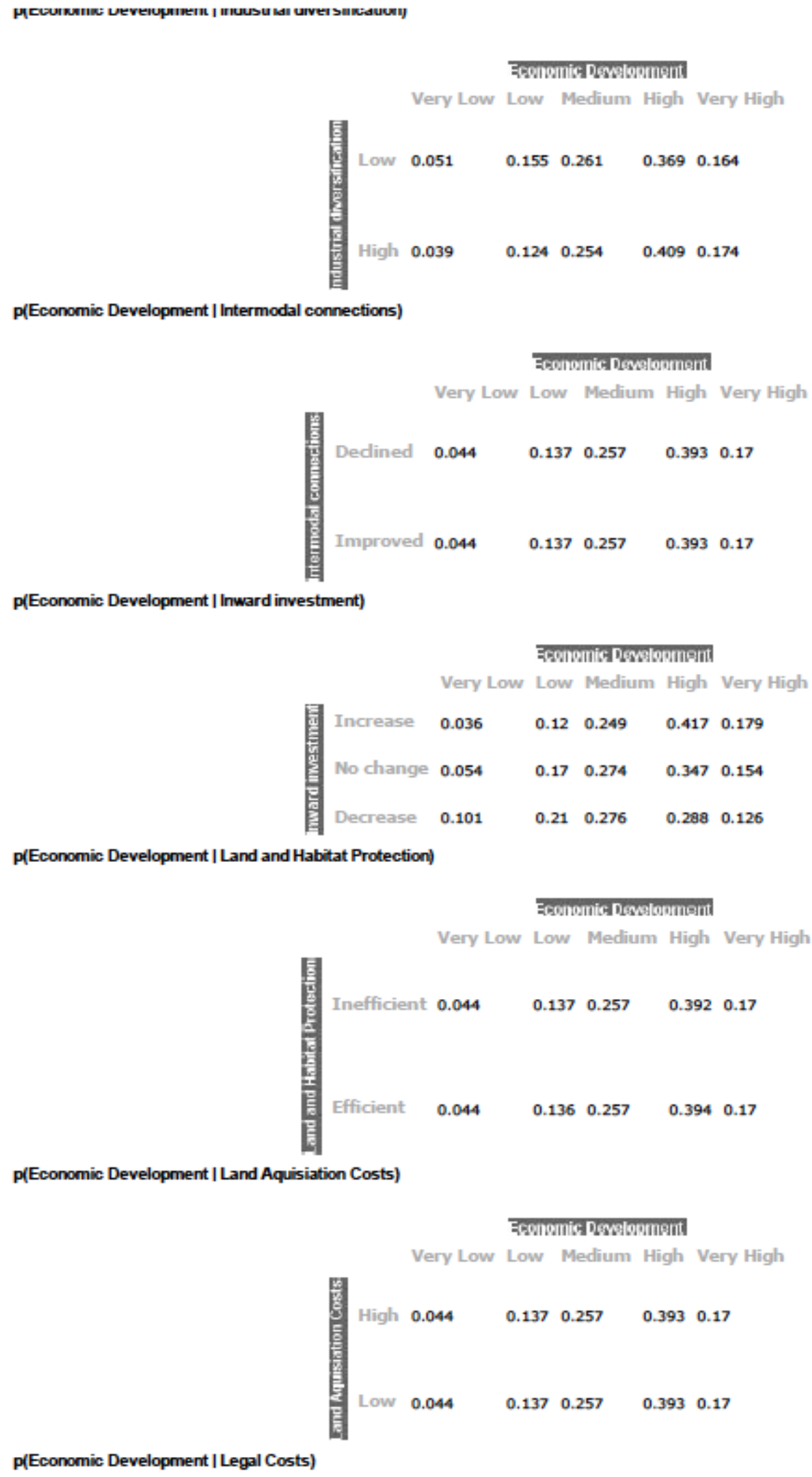

p(Economic Development | Land and Habitat Protection)

p(Economic Development / Land Aquisiation Costs)

Economic Dowopmant

Very Low Low Medium High Very High

E

$\begin{array}{llllll}\text { High } & 0.044 & 0.137 & 0.257 & 0.393 & 0.17\end{array}$

$\begin{array}{llllll}\text { Low } & 0.044 & 0.137 & 0.257 & 0.393 & 0.17\end{array}$

p(Economic Development I Legal Costs) 


\section{Economic Desstosment}

Very Low Low Medium High Very High

$\begin{array}{lllllll}\text { High } & 0.044 & 0.137 & 0.257 & 0.393 & 0.17 \\ \text { Low } & 0.044 & 0.137 & 0.257 & 0.393 & 0.17\end{array}$

p(Economic Development | Legal issues)

Economichesolonment

Very Low Low Medium High Very High

\begin{tabular}{lllllll}
\hline Major & 0.044 & 0.137 & 0.257 & 0.393 & 0.17 \\
Common & 0.044 & 0.137 & 0.257 & 0.393 & 0.17
\end{tabular}

p(Economic Development | Local Business)

Econounich Dewolosment

Very Low Low Medium High Very High

$\begin{array}{llllllll}\text { Improve } & 0.025 & 0.087 & 0.22 & 0.465 & 0.202 \\ \text { No change } & 0.04 & 0.189 & 0.314 & 0.314 & 0.143 \\ \text { Decline } & 0.144 & 0.241 & 0.282 & 0.249 & 0.084\end{array}$

p(Economic Development | Maintain Air Quality)

\section{Zconomic Dsovopment}

Very Low Low Medium High Very High

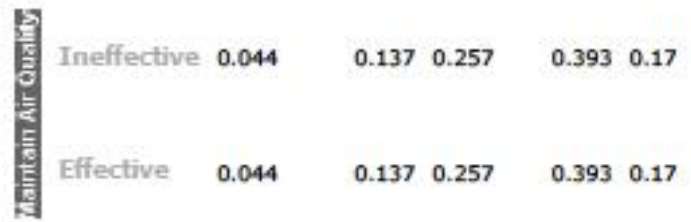

p(Economic Development | Maintenance Costs)

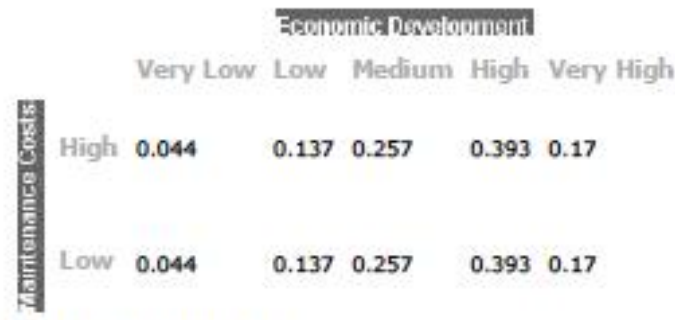

p(Economic Development | Making a greater labor force avallable)

$$
\text { 5conomic Dswelopment }
$$

Very Low Low Medium High Very High

$$
\begin{array}{llllll}
\text { Low } 0.061 & 0.183 & 0.266 & 0.333 & 0.157
\end{array}
$$




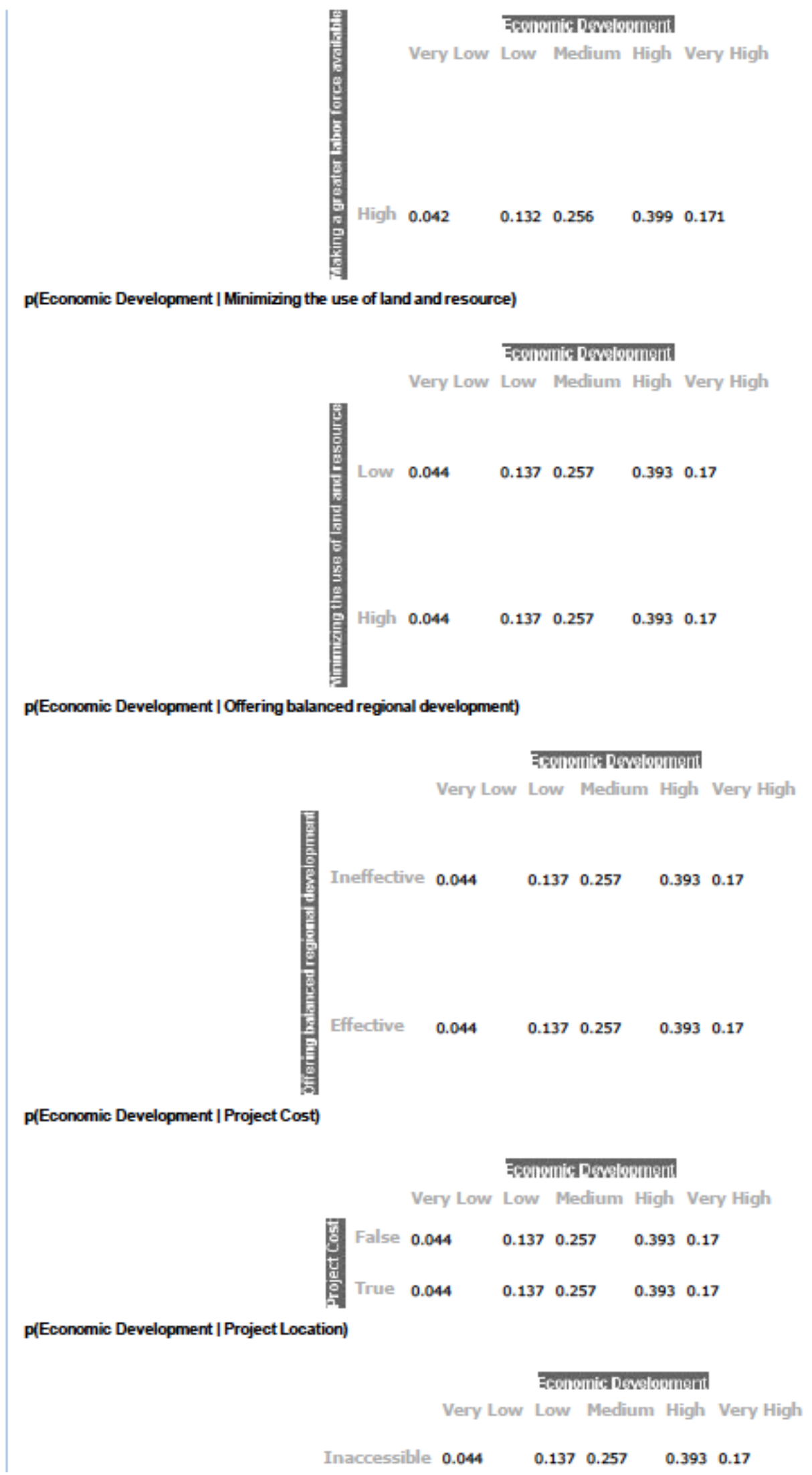




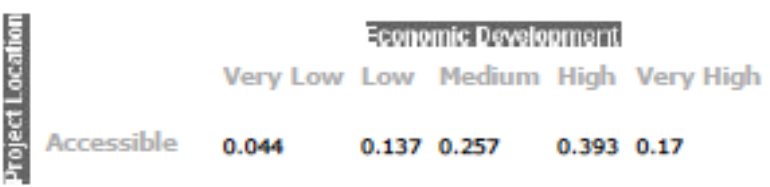

p(Economic Development | Protecting landscape, heritage, history)

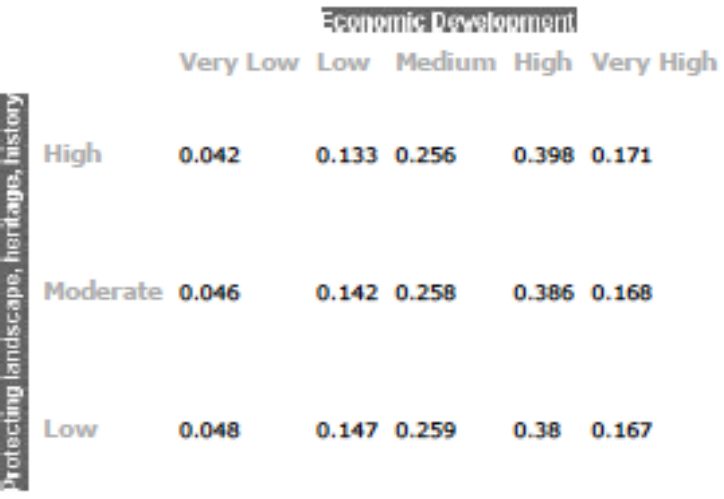

p(Economic Development | Protect neighborhood characteristics)

Economic Desolopmant

Very Low Low Medium High Very High

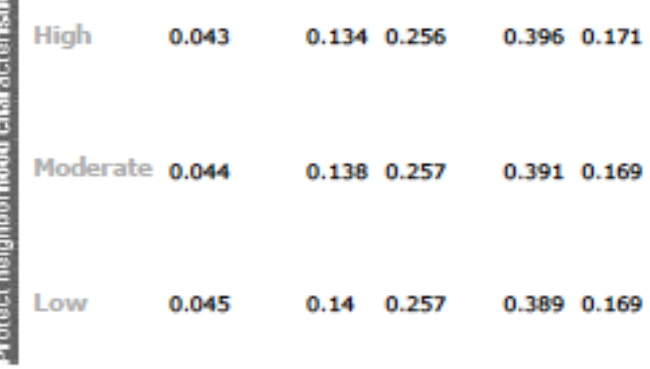

p(Economic Development | Public_Private Partnership)

Economic Dowlonmant

Very Low Low Medium High Very High

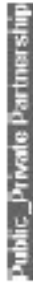

Impossible $0.044 \quad 0.137 \quad 0.257 \quad 0.393 \quad 0.17$

$\begin{array}{llllll}\text { Possible } & 0.044 & 0.137 & 0.257 & 0.393 & 0.17\end{array}$

p(Economic Development | Quality of trips)

Economic Dowopment

Very Low Low Medium High Very High

\begin{tabular}{lllllll}
\hline Stressful & 0.046 & 0.141 & 0.259 & 0.389 & 0.165 \\
& & & & & & \\
Unstressed & 0.042 & 0.133 & 0.255 & 0.396 & 0.174
\end{tabular}

p(Economic Development | Reduce accidents) 
Economic Dowstonment

Very Low Low Medium High Very High

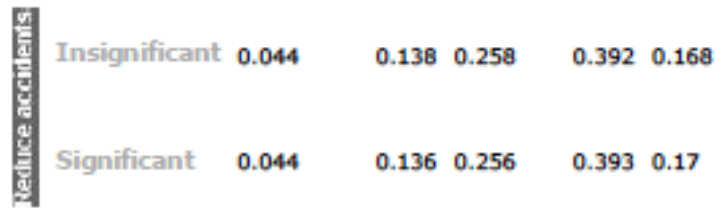

p(Economic Development | Reduce fatalities)

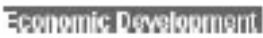

Very Low Low Medium High Very High

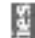

$\begin{array}{llllll}\text { Low } & 0.044 & 0.137 & 0.257 & 0.392 & 0.169\end{array}$

赵

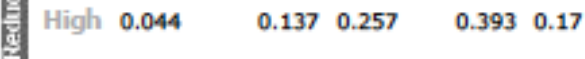

p(Economic Development | Reduce fule consumption)

\section{Economic Dewelopment}

Very Low Low Medium High Very High

E

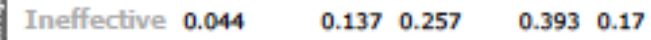

$\begin{array}{llllll}\text { Effective } & 0.044 & 0.137 & 0.257 & 0.393 & 0.17\end{array}$

p(Economic Development | Reduce noise pollution)

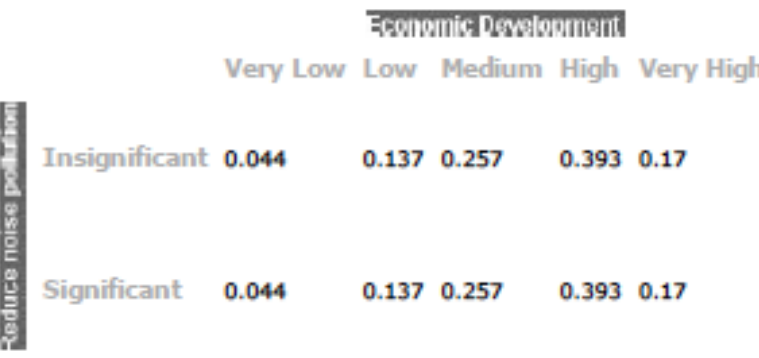

p(Economic Development | Reduce traffic)

Economice Dowopmant

Very Low Low Medium High Very High

\begin{tabular}{lllllll}
\hline 最 & Low & 0.044 & 0.138 & 0.257 & 0.392 & 0.169
\end{tabular}

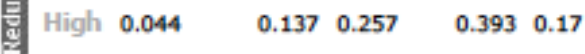

p(Economic Development | Reliability of travel mode) 

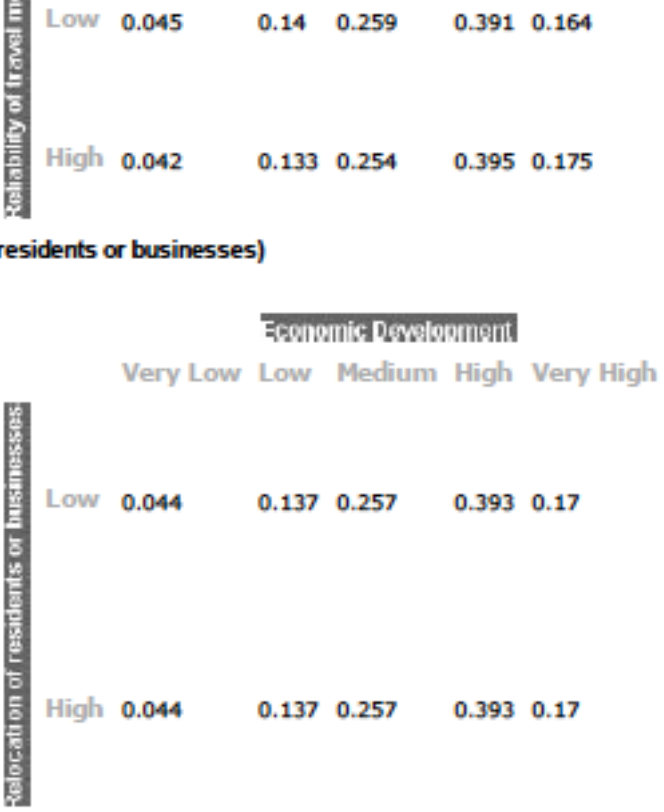

p(Economic Development | Resilience to natural hazards)

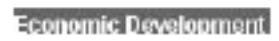

Very Low Low Medium High Very High

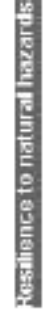

$\begin{array}{llll}0.137 & 0.257 & 0.393 & 0.17\end{array}$

$\begin{array}{lllll}\text { High } 0.044 & 0.137 & 0.257 & 0.393 & 0.17\end{array}$

p(Economic Development | Safety improvement)

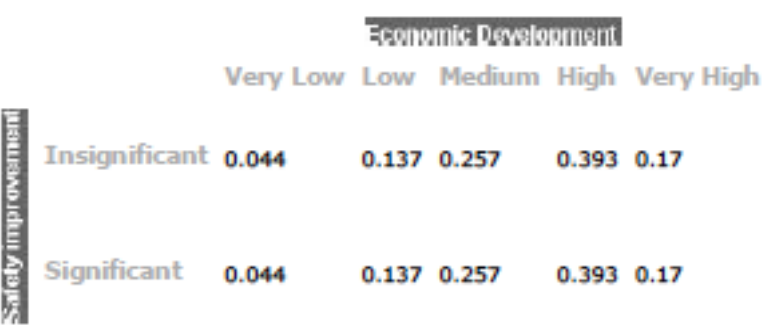

p(Economic Development | Tourism)

Economic Dosolopment

Very Low Low Medium High Very High

言

0.036

$0.117 \quad 0.253$

0.4190 .176

No change $\mathbf{0 . 0 4 7}$

$\begin{array}{lll}0.146 & 0.259\end{array}$

0.3820 .167

Decline $\quad 0.054$

$0.162 \quad 0.262$

$0.36 \quad 0.162$

p(Economic Development I Trade)

Econvmic Desolopment 


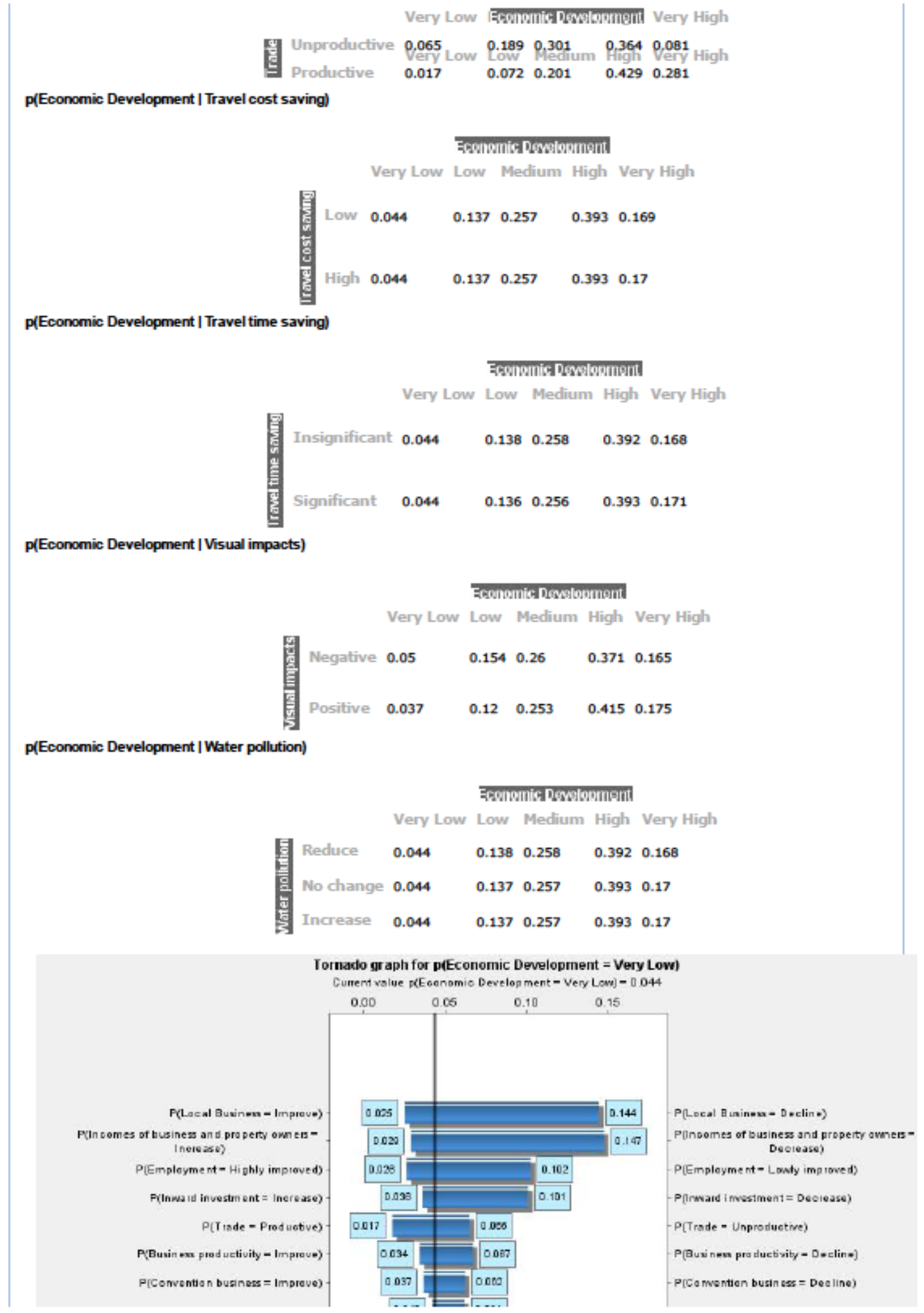




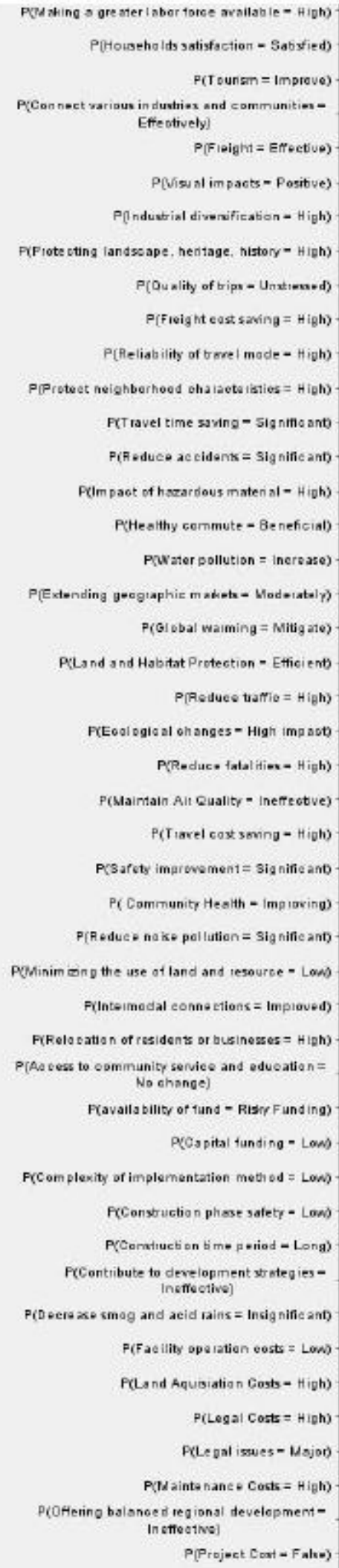

PQd zhing s are ster 1 aber toree avallable - High)

P(Househalds satisfaction - Sabsied)

$\mathrm{P}$ (Tounsan $=$ Impreve $)$

PXQConnect garious in dustriox and communitien Effeotively]

$P(F \mid$ eight $=$ Enfoetive $)$

$P($ visu al impacts - Positive $)$

P(gndustrial diverification - High)

P(Fiote cting landso ape, heriage, history- High)

$P($ Quality of tripu - Unztened)

P(Fivight eost saving $=H$ igh $)$

P(teliability of torrel made - High)

P(Protect neighberhoed oha laets isties $=$ Hidh)

F(T ravel time saving- signmo ant

$P(F=d u c e$ ac cidens = signific ant

F(Im paot of hazardous materis - Hioh)

P(Hesthy cormmute - 8e neficial)

PRWater pollution $=$ incroase)

$P($ Extending gecgiaphie makets - Modetatoly)

$P($ Global waiming $=$ Mitig ate $)$

PLand and Habiat Protection - Etticients

P(Reduee taffile $=\mathrm{High})$

P(Eool ogio al oh anges - High impaot)

PReduc= fatal itiex - High)

P(Maintain Alt Quality - Ineffscotve)

$P(T$ iavel cost sonng - Hiph $)$

$P($ Safoty imarowement $=$ signific ant

P( Community Hesith - Imp Iseing)

$P(R$ \&due o nake pol lution = signifie ant $)$

POGhim on o the use of land and resource - Lans

F(Inteimedal conne ctions = Impioved $)$

F(Relosation of residents or businesses $=H i g h)$

PiAd cess to eommunity service and edueation $=$ No ehange)

F(avalla bility of tund - Risir F und ing)

Prospital fundino - Lams

F(Complexity of implementzation metiod $=$ Low)

F(Construction phose satety - Lons)

P(Comatuction time period - Long)

FCContribute to development strotegies Instrective]

$P(D$ eers $x$ so smog and aeid rains = Insignine ant $)$

$F(F a c$ ility operatien east $=$ Low $)$

P(Land Aquisiation Gosts - Hiph)

$P($ Legal Coats $=H i g h)$

PQLe gal iswes - Mojor)

P(d ainto hane $=$ Costs $=\mathrm{High})$

ProHerino balonoed regional development -

In etteative]

P(Project Coas - Fales) 


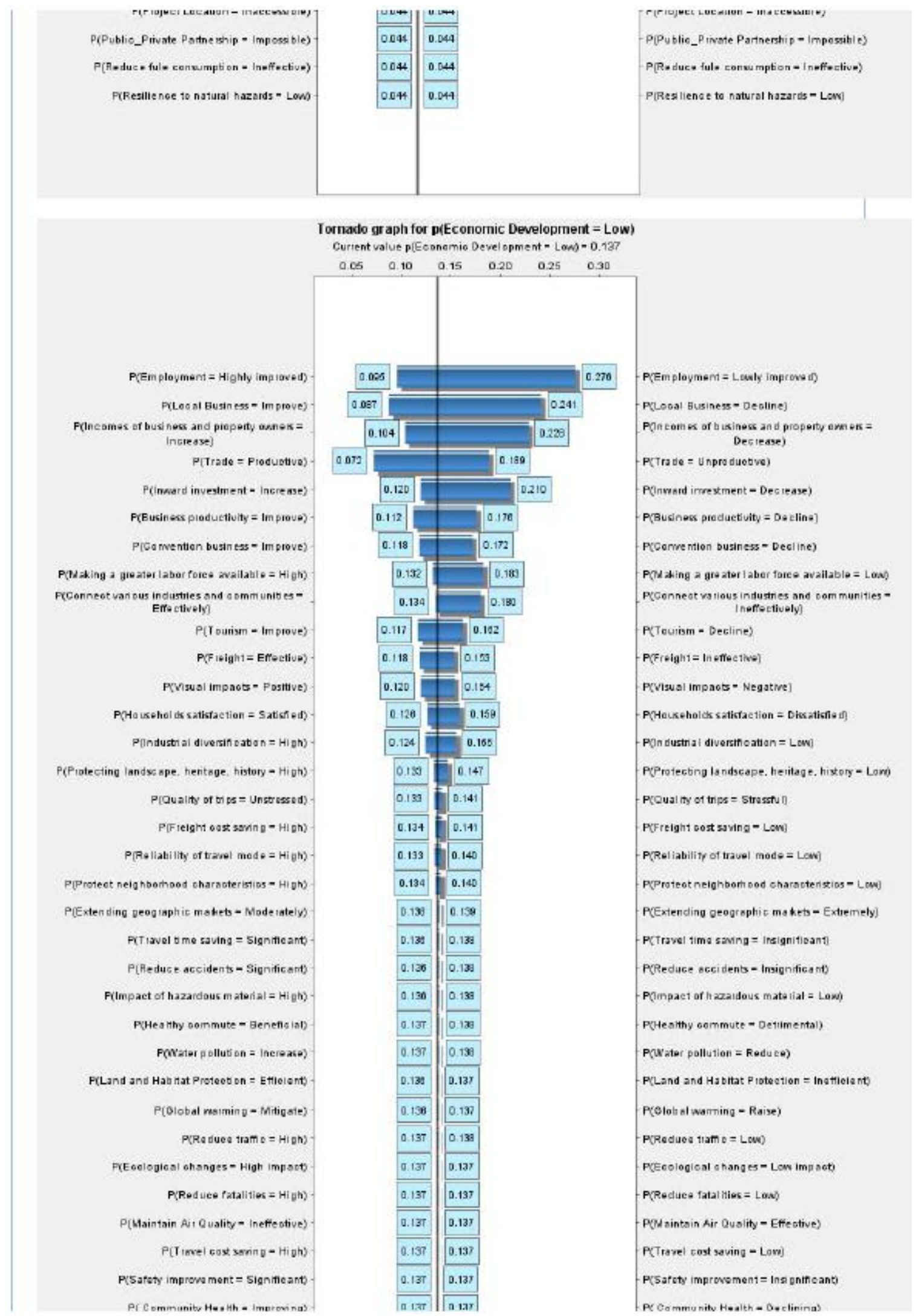




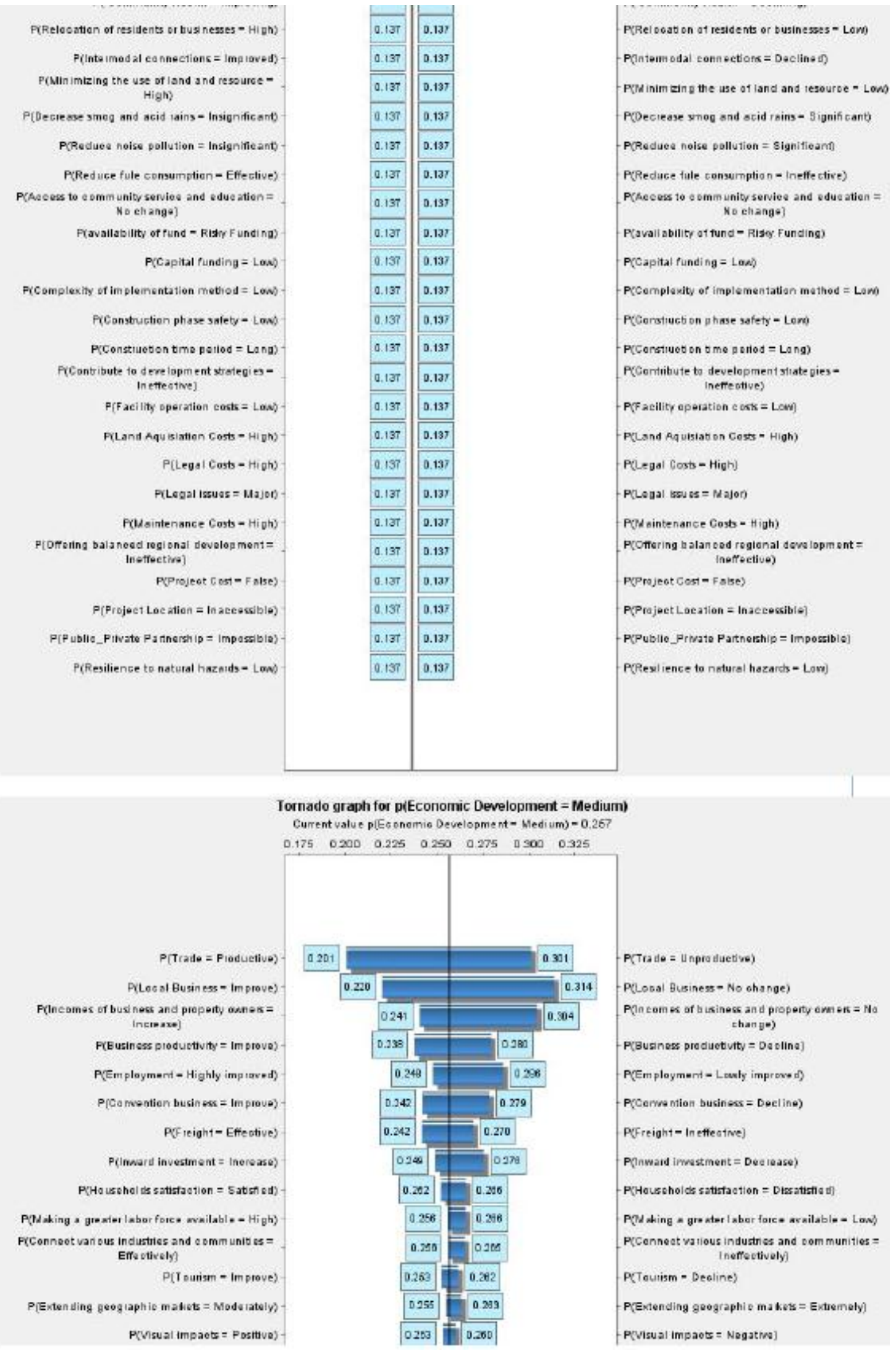



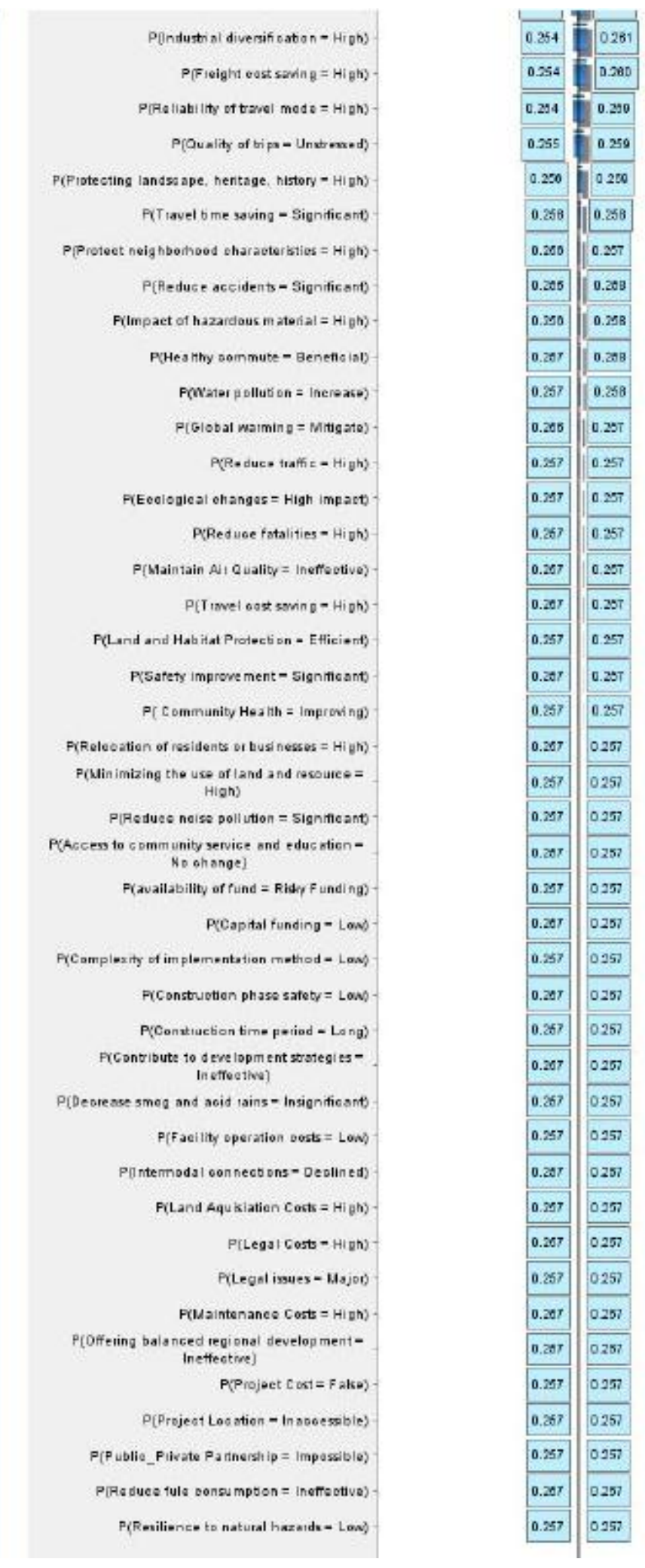

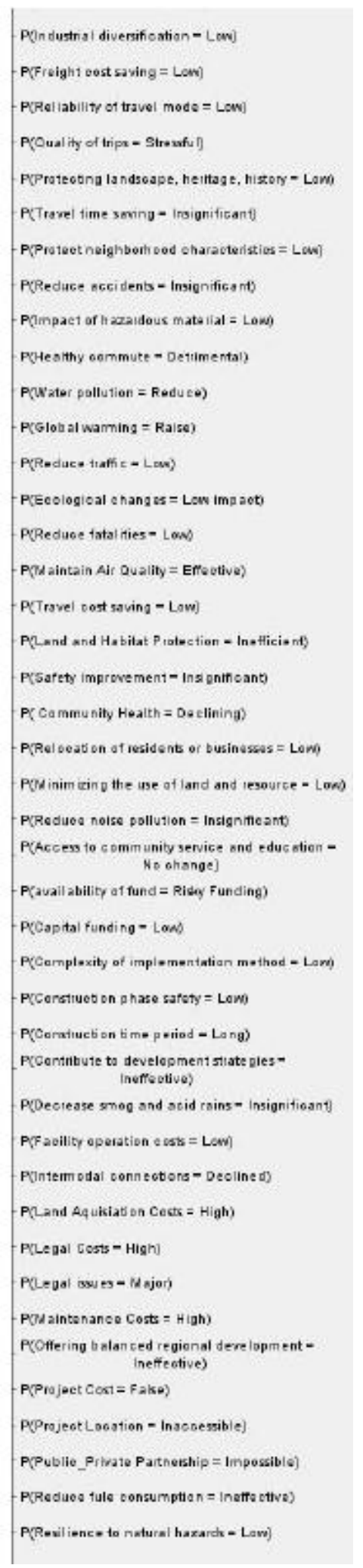




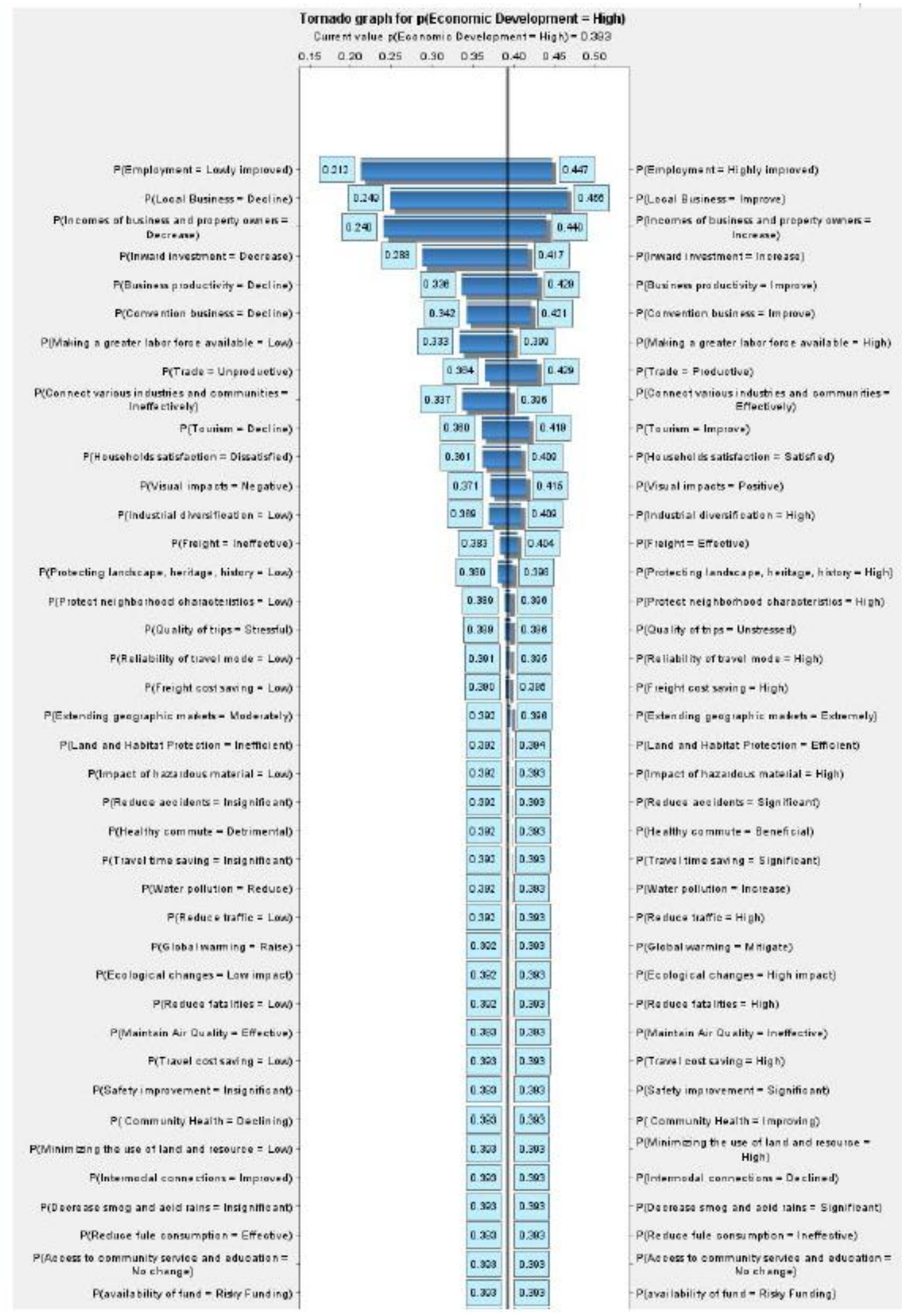




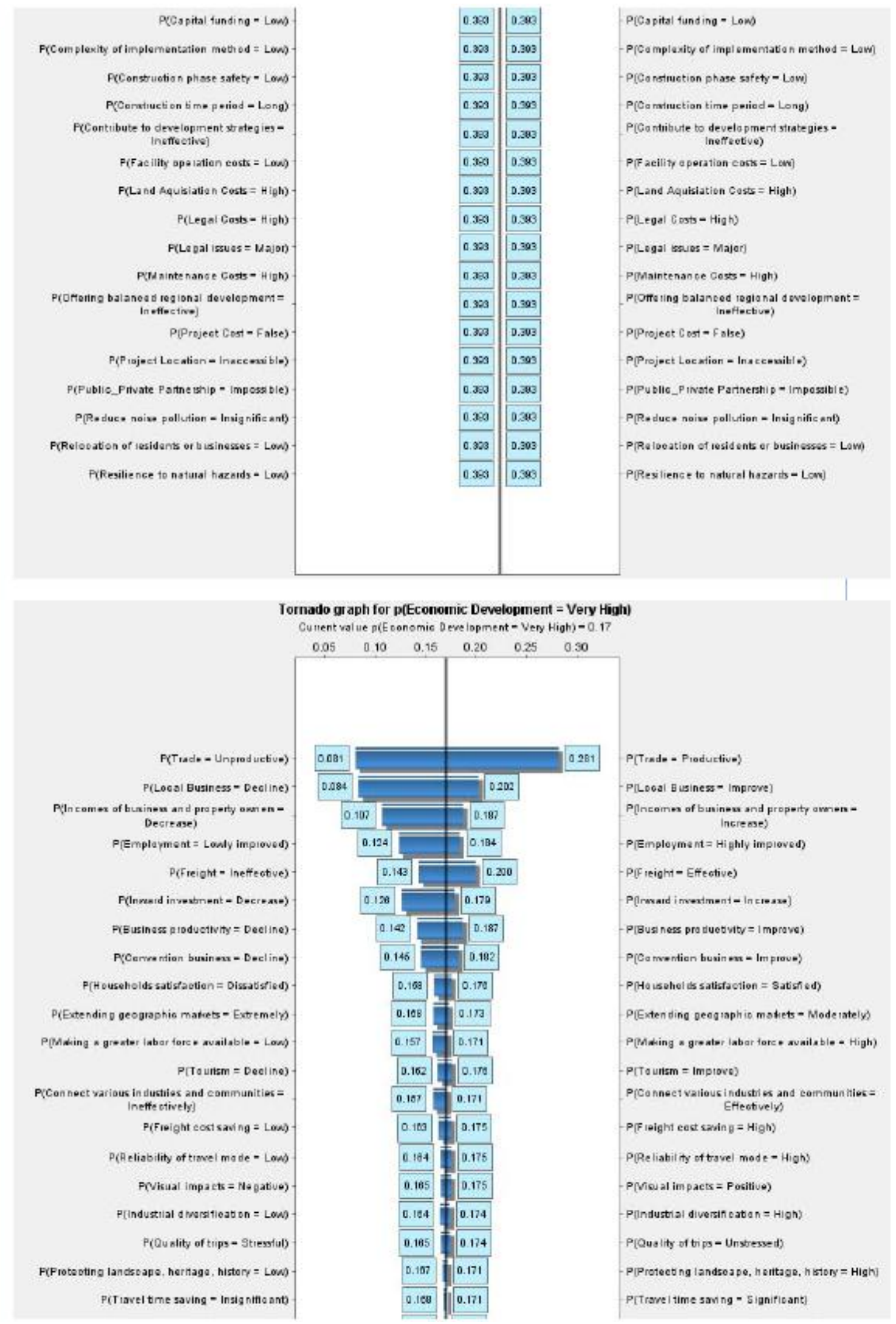




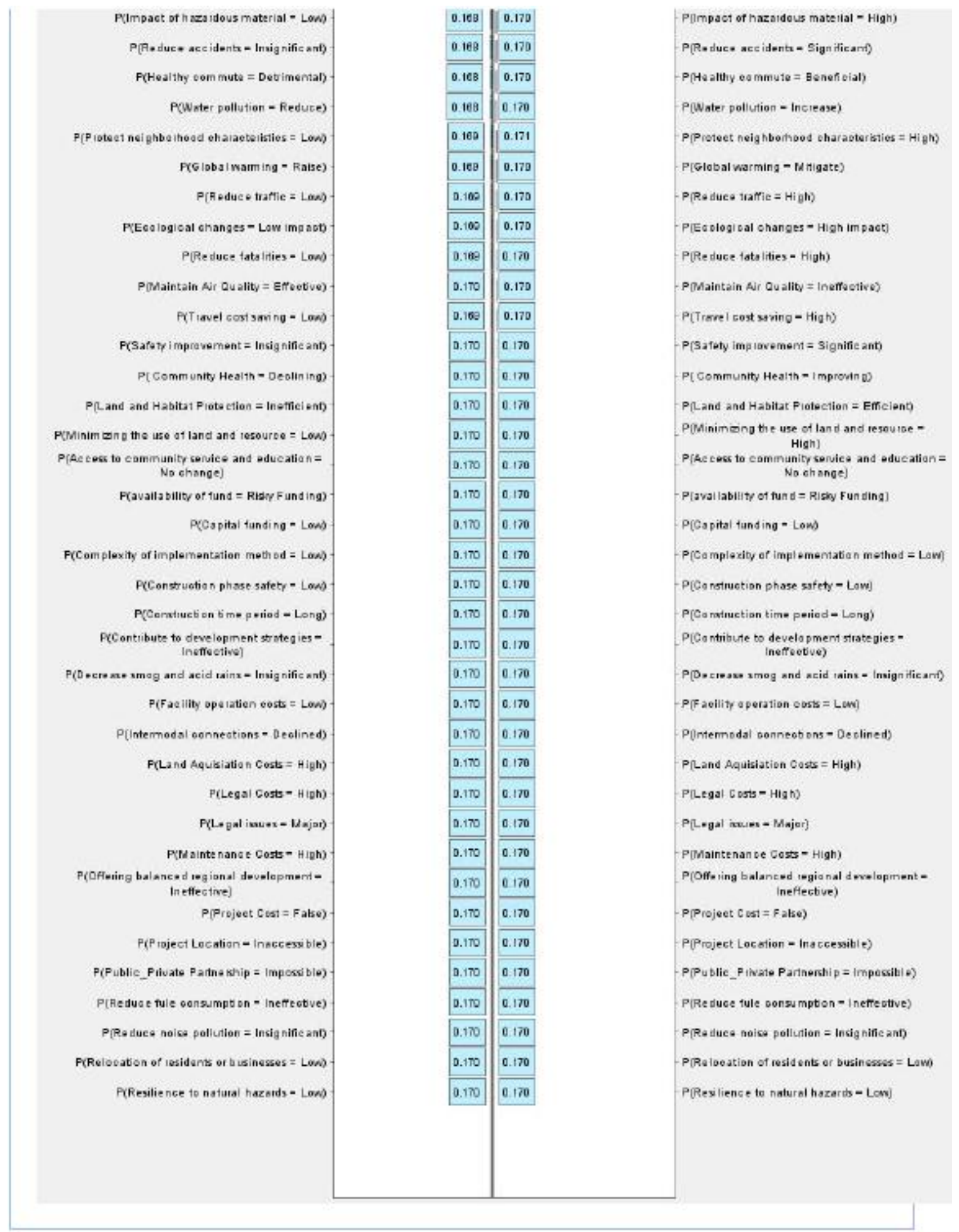


VITA

\section{NAHID VESALI MAHMOUD}

\section{EDUCATION \& EXPERIENCES}

Year

2016

2009

2006

2012-2014

2012-2016

2014
Details

Ph.D.

Florida International University

Department of Civil \& Environmental Engineering

Major area: Civil Engineering

Minor area: Construction Management

M.Sc.

Islamic Azad University

Science and Research Branch, Tehran, Iran

Department of Civil Engineering

Major area: Construction Management

B.Sc.

Iran University of Science and Technology, Department of Civil Engineering

Major area: Civil Engineering

Research Assistant

Florida International University

OHL School of Construction

Funded by FDOT

Teaching Assistant for following courses:

Structural Design I

Financial Management of Construction Organizations

Fundamentals of Construction Estimating

Construction Cost Analysis and Control

Decision and Risk Analysis in Construction

Developments in Construction Technology

Mentoring two guest students during their internship

\section{PUBLICATIONS}

1. Bayraktar, M. E., Arif, F., Ponder, D., Prozzi, J., \& Vesali Mahmoud, N. (2016). Opportunities for value extraction projects in highway rights-of-way and development of feasibility assessment tool. Journal of Construction Engineering and Management 
2. Vesali Mahmoud, N., Bayraktar, M.E., and Ozen, H. (2016). "A Conceptual Framework for Assessment of Project Alternatives in the Front-End Phase of Major Transportation Projects" In Construction Research Congress 2016 (pp. 1383-1392).

3. Vesali Mahmoud N., Bayraktar, M. E., \& Ozen, H. (2015). Method for Comparing Perceived Economic Impacts of Mega Transportation Project Alternatives. In International Interdisciplinary Business-Economics Advancement Conference ( $\mathrm{p}$. 352).

4. Vesali Mahmoud N., Bayraktar M. E. (2014), "Toward Sustainable Infrastructure: Comparing environmental impacts of potential sources of value extraction from highway right-of-way" Project Management Symposium 2014- June 9-10, 2014, College Park, MD, US 